\title{
IMPROVING REVISITATION IN LONG DOCUMENTS WITH TWO-LEVEL ARTIFICIAL-LANDMARK SCROLLBARS
}

\author{
A Thesis Submitted to the College of \\ Graduate and Postdoctoral Studies \\ In Partial Fulfillment of the Requirements \\ For the Degree of Master of Science \\ In the Department of Computer Science \\ University of Saskatchewan \\ Saskatoon, CANADA
}

By

Ehsan Sotoodeh Mollashahi

(C) Copyright Ehsan Sotoodeh Mollashahi, March, 2019. All Rights Reserved. 


\section{PERMISSION TO USE}

In presenting this thesis in partial fulfilment of the requirements for a Postgraduate degree from the University of Saskatchewan, I agree that the Libraries of this University may make it freely available for inspection. I further agree that permission for copying of this thesis in any manner, in whole or in part, for scholarly purposes may be granted by the professor or professors who supervised my thesis work or, in their absence, by the Head of the Department or the Dean of the College in which my thesis work was done. It is understood that any copying or publication or use of this thesis or parts thereof for financial gain shall not be allowed without my written permission. It is also understood that due recognition shall be given to me and to the University of Saskatchewan in any scholarly use which may be made of any material in my thesis.

Requests for permission to copy or to make other use of material in this thesis in whole or part should be addressed to:

Dean

College of Graduate and Postdoctoral Studies

University of Saskatchewan

116 Thorvaldson Building, 110 Science Place

Saskatoon, Saskatchewan S7N 5C9

Canada

Head of the Department of Computer Science

176 Thorvaldson Building

110 Science Place

University of Saskatchewan

Saskatoon, Saskatchewan S7N 5C9

Canada 


\begin{abstract}
Revisitation - returning to previously-visited locations in a document - is commonly done in the digital world. While linear navigation controls provide a spatial representation of the document and allow effective navigation in short documents, they are not effective in long documents, particularly for revisitation. Bookmarks, search and history dialogs, and "read wear" (visual marks left as the user interacts with the document) can all assist revisitation; however, for long documents all of these tools are limited in terms of effort, clutter, and interpretability. Inspired by visual cues such as coloured edges and "thumb indents" in hardcopy books, recent work has proposed artificial landmarks to help users build up natural spatial memory for the locations in a document; in long documents, however, this technique is also limited because of the number of pages each landmark represents. To address this problem, this thesis proposes a Double-Scrollbar design that uses two columns of artificial landmarks that can provide greater specificity for spatial memory and revisitation in long documents. We developed three versions of landmark-augmented DoubleScrollbar, using icons, letters, and digits as landmarks. To assess the performance and usability of the Double-Scrollbar design, two studies were conducted with 21 participants, each visiting and revisiting pages of a long document using each of the new designs, as well as a single-column design and a standard scrollbar. Results showed that two levels of icon landmarks were significantly better for assisting revisitation, and were preferred by participants. The two-level artificial-landmark scrollbar is a new way of improving revisitation in long documents by assisting the formation of more precise spatial memories about document locations.
\end{abstract}




\section{ACKNOWLEDGMENTS}

I would like to express my gratitude to my supervisor Carl Gutwin for his guidance and input throughout my graduate career at the University of Saskatchewan. I also wish to thank faculty and staff at the Department of Computer Science, and particularly to the students and staff in the HCI Lab for their help and their friendship. 


\section{CONTENTS}

Permission to USE

ABSTRACT

ACKNOWLEDGMENTS

CONTENTS

LIST OF TABLES

$\underline{\text { LIST OF FIGURES }} \quad \underline{\text { XI }}$

$\underline{\text { LIST OF ABBREVIATIONS }} \quad \underline{\text { XII }}$

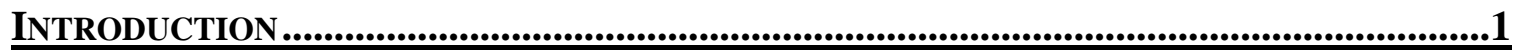

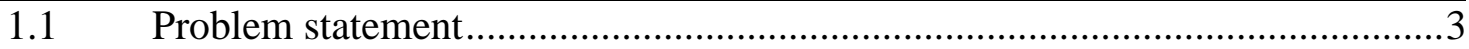

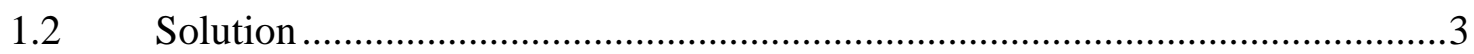

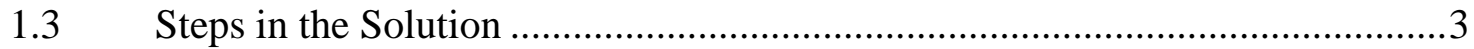

1.3.1 Implementation of two-level scrollbar augmented with landmarks .......... 3

1.3.2 Design variations in scrollbars with landmarks .................................... 5

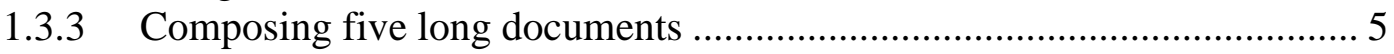

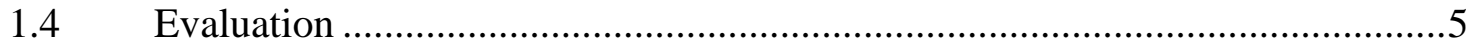

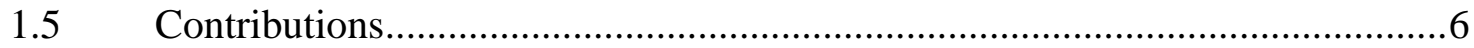

1.6 Thesis Outline ......................................................................................

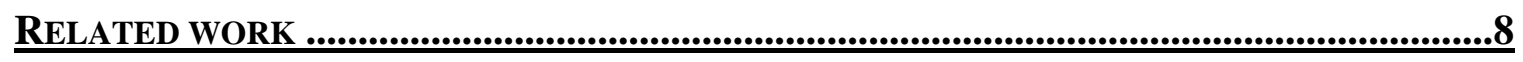

$2.1 \quad$ What is revisitation .......................................................................

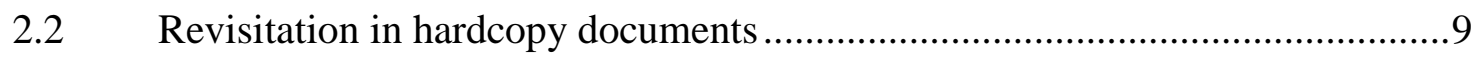

2.3 Revisitation in digital documents and interfaces ....................................11

2.3.1 Explicit revisitation techniques ...................................................... 11

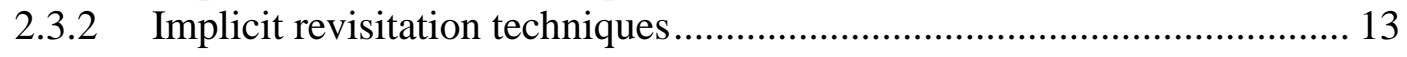

2.3.3 Landmarks and spatial memory ....................................................... 16

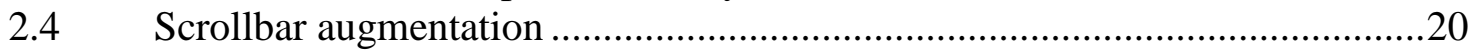

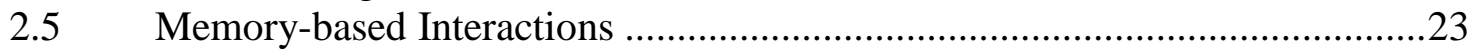

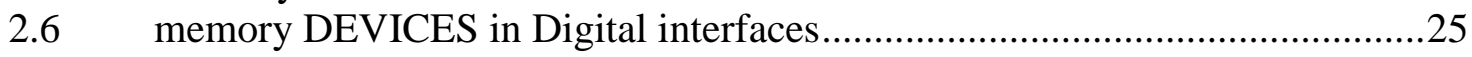

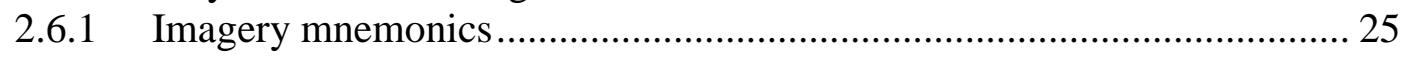

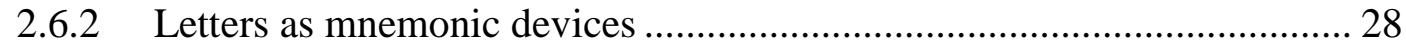


2.6.3 Chunking as a memory device

THE DESIGN OF A TWO-LEVEL ARTIFICIAL-LANDMARK SCROLLBAR ....................30

3.1 Landmarks for Linear Documents ............................................................... 30

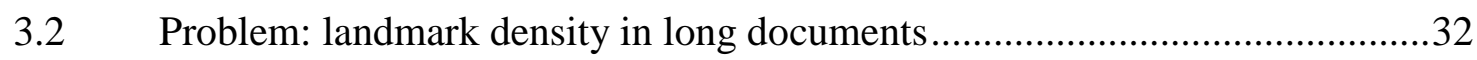

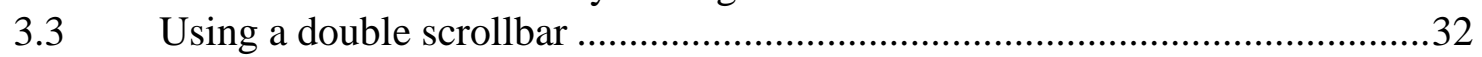

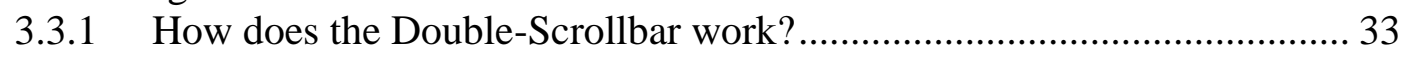

3.3.2 Questions to be answered about the feasibility of Double-Scrollbar........ 35

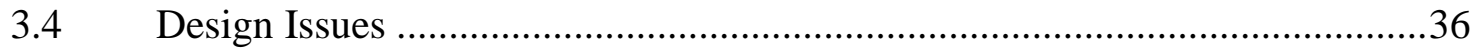

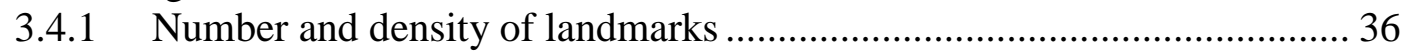

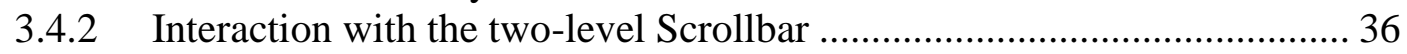

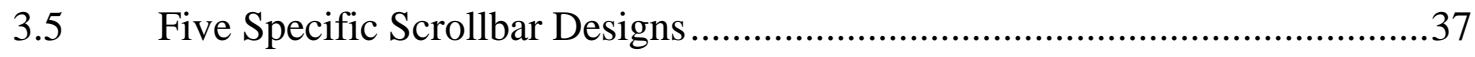

3.5.1 The standard scrollbar: Single-Scrollbar with no landmarks.................... 37

3.5.2 The Single-Icons design: Single-Scrollbar with icon landmarks.............. 38

3.5.3 The Double-Icons design: two scrollbars with icon landmarks................ 39

3.5.4 The Double-Letters design: two scrollbars with letter landmarks ............ 40

3.5.5 The Double-Digits design: two scrollbars with digit landmarks ............. 41

EVALUATION OF TWO-LEVEL LANDMARKED SCROLLBARS .....................................43

4.1 Study 1: Comparison of Standard scrollbar to landmark augmented scrollbars43

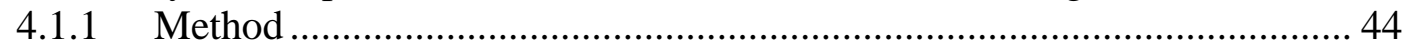

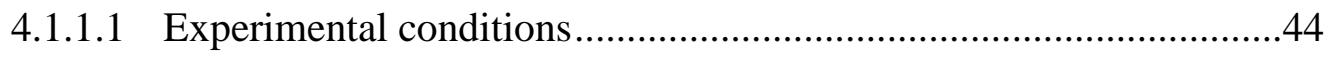

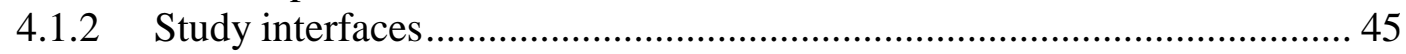

4.1.2.1 The Standard Scrollbar: Single-Scrollbar with No Landmarks.........45

4.1.2.2 Single-Icons: Single-Scrollbar with Icon Landmarks ......................46

4.1.2.3 Double-Icons: Two Scrollbars with Icon Landmarks ......................47

4.1.2.4 Double-Letters: Two Scrollbars with Letters as Landmarks..............48

4.1.2.5 The Double-Digits design: two scrollbars with digit landmarks.......49

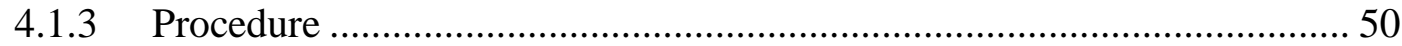

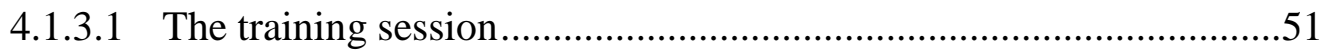

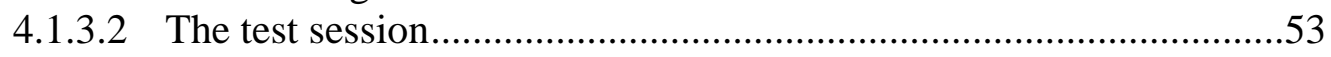

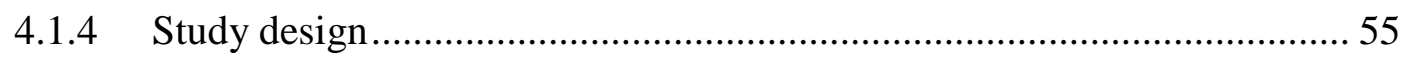

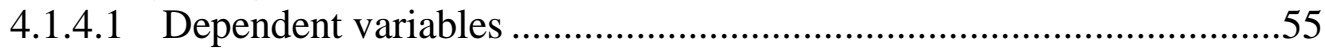

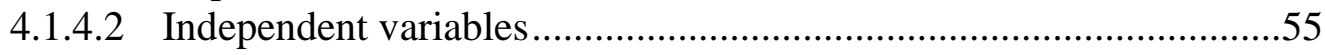

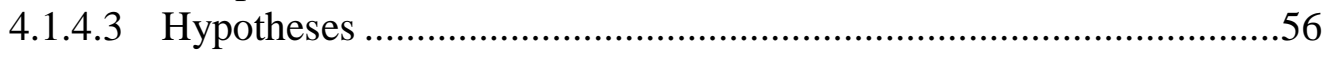

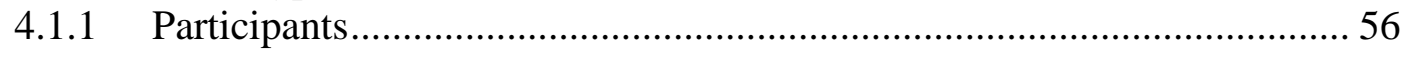

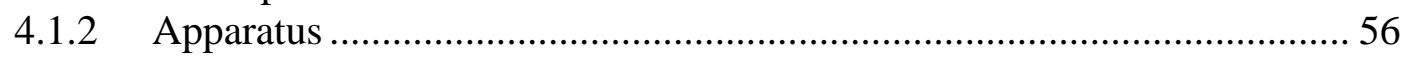

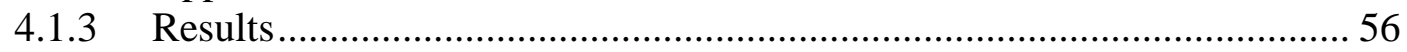

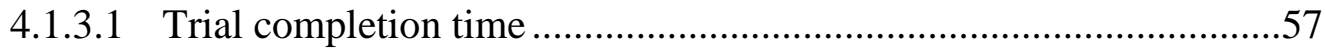

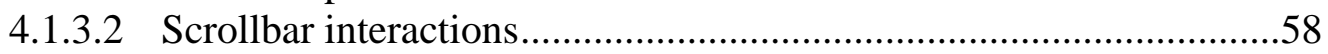

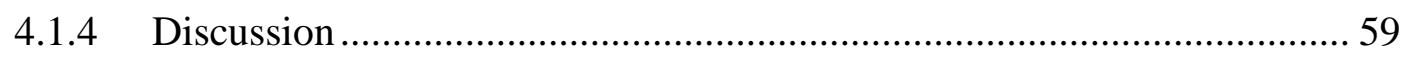

4.2 Study 2: landmark-based revisitation in large documents ............................59

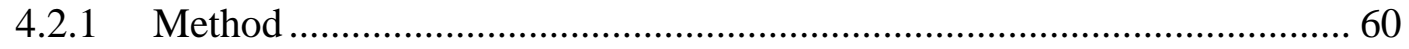

4.2.1.1 Experimental conditions, Study interfaces, Procedure, and Apparatus61 


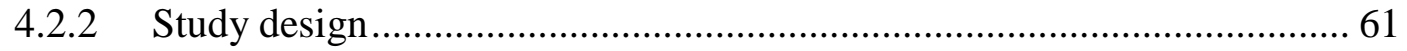

4.2.2.1 Hypotheses ..............................................................................62

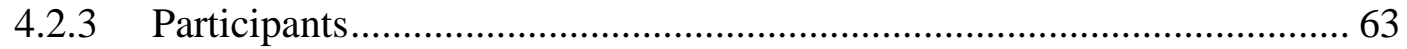

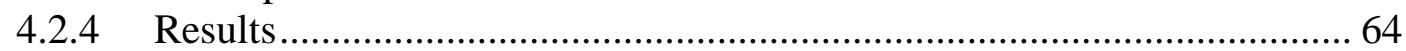

4.2.4.1 Trial Completion Time....................................................................64

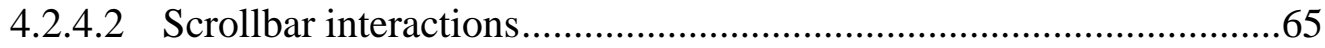

4.2.4.3 Time-out analysis ..........................................................................67

4.2.4.4 Analysis of the error distance in the first click..................................68

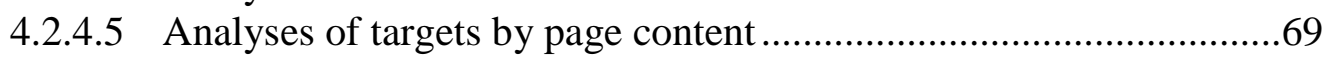

4.2.4.6 Analyses of the targets by proximity to landmark .............................71

4.2.4.7 Fit to the power law of practice.....................................................73

4.2.4.8 Subjective responses ....................................................................

4.2.4.9 Participants feedback .......................................................................76

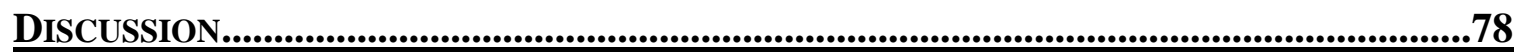

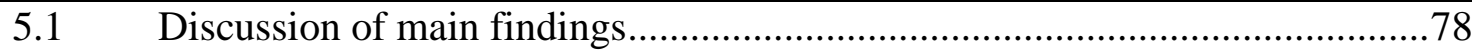

5.1.1 All landmarked scrollbars facilitated spatial revisitation.......................... 78

5.1.2 Two columns of landmarks are better than one ........................................ 79

5.1.3 Icons perform better as landmarks than letters and digits......................... 80

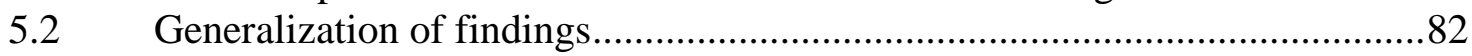

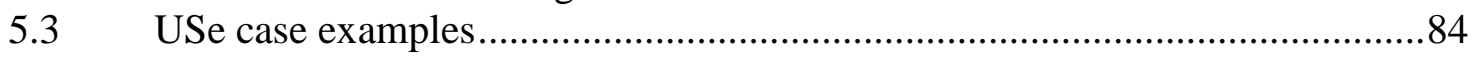

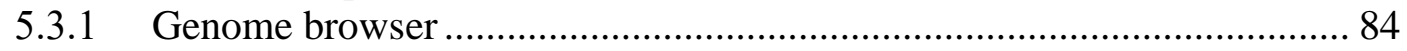

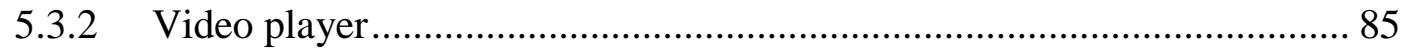

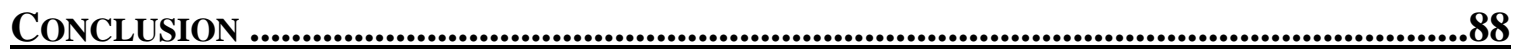

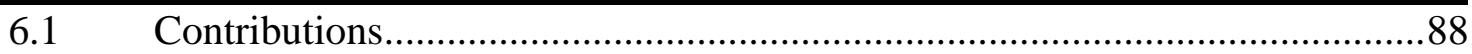

6.1.1 All Landmark-augmented scrollbars helped with revisitation.................... 88

6.1.2 The use of two columns of landmarks is feasible ..................................... 89

6.1.3 The Double-Icon design provided the best performance .......................... 89

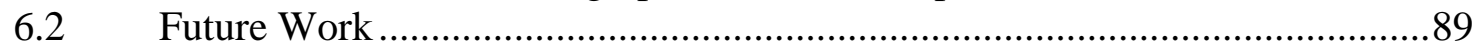

6.2.1 Different document content ................................................................ 89

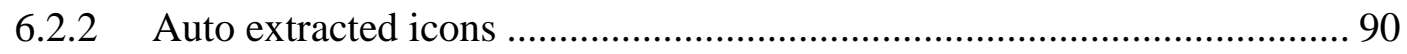

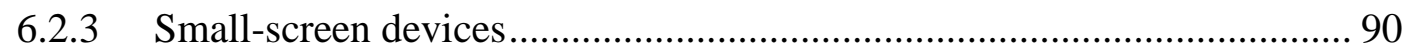

6.2.4 More than two levels of scrollbars ......................................................... 91

6.2.5 Combination with other techniques ......................................................... 92

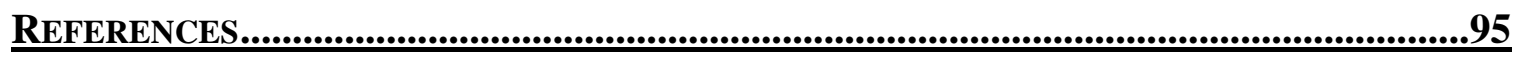

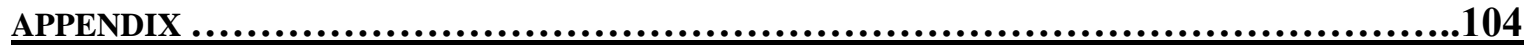




\section{LIST OF TABLES}

Table 4.1. Mean (s.d.) effort scores (0-10 scale, low to high). ...................................75

Table 4.2. Count of participants rating (0-10) of the ease of memorization for different interfaces.

Table 4.3. Count of participants' preferred interface on five aspects. .76 


\section{LIST OF FIGURES}

Figure 1.1 Double-Scrollbar augmented with icons as landmarks .4

Figure 2.1. A paper bookmark used to mark where reader ceased reading 10

Figure 2.2. Artificial landmarks on books. Left: patterns on page edges and thumb cuts showing the start of different sections in a dictionary. Right: colored page edges showing different sections in an anatomy text.

Figure 2.3. The keyword 'revisitation' has been repeated many times in the document..12

Figure 2.4. MS Word bookmarking menu 13

Figure 2.5. Implicit revisitation techniques. Left: Edit Wear and Read Wear (histograms in scrollbar show amount of user activity with that area of the document). Right: Footprints scrollbar, showing five previously-visited locations.

Figure 2.6. Recency list in Notepad++ 15

Figure 2.7. Using anchor points (grey squares in left figure) or background image (right figure) to assist formation of spatial memory 18

Figure 2.8. Hand as artificial landmarks: HandMark menu introduced by Uddin et al. to assist with revisitation of menu items in large tablets.

Figure 2.9. Uddin et al. scrollbar and slider augmentation. A: regular media player. B: media player with icon-augmented timeline. C: Media player with thumbnail-augmented timeline. D: Icon-augmented scrollbar. E: thumbnail-augmented scrollbar.

Figure 2.10. Code Thumbnail: Enhancement of revisitation by adding a miniature view of the document next to the scrollbar 
Figure 2.11. Left: yellow marks on scrollbar in Microsoft Visual Studio highlighting modified codes. Right: red marks in the same interface indicating location of complication errors.

Figure 2.12. Alpha Slider: augmenting a slider with letters as landmarks 23

Figure 2.13. Left: CommandMap, Middle: ListMap, Right: FastTap. Utilizing spatially stable menu icons to assist formation of spatial memory .25

Figure 2.14. Use of Mnemonic devices to assist memorization .26

Figure 2.15. Use of an image as a mnemonic device to help with item selection from a menu. 27

Figure 2.16. Using Order Mnemonics to remember the order of the Solar System planets29

Figure 3.1. Uddin et al.'s artificial-landmark augmented movie slider 31

Figure 3.2. Assigning one block of content to each icon on the coarse-grained scrollbar33

Figure 3.3. The arrangement of pages in each block 34

Figure 3.4. Study interface: Standard unadorned scrollbar 37

Figure 3.5. Study interface: Single-Scrollbar augmented with icons as landmark 38

Figure 3.6. Three type of landmarks placed on scrollbar(s). a: List of icons used for SingleScrollbar and Double-Icons interface. b: Letters used for Double-Digits interface. c: List of numbers used for Double-Digits

Figure 3.7. Study interface: Double-Scrollbar with icons as landmark .40

Figure 3.8. Study interface: Double-Scrollbar with letters as landmark .41

Figure 3.9. Study interface: Double-Scrollbar with digits as landmark .42 
Figure 4.1. The first and second studies environment and settings .44

Figure 4.2. Both studies run on a dual-monitor setting. Left monitor shows the target to find, and right monitor shows the custom document viewer

Figure 4.3. Study interface: Standard unadorned scrollbar. .46

Figure 4.4. Study interface: Single-Scrollbar augmented with icons as landmarks .47

Figure 4.5. Study interface: The Double-Scrollbar with icons as landmarks . .48

Figure 4.6. Study interface: Double-Scrollbar with letters as landmark . .49

Figure 4.7. Study interface: Double-Scrollbar with digits as landmarks .50

Figure 4.8. An example of the messages used to instruct participants during the study. .51

Figure 4.9. Training session process: A) shows the dual-monitor setting, with right monitor showing study interface. B) three target pages, C) maximum allowed time to visit each page, D) confirmation after each visit. Each colored rectangle represents one block of training (4 blocks for each interface) .52

Figure 4.10. Test session: A) shows the dual-monitor setting, with right monitor showing study interfaces and left monitor study stimuli; B) three screenshots of target pages shown as stimuli on left monitor; C) target pages in the document shown on right monitor; D) maximum allowed time to visit each page; E) confirmation after each revisit. Each colored rectangle shows one block of the test session (4 blocks for each interface) .53

Figure 4.11. The feedback message for a successful revisitation. 54

Figure 4.12. The feedback message for an unsuccessful revisitation. .54

Figure 4.13. Trial completion time ( \pm s.e.) by block. .57

Figure 4.14. Total scrollbar interactions by block. .58 
Figure 4.15. On landmark (a. and c.) and near landmark (b. and d.) in Double-Scrollbar and SingleScrollbar designs

Figure 4.16. Examples of pages used as targets....................................................63

Figure 4.17. Mean completion time ( \pm s.e.) by condition and block.............................64

Figure 4.18. Scrollbar interaction ( \pm s.e.) by condition and block. ..............................65

Figure 4.19. Scrollbar interactions ( \pm s.e.) by activity ..........................................66

Figure 4.20. Mean number of time-out ( \pm s.e.) by block ........................................67

Figure 4.21. Mean distances ( \pm s.e.) of scrollbar interactions. ...................................68

Figure 4.22. Analyses of mean completion time based on page content. ......................69

Figure 4.23. Analyses of the number of interactions based on page content...................70

Figure 4.24. On landmark - near landmark in Single-Scrollbar and Double-Scrollbar designs

Figure 4.25. Analyses of time by landmark proximity. ........................................72

Figure 4.26. Analyses of the number of interactions by landmark proximity. ...............73

Figure 4.27. Regression analysis of task completion time across blocks for all landmark

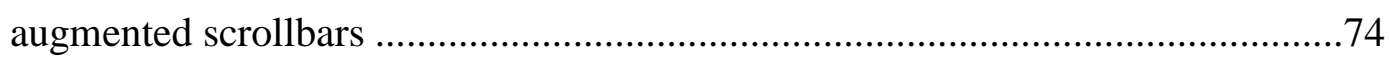

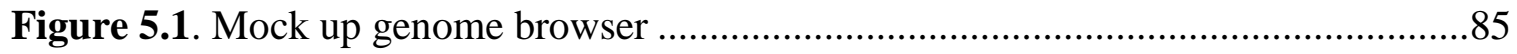

Figure 5.2. Previews of a twenty-minutes video on YouTube timeline .......................86

Figure 5.3. Mock up video player with two levels of scrollbar augmented with icons ....87 
Figure 6.1. A: Unadorned bookmarks on the scrollbar. B: Icon-augmented bookmarks on the scrollbar...

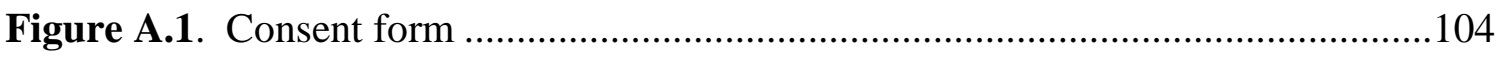

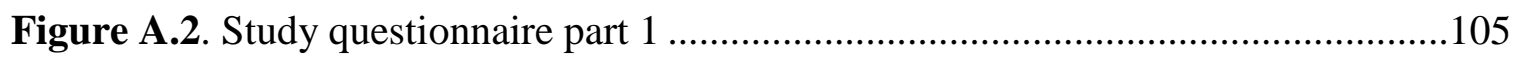

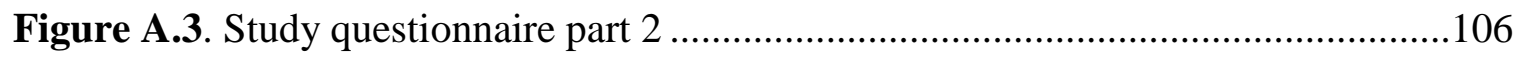

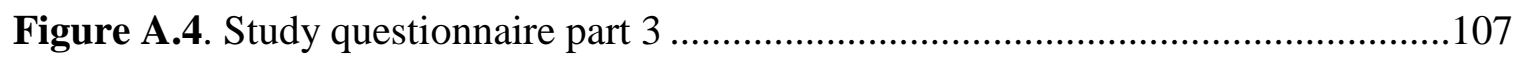

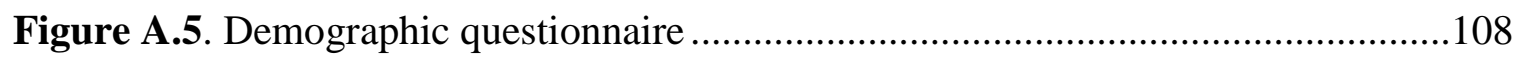




\section{LIST OF ABBREVIATIONS}

2D

GUI

HCI

OS

UI
Two Dimensional

Graphical User Interface

Human-Computer Interaction

Operating System

User Interface 


\section{CHAPTER 1}

\section{INTRODUCTION $^{1}$}

Since the emergence of computers, viewing and manipulating different types of digital content has become an essential function of computational work. The increased power of computers over the course of time has led to a widespread use of them to open, view, and review different types of long documents, such as long audio podcasts, multi-hundred-page text files, or long genome sequences. Digitized documents provide ease of access, storage, duplication, and sharing to users. However, they have some downsides, such as poor support for revisitation (i.e. finding a location in a document that the users have been to before). For example, digital documents lack the physical cues that physical books normally have (e.g. indentations or colored page edges) that help readers with navigation tasks. Due to the importance of revisitation in digital contexts, this thesis introduces a new interaction method that takes advantage of artificial landmarks as added cues to help people revisit locations in a document.

Revisitation is the process of navigating to a location in a document or interface that has been visited in the past. People tend to repeat a task over the course of time for different purposes. For example, they visit their favorite websites on the internet on a daily basis, open their favorite apps on an hourly basis, call certain phone numbers repeatedly, or bookmark or highlight parts of a book or a document to revisit them faster in future. Previous research suggests that revisitation is commonplace. In 1993, Greenberg reported that 57\% of telephone numbers are redialed [35], and Lee found that $75 \%$ of Unix commands are entered frequently [53]. Cockburn and McKenzie also found that $81 \%$ of web pages have been previously visited by the users [20]. As another example, Alexander et al. analyzed log activity of 14 participants who used Microsoft Word and Adobe

\footnotetext{
${ }^{1}$ Portions of this thesis appeared in the following publication:

Ehsan Sotoodeh Mollashahi, Md. Sami Uddin, and Carl Gutwin. 2018. Improving revisitation in long documents with two-level artificial-landmark scrollbars. In Proceedings of the 2018 International Conference on Advanced Visual Interfaces (AVI '18). ACM, New York, NY, USA, Article 30, 9 pages. DOI: https://doi.org/10.1145/3206505.3206554
} 
Reader for 120 days, and realized that of the 2700 documents that were navigated, $29 \%$ had been revisited at least once [4].

Even though there are different techniques to assist with revisitation, none of them works very well in long documents. Previous techniques can be put into two main groups: explicit and implicit revisitation tools.

Explicit revisitation tools require users' active engagement. For example, bookmarking is a common tool in many interfaces to aid revisitation. However, many users do not add bookmarks because of the effort: thinking about when to place a bookmark (and going through menus to get to the bookmark menu and then typing a label for the bookmark) can be a burden to users as it interrupts their train of thought.

Implicit revisitation tools are decided upon by the system, rather than users. Read Wear is an example of an implicit revisitation device: this technique helps with revisitation by placing marks on the screen (e.g. the scrollbar) based on user behavior. The advantage of this method is that it places marks automatically as users read the document (e.g. the time users spends on a specific page), which does not interrupt users' train of thought. However, implicit techniques can sometimes miscalculate the areas of interest (e.g., if the user is interrupted by another action such as a phone call).

A different type of implicit support for revisitation is the idea of artificial landmarks - that is, placing spatially stable landmarks on a scrollbar, movie slider, or background of a menu. Landmarks assist users in building spatial memory of locations in documents, and can be effective in supporting revisitation (e.g. Uddin et al. showed that icon landmarks can benefit revisitaition $[88,90])$. However, current designs for augmenting navigation controls with artificial landmarks show shortcomings when it comes to revisitation in long documents, due to the limited number of icons that can be accommodated into widgets such as a scrollbar.

In this thesis, we designed an augmented interface with artificial landmarks on a two-level scrollbar to accommodate much longer documents than what have been considered previously. 
The artificial landmark approach removes the effort of bookmarking, and the two-level design allows much finer granularity in the mapping between locations and landmarks.

\subsection{PROBLEM STATEMENT}

Even though revisitation in digital interfaces is a common task, current revisitation techniques are not effective in long documents. In particular, artificial landmark techniques are not well suited to long documents because the mapping between locations and landmarks is too coarse.

\subsection{SOLUTION}

The solution to the problem stated above is to add another level of landmarks to previous designs, to allow a more precise mapping between document locations and landmarks. Two levels of landmarks means that each location is associated with two landmarks - one coarse-grained and one fine-grained. The goal of the two-level design is to improve precision through the added specificity of landmarks. This potential advantage has the cost, however, of users needing to remember two landmarks for each location.

\subsection{STEPS IN THE SOLUTION}

Our solution has been implemented in three parts as follows:

\subsubsection{Implementation of two-level scrollbar augmented with landmarks}

One of our underlying assumptions in this study was that current Single-Scrollbar artificiallandmark designs do not provide fine-grained navigation for long documents. For instance, if a document is 30 pages long, and the scrollbar is 900 pixels high, each 10-pixel movement of the scrollbar handle changes the location in the document by a third of a page. Using the same 
interface, if the document is 900 pages long, each 10-pixel movement of the handle translates into a 10-page movement, which makes precise selection of a specific page very difficult.

The other problem with previous designs is that when the document is short (e.g. 30 pages) and there are 30 icons on the scrollbar, each icon will be mapped to one page. This design makes the formation of memory much easier. In a 900-page document with the same interface, each icon is mapped to 30 pages, which makes it significantly harder to form a connection. Therefore, in our design, we divided the content of the document by 30 and called each portion a block no matter how long the document was. Then, we represented each block of the content with an icon on the left scrollbar (coarse-grained scrollbar) and then divided each block by 30, mapping these subblocks to 30 icons on the right scrollbar. In this design, if users want to open page 68 of a 900page document, they would have to click on the second icon of the coarse-grained scrollbar and then the eighth icon on the fine-grained scrollbar (see Figure 1.1).
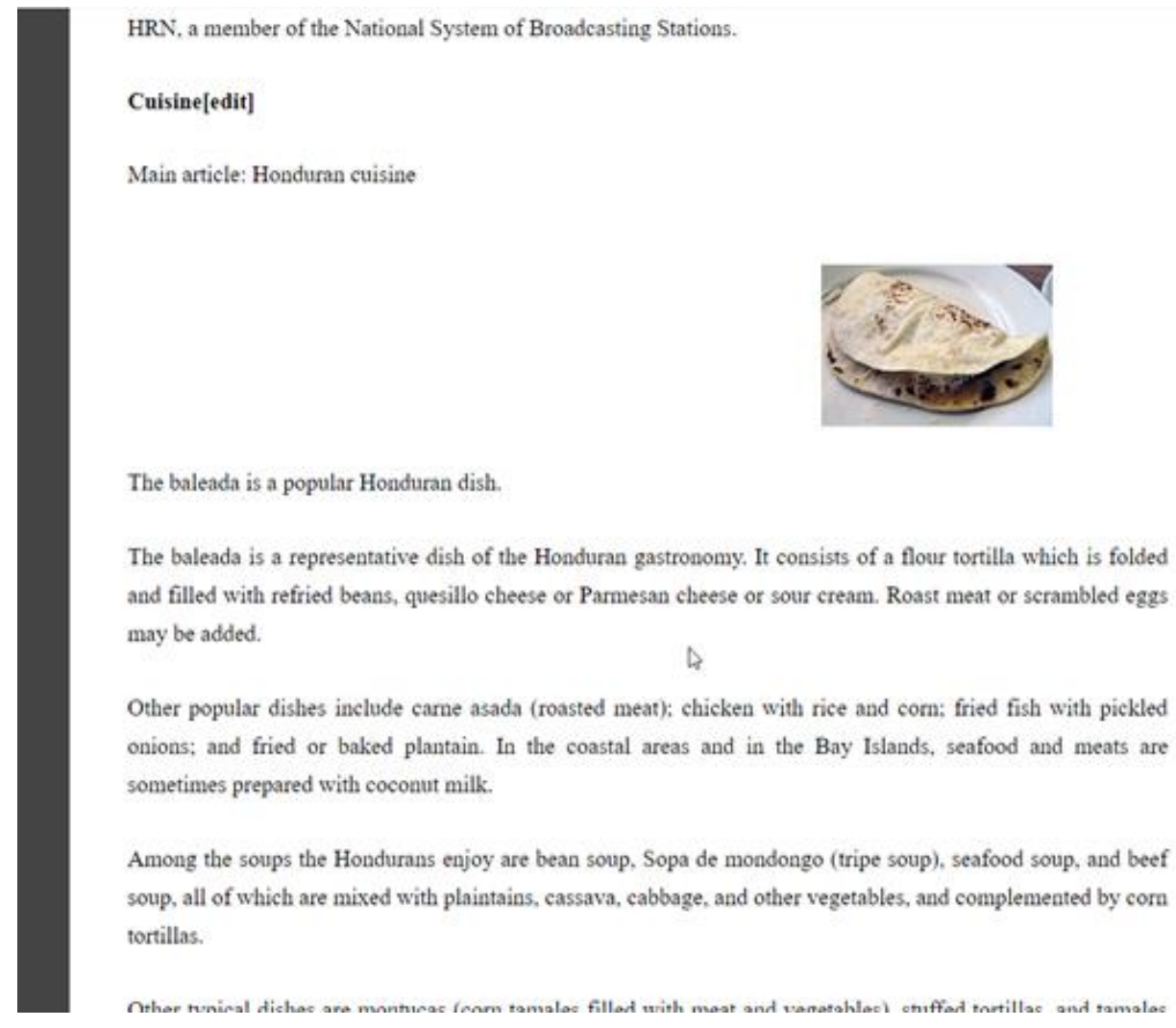

Among the soups the Hondurans enjoy are bean soup, Sopa de mondongo (tripe soup), seafood soup, and beef soup, all of which are mixed with plaintains, cassava, cabbage, and other vegetables, and complemented by corn tortillas.

Figure 1.1 Double-Scrollbar augmented with icons as landmarks 


\subsubsection{Design variations in scrollbars with landmarks}

We augmented our two-level scrollbar design with 30 landmarks (27 pixels in width, 27 pixels in height, monochrome) added vertically to each scrollbar. Because of the human vision limitations, we used only 30 landmarks in each scrollbar to ensure that landmarks were easily distinguishable and understandable. We developed three forms of the augmented scrollbar, using three kinds of landmarks: letters, digits, and icons. These provided different forms of landmarks and allowed us to compare the efficiency of different designs.

\subsubsection{Composing five long documents}

For our two studies, we composed numerous long documents $(900+$ pages $)$ by putting together random excerpts from Wikipedia articles on a variety of topics. Contents included text, titles, and images of different sizes and different alignments.

\subsection{EVALUATION}

To measure the usefulness of our two-level scrollbar interfaces, we ran a small preliminary study and a full study to compare them against a standard (unaugmented) scrollbar and the single-level icon scrollbar seen in previous work [82]. Our goal was to answer the following questions:

- Does the two-level scrollbar outperform the standard scrollbar and the icon-augmented Single-Scrollbar?

- Is the two-level scrollbar easy to use and learn?

- How well does the single-level design work in long documents?

- Among icons, digits, and letters, which type of landmark works best? 
To answer these questions, we carried out two studies (one with five interfaces, and one with four) in which participants were asked to visit and revisit several locations in long documents, using each of the interfaces (with different documents for each interface). Users to do a visit were shown some pages of the document, and for a revisit they had to recall the location of given pages and browse to find them. The preliminary study clearly showed that the unaugmented scrollbar was significantly slower for revisitation tasks than all of the artificial-landmark interfaces. Therefore, the second study removed the standard scrollbar and compared the augmented interfaces in more detail. Results from both studies suggest that two-level icon scrollbars are faster than single-level scrollbars, and are not substantially more difficult to learn (see 4.2.4.7) (however, two-level scrollbars with letters and digits were not faster). Comparing users' activity logs suggests that they were able to make effective use of the finer granularity of the two-level icon scrollbar, and that icons facilitated the formation of spatial memory better than digits or letters. Two-level scrollbars were also preferred by users.

\subsection{CONTRIBUTIONS}

The primary contributions presented in this thesis are the following:

First, even though current scrollbars as linear navigation controls in document viewers provide a spatial representation of the document, they show a clear shortcoming when it comes to supporting spatial memory. Therefore, they are insufficient for revisitation in long documents. All of the landmark-augmented interfaces were significantly faster, less prone to error, and preferred more by users compared to an unaugmented scrollbar. This provides additional evidence that artificial landmarks can help to develop spatial memory and can improve revisitation performance in long documents.

Second, two-level icon scrollbars had significantly lower numbers of interactions compared to single-level icon scrollbars, but were not significantly faster overall. 
Third, by comparing icons, letters, and digits as landmarks in these studies, we showed that icons were better in facilitating the spatial memory formation, and digits were the worst. Users' comments suggested that it was easier to form a story that connected the content of the page to the corresponding combination of the icons on the scrollbars.

\subsection{THESIS OUTLINE}

The content of this thesis is organized in five chapters. In Chapter Two, we present a literature review to form a foundation for the research. We first introduce current techniques for improving revisitation in digital and physical documents. Then, we explore the use of artificial landmarks in interfaces. Third, we discuss different scrollbar augmentation methods. Fourth, we review the definition of spatial memory and its application in digital interfaces. Finally, we describe mnemonics and their role in digital interfaces. In Chapter 3, we will introduce the study interfaces and provide details on their implementation. In Chapter 4, we explain the experimental setup, and discuss the results of both studies. In Chapter 5, we discuss the results and provide explanations for our findings. Finally, in Chapter 6, we summarize our findings and primary contributions, and present future directions that can be explored to complement and add to this research topic. 


\section{CHAPTER 2}

\section{RELATED WORK}

Augmenting scrollbars with landmarks to address the problem of revisitation in long documents is based on six areas of research. This literature review gives us insight about current revisitation techniques, challenges involved with each of them, and possible solutions. In the first section below we will introduce revisitation and explain why it is important to address it. In the second and third sections, we will introduce different revisitation techniques in hardcopy and digital documents that have been used or investigated. We will also discuss the shortcomings related to each of these methods. Knowledge acquired from the first section as well as drawbacks of current revisitation methods (discussed in the third section) motivated us to develop the Double-Scrollbar design as a solution to the existing limitations. Since we took advantage of augmented scrollbars with landmarks, the fourth section will explore related work on scrollbar augmentation. We will present different techniques which provided information or ease of access to desired location in the document using scrollbar augmentation. Exploring different methods of scrollbar augmentation will assist us with designing a more intuitive and efficient interaction method. In the fifth section, we will discuss memory-based interaction methods; therein, we explain why memory-based interactions are crucial and what techniques have been developed to take advantage of it. Finally, in the last section, we will examine different types of mnemonics as memory tools. Insight gained in the last two sections will help us augment our scrollbars with landmarks that are easier to remember and distinguish.

\subsection{WHAT IS REVISITATION}

Revisitation is a type of user interaction with documents or interfaces that involves navigation to locations that have been visited in the past, in order to re-access information or re-select tools. Revisitation in digital contexts involves a variety of activities, including returning to a particular website [2,8,18,25,42,95], re-accessing or modifying information in a document [82], switching 
between opened windows or tabs in a browser [50], scrubbing the timeline of video player to find previously-watched scenes [49], or re-selecting a menu item [83].

An abundance of research demonstrates that revisitation is a common task in digital interfaces. For instance, in the context of web revisitation, different studies reported that a significant number of accessed webpages were previously visited in past; the ratio of revisitation to new visits varies from one study to another, ranging from $58 \%$ to $81 \%$ [20,86]. Also, in the area of map revisitation, Zhao et al. conducted a survey on map browsing habits of 30 users and stated that almost half of the participants frequently searched their maps for a previously visited locations [89]. As another example, Alexander et al. examined revisitation in files by logging the Microsoft Word and Adobe Reader activities of 14 participants for 120 days. They showed that out of the 2700 documents that were opened, $29 \%$ of them had been revisited at least once [4].

\subsection{REVISITATION IN HARDCOPY DOCUMENTS}

Historical evidence suggests that revisitation tools are almost as old as books. For example, physical bookmarks are a common revisitation aid - using pieces of paper, leather, or fabric to keep a places in a book to ensure an easier return to that spot [78]. The form, type, and material used for bookmarks has gone through many changes but they still serve the same purpose of helping readers return to where they left off (see Figure 2.1). 


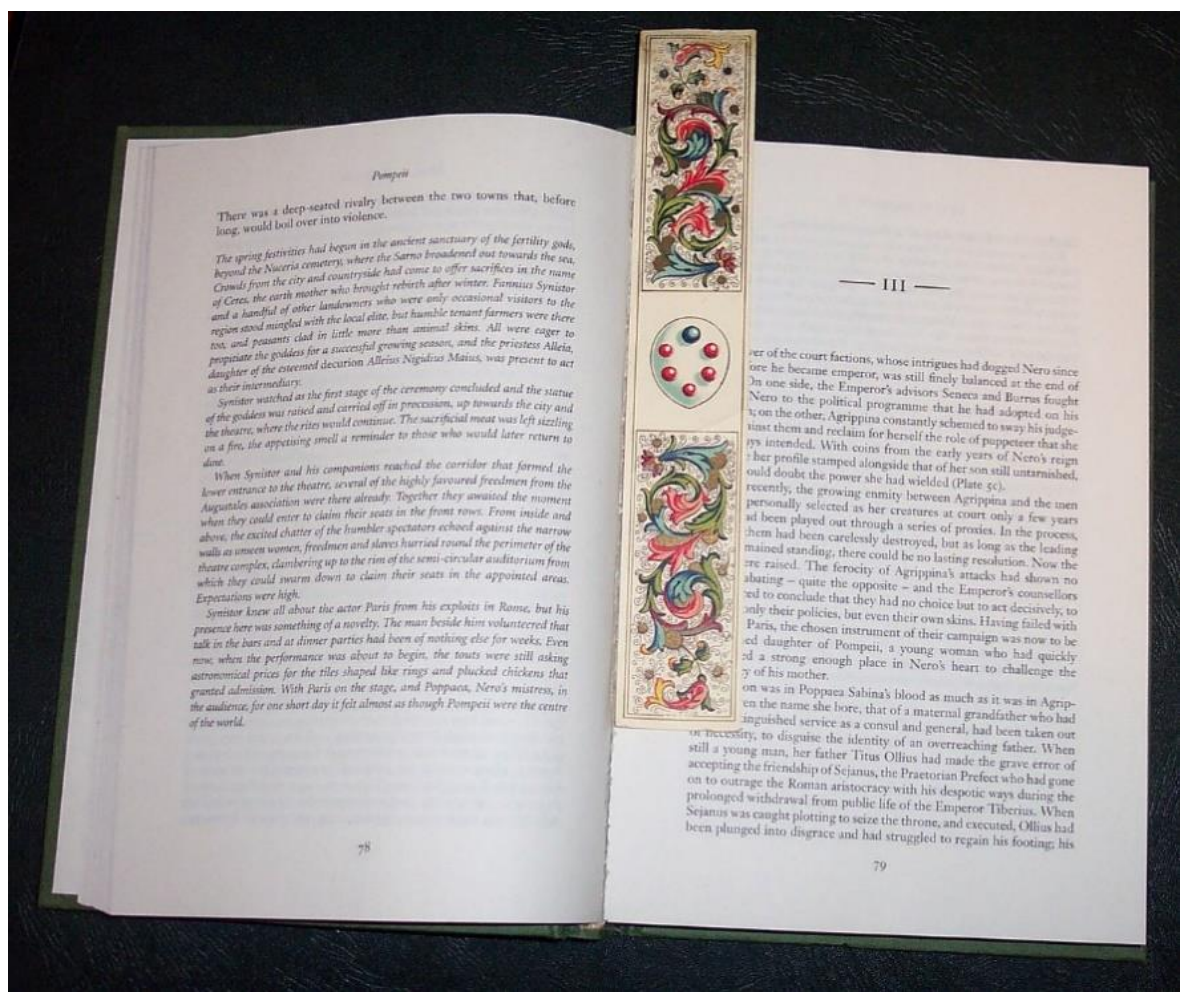

Figure 2.1. A paper bookmark used to mark where reader ceased reading

Over time, other techniques were developed to improve navigation in reference books (particularly long documents). For example, "thumb cuts" or indentations in the pages of many dictionaries, reference books, and religious books offer faster access to the start of a letter or a section of that book (Figure 2.2, left), and give a reference frame for remembering locations. Coloured edges in some reference books (Figure 2.2, right) also serve as visual cues to facilitate the re-finding of a certain part of a document. 


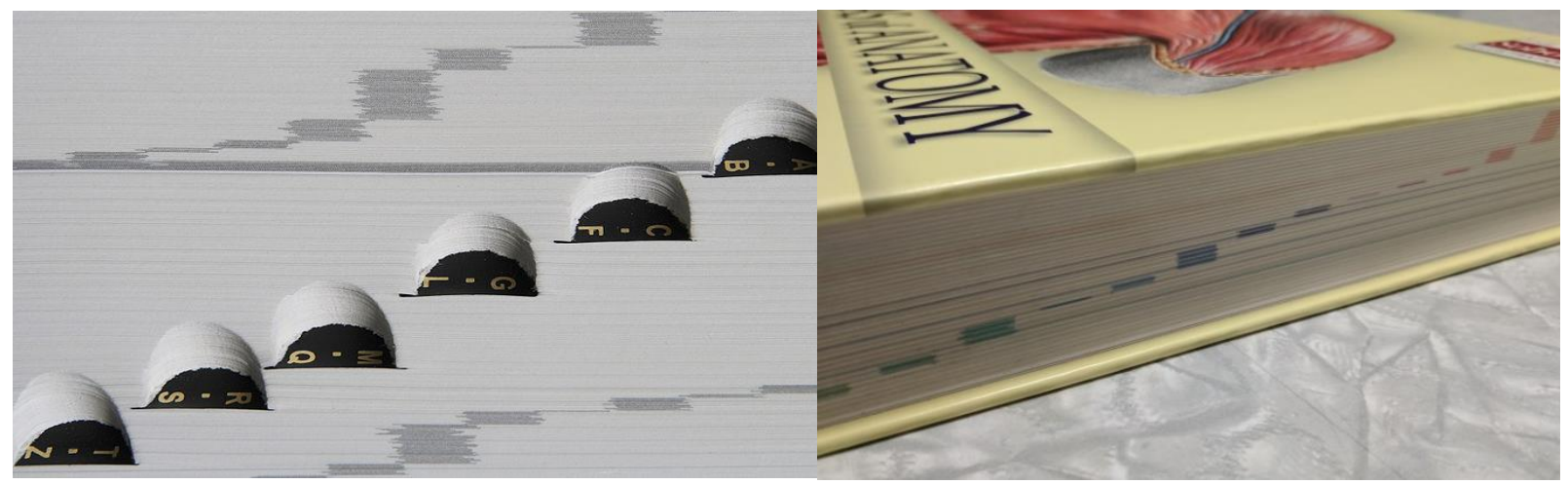

Figure 2.2. Artificial landmarks on books. Left: patterns on page edges and thumb cuts showing the start of different sections in a dictionary. Right: colored page edges showing different sections in an anatomy text.

\subsection{REVISITATION IN DIGITAL DOCUMENTS AND INTERFACES}

Tools developed to support revisitation in long documents can be categorized into two main groups: explicit and implicit methods.

\subsubsection{Explicit revisitation techniques}

Explicit revisitation methods are interaction methods that require users' active engagement in revisiting areas of interest. In the following we will introduce tools that can be used as navigation tools or revisitation tools (if the user has visited that location in past). Search bars are one of the most popular explicit revisitation and navigation tools and are employed in many file browsers, internet browsers, websites, and document viewers. These widgets facilitate the revisitation process by highlighting searched keywords and taking users to the respective spot in the document. However, when the keywords users are looking for are repeated many times in the document, a search process will turn into a cumbersome process of visiting many locations in the document and examining each to see if that is the right one (see Figure 2.3). Moreover, the necessity of knowing the exact keyword is reported to be a major usability issue $[8,56,62,84]$, making the 
search technique less efficient in long documents. Also, search only works in text files and has no use in audio and video files.

users (as mentioned by Alexander's et a Find
used bookmarking strategy introduc revisitation
Word)[2]. Laakso 's bookmarking and
wear both have the advantage of represe
or bookmark on the scrollbar. This del. Previous
immediate access to the bookmark or read-wear (revisitation
marks) on the scrollbar. Though, this technique showed to be
more effective with few number of bookmarks.
Visualizing revisitation marks on the scrollbar seems to be
an efficient way of representing them in terms of speed, the
number of errors and user preference [2]. However
increasing the number of marks can decline their
effectiveness by increasing the clutterness of visualization
and increasing the time to make the decision to choose the
right revisitation mark as indicated by Hick's Law. To
evaluate how an increase in the number of revisitation marks
can affect the performance of the Laakso's strategy, we ran
a mini study. Our mini-study showed that increasing number
of the similar looking revisitation marks can increase the
time and number of errors people make when they try to find
the right revisitation mark. We believe that similar looking
squares used to represent the revisitation marks do not
provide enough features that distinguish them from the rest

Figure 2.3. The keyword 'revisitation' has been repeated many times in the document

Bookmarking is another common revisitation tool in many document viewers and web browsers. Similar to classic physical bookmarks, bookmark tools in digital interfaces allow users to place flags in areas of interest within or between documents.

However, there are some major problems regarding the use of bookmarks within documents. First, users have to identify the pages of interest for revisitation in future, which might not be clear beforehand. Second, placing and opening a bookmark requires many steps. Users usually need to go through a hierarchy of menus to find the bookmark menu, come up with a label for a bookmark, type it in to the dialog, and press a "save" button. To open a bookmark they have to go through the same menu hierarchy, do a visual search to find the right bookmark from a list of available bookmarks and finally select it (see Figure 2.4). 


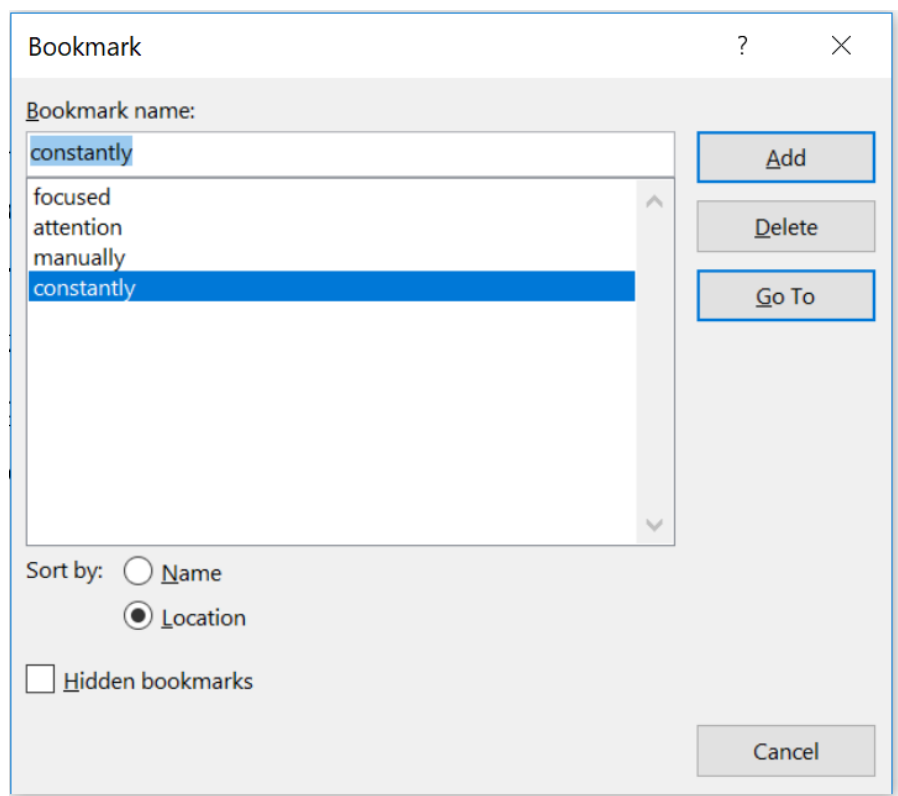

Figure 2.4. MS Word bookmarking menu

Pitkow reported that this long process can discourage users from using this technique and can lead to suboptimal strategies, such as scrolling and relying on visual search [26]. As shown in the usage patterns reported by Alexander et al. regarding navigation in Microsoft Word and Adobe Reader, the overhead cost of bookmarking can discourage use of this feature in the first place [4].

To remove some of the steps involved with placing and invoking bookmarks, Laakso et al. proposed visualizing bookmarks on the scrollbar [52]. Even though this design provides ease of access to bookmarks, it fails to address another important issue about bookmarking tools. Reading demands a focused attention, while manually placing bookmarks requires thinking whether or not a particular part of the document is worthy of being bookmarked, which constantly interrupts readers' train of thought and increases their cognitive and operational load $[1,41,42]$.

\subsubsection{Implicit revisitation techniques}

The limitations of explicit revisitation tools encouraged many researchers to look for alternative methods that do not need active engagement to capture locations of interest in a document. Placing automatic bookmarks or "Read Wear" based on the user's behavior in reading a document is one 
of the areas they explored. In 1992, Hill et al. designed "edit wear and read wear" techniques that showed histograms of a user's visit and edit history on the scrollbar (see Figure 2.5: Left) [38]. This approach later inspired Alexander et al. and Yu et al. to use the time readers spent on a certain part of the document to place revisitation marks on the scrollbar $[4,88]$. In their designs, a visit was defined as a pause for a certain number of seconds on a part of document, and was marked as a colored cue on the scrollbar based on the recency of revisitation or the number of revisitations. Skopik et al. introduced a similar idea called Visit Wear by augmenting fisheye views ${ }^{2}$ with visual effects to provide more support for revisitation. All of these designs aim to change the task of revisitation to a combination of visual search memorization [76].

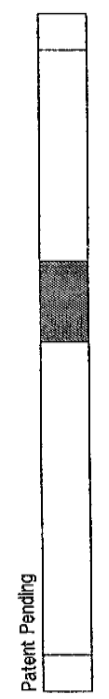

(a)

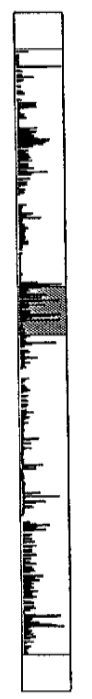

(b)

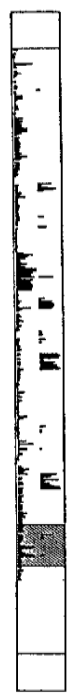

(c)

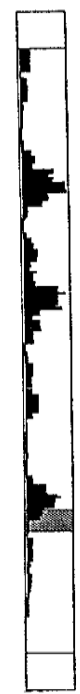

(d)

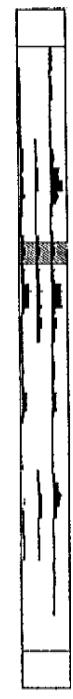

(e)

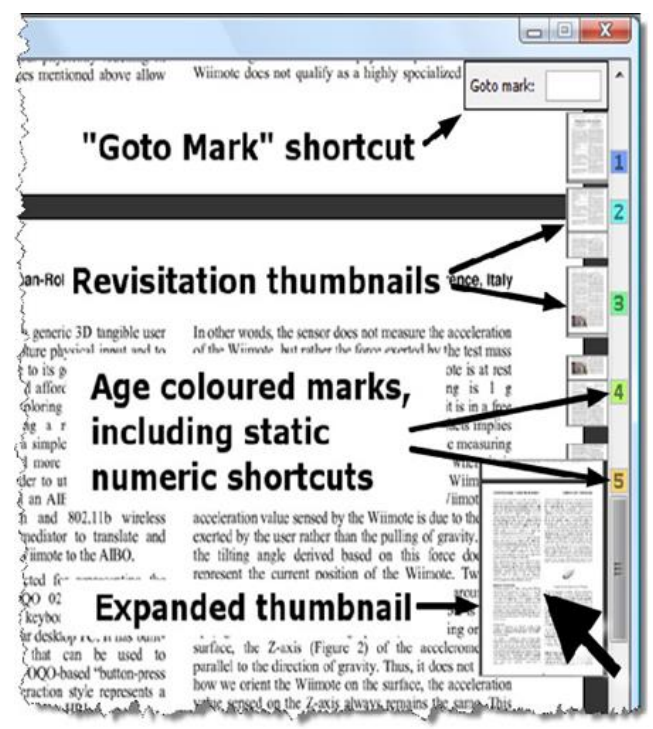

Figure 2.5. Implicit revisitation techniques. Left: Edit Wear and Read Wear (histograms in scrollbar show amount of user activity with that area of the document). Right: Footprints scrollbar, showing five previously-visited locations.

However, sometimes this design fails to capture the real intention of users and therefore fails to serve its purpose. As an example, users might spend more time on a specific page of the document

\footnotetext{
${ }^{2}$ Fisheye view is a visual distortion technique that resembles a fisheye paranematic view, to provide an overview of bigger part of the dataset at same time with a magnified view of some parts [14].
} 
for different reasons, including a distracting phone call or a short discussion with a colleague, which places misleading marks of high importance in the document.

A history list records users' browsing history to enable faster revisitation of a page. However, since users visit many pages daily, history lists become long and require a costly visual search to find an item.

Recency lists are another technique introduced to aid revisitation by providing users with a subset of recently visited files or commands. For instance, Figure 2.6 shows a list of recently opened files in Notepad++. The underlying assumption about the recency list is that a clear majority of revisitations usually happen for the last viewed items. For example, Tauscher et al. showed that $81 \%$ of webpage revisitations can be addressed using recency lists [95]. However, the problem with recency list is that when the number of recently viewed items on the list is large, a visual search to find the desired option will be cumbersome. Furthermore, if the item users are looking for is not on the list, it adds another step to the search process which can be costly in terms of users' time and satisfaction. Another major problem with recency list is the lack of support for document revisitation - that is, the parts of a document that are to be revisited are not necessarily the most recent parts [4].

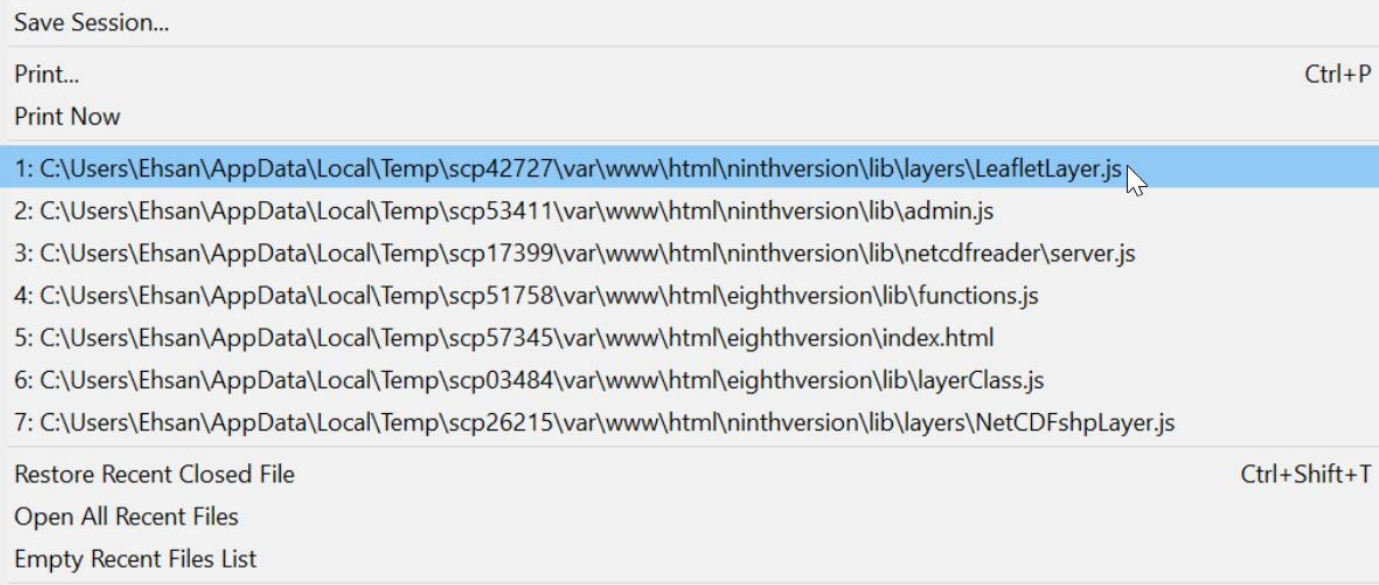

Figure 2.6. Recency list in Notepad++ 
The Back/Forward buttons and visualization of visited hyperlinks (e.g., using a different colour) are other means of supporting revisitation, mostly in web browsers. However, both techniques are also limited. For instance, using the Back/Forward buttons, users can only revisit the pages they have seen in that specific tab and once the tab is closed, they do not have access to those pages. Furthermore, the stack-based history used in the Back button can be misleading [16]. Similarly, visually distinguished links (although they can assist revisitation and prevent unwanted revisitation) are limited in that, first, they can sometimes interfere with the design of a website, and, second, they may only be visually distinct for a limited time, depending on the browser and the web page.

Employing spatially stable landmarks and spatial memory to assist revisitation is another body of research that falls into the category of implicit revisitation tools. Due to the influence of this field on our work, we discuss it in a separate section.

\subsubsection{Landmarks and spatial memory}

A landmark is defined by Oxford dictionary [90] as:

"An object or feature of a landscape or town that is easily seen and recognized from a distance, especially one that enables someone to establish their location."

Landmarks are stationary and prominent objects around us that serve as cues for navigation and as memory reference points. By being stationary a landmark serves as a signpost when we are navigating in its nearby environment. By being prominent, a landmark stands out and stays recognizable among other objects in its surroundings [47].

Landmarks can be part of the natural world or created by people. Mountains, lakes, trees, or any other element of a landscape that stands out from its surroundings can be used as a landmark; for example, the South Saskatchewan river in Saskatoon is a landmark that is clearly differentiable from its surroundings, and can serve as a reference for other objects around it. Human-made landmarks include prominent buildings, bridges, statues, and monuments; the Place Riel building is an example of a landmark at the University of Saskatchewan. People frequently refer to Place 
Riel as a reference point to give directions, or to remember nearby locations (e.g. Louis' pub is east of Place Riel).

The presence of landmarks also assists with the formation of spatial memory and consolidation of the location of relevant objects in memory by establishing relation to the landmark. Spatial memory is a kind of human memory that is responsible for building a cognitive map of our surrounding environment by recording locations, directions, routes, and landmarks. Without spatial memory, humans must always rely on visual search to find the location of objects [68]. Using spatial memory, rats can find food in a maze [57], and taxi drivers can find their route in London's streets [48]. Landmarks are an essential part of forming spatial memory, which help people find associations between the surrounding environment and target locations [15,69].

Researchers have looked at the use of spatially stable landmarks and spatial memory to assist revisitation. In the context of digital interfaces, researchers have shown that people use different features that are naturally available in their surroundings as landmarks to assist their spatial memory in revisitation. For example, Uddin, et al. and Schramm et al. showed that the corners of the screen or window can be a strong anchor to help people recall toolbar items $[53,73,80]$. When natural landmarks were not present, some researchers have introduced artificial landmarks to the interfaces to assist with revisitation. Artificial landmarks are visual objects that have no purpose in the system other than to provide a spatial reference. For example, Uddin et al. showed that adding a background image or set of anchor points (see Figure 2.7) can reduce task completion time and number of errors in reselecting menu items [82]. 

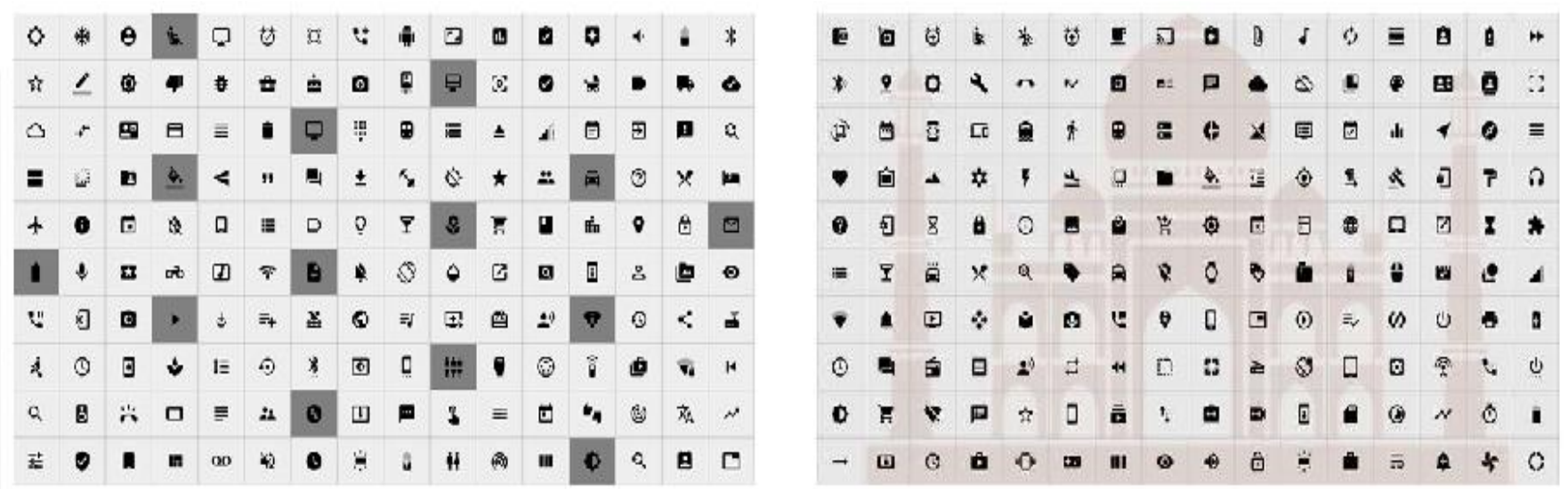

Figure 2.7. Using anchor points (grey squares in left figure) or background image (right figure) to assist formation of spatial memory

HandMark Menus (see Figure 2.8) are another example of using spatial landmarks to help with faster menu selection. This technique takes advantage of the user's knowledge of their hands and fingers, using them as artificial landmarks to locate menu commands; this is valuable in settings where natural landmarks are not present in the environment, such as large touch tables [80]. Also, Cockburn et al. introduced Space-filling thumbnails to assist with the formation of spatial memory by presenting a single page overview of the document [17]. While Space-filling thumbnails proved to be effective in short documents, their utility is questionable in long documents when a few thumbnails cannot provide a comprehensive overview of the documents. In a similar design to Space-filling thumbnails, Swifter uses a grid of thumbnails of a video during scrubbing actions to cover a higher number of frames and offer more accuracy in navigation [49]. 


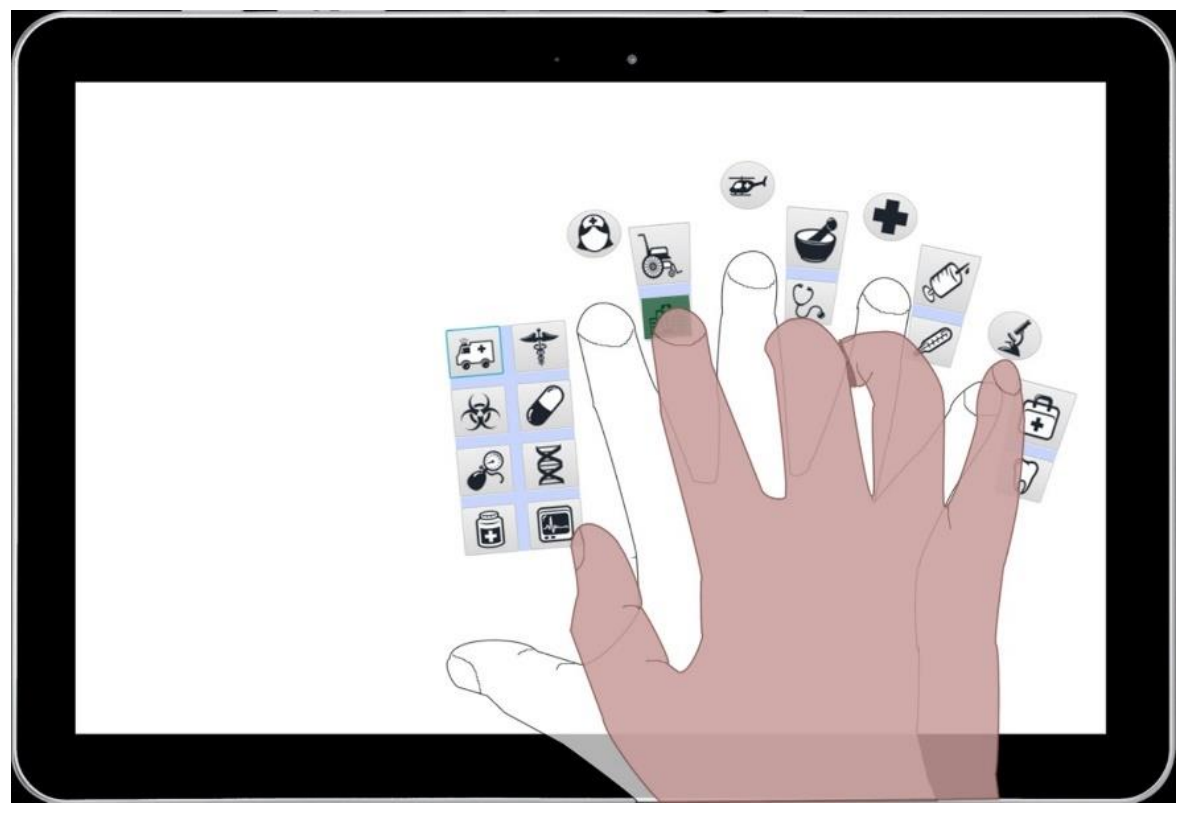

Figure 2.8. Hand as artificial landmarks: HandMark menu introduced by Uddin et al. to assist with revisitation of menu items in large tablets.

Exploring landmarks on linear navigation tools to support revisitation is another area of recent research into artificial landmarks. Uddin et al. showed that adding thumbnails (see Figure 2.9: e) and icons (see Figure 2.9: d) next to the scrollbar in a PDF document viewer and to the timeline of a custom video player (see Figure 2.9: b, c) can lead to faster revisitation compared to revisitation using a plain scrollbar or timeline [82]. Uddin reported that both icon- and thumbnailaugmented designs were faster and less prone to errors than standard unadorned scrollbars and sliders. Also, a decrease in the mean completion time and the number of errors for each interface across all blocks suggested that participants used their spatial memory instead of merely relying on visual search for revisitation. Although these designs were shown to be a promising way of helping revisitation in different types of documents, they also showed drawbacks in revisitation with long documents. For example, the 30 icons or 11 thumbnails on the scrollbar shown in Figure 10 would each cover many pages in a long document. 


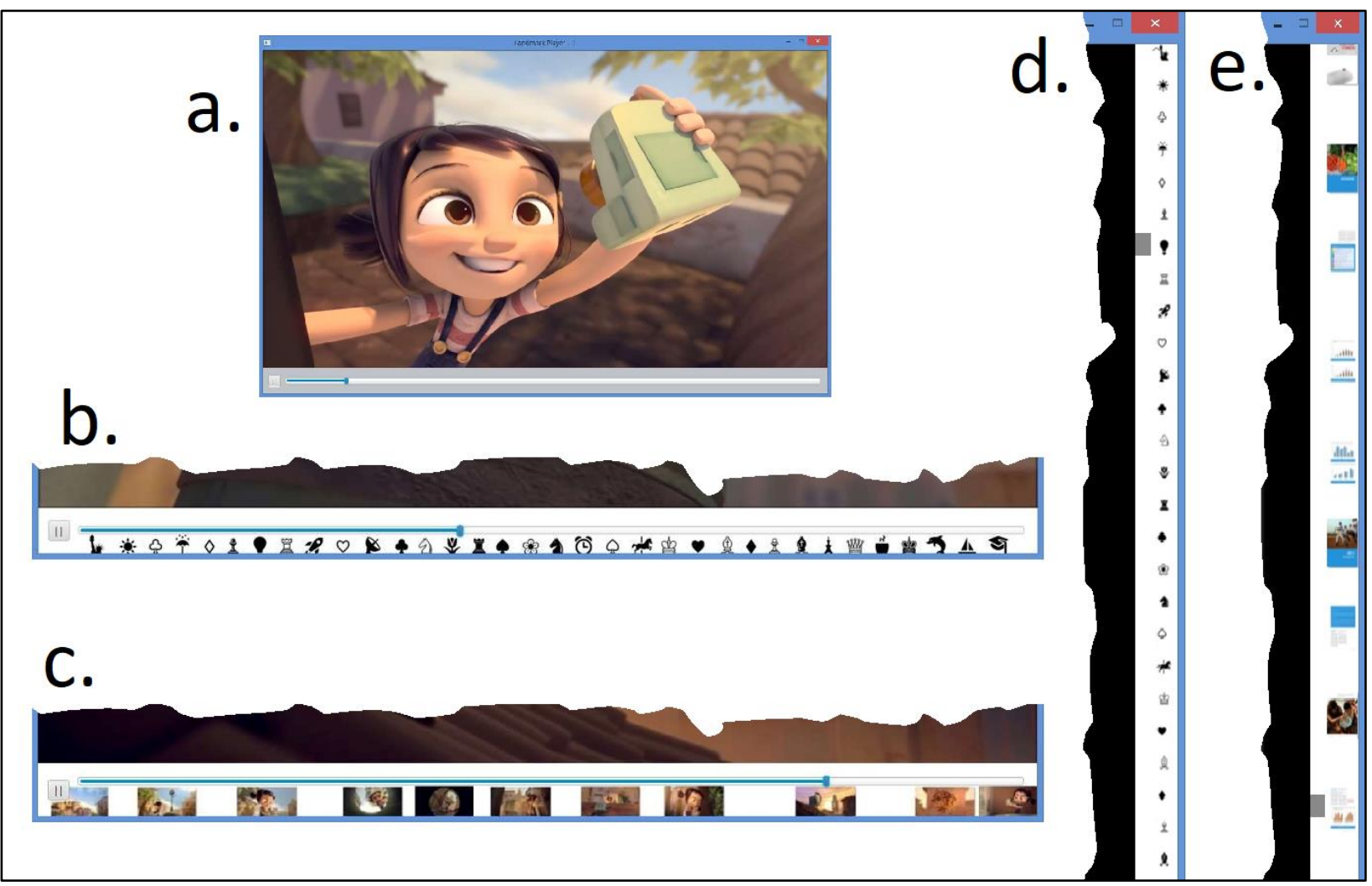

Figure 2.9. Uddin et al. scrollbar and slider augmentation. A: regular media player. B: media player with icon-augmented timeline. C: Media player with thumbnail-augmented timeline. D: Icon-augmented scrollbar. E: thumbnail-augmented scrollbar.

\subsection{SCROLLBAR AUGMENTATION}

Scrollbar and sliders are widgets introduced to help navigation with linear documents and are one of the most widely used means of digital navigation. However, since these widgets show only one portion of the content at a time, they can hamper the formation of spatial memory [82]. To tackle this problem researchers have considered using different methods of augmenting scrollbars to improve revisitation. For instance, DeLine et al. have added a miniature view of the document next to the scrollbar to help with the formation of a stronger spatial memory of the document [23]. To measure the usefulness of their Code Thumbnails, they logged the activity of five participants 
performing some tasks on computer coding and reported that all participants used their design for a faster navigation and selection in the IDE. They also indicated that Code Thumbnails comprised between $40 \%$ and $91 \%$ of all logged navigation activities of participants.

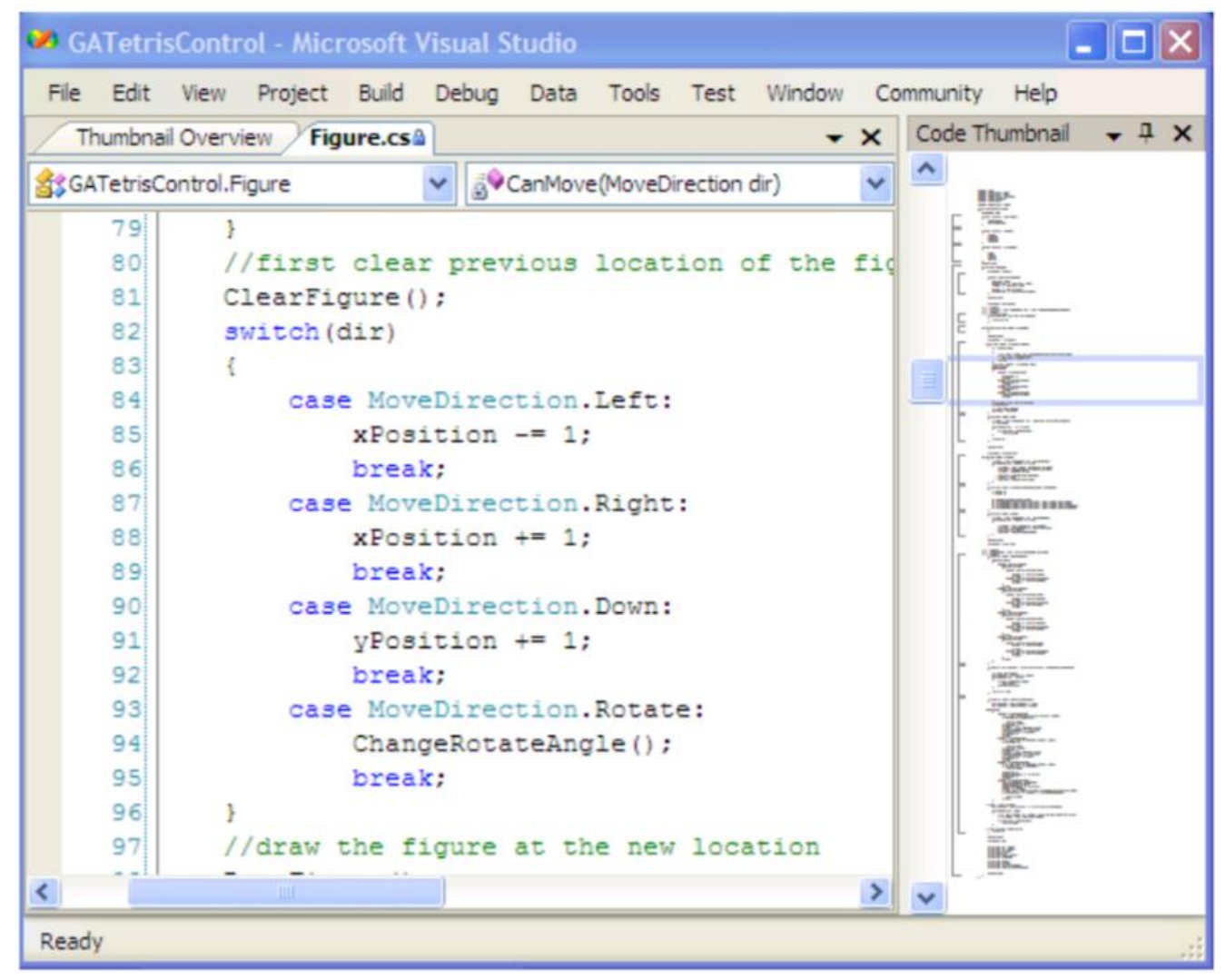

Figure 2.10. Code Thumbnail: Enhancement of revisitation by adding a miniature view of the document next to the scrollbar

In addition, many Integrated Development Environments (IDEs) for writing code are enhanced to visualize the occurrence of search terms on the scrollbar using marks of different colors (see Figure 2.11). For example, Microsoft's Visual Studio IDE uses marks on the scrollbar to highlight different kinds of errors, modification and debugging tools to make it easier for the user to navigate to these locations [91] . 

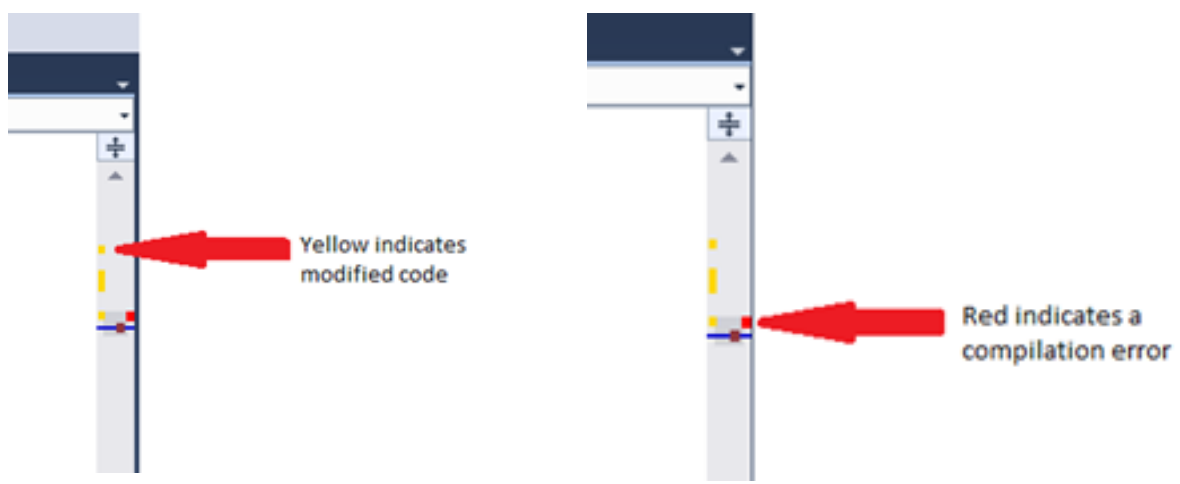

Figure 2.11. Left: yellow marks on scrollbar in Microsoft Visual Studio highlighting modified codes. Right: red marks in the same interface indicating location of complication errors.

Similarly, as described earlier, some techniques have visualized users' actions on the scrollbar. For example, Alexander et al. placed footprints of users' actions on the scrollbar [4] (see Figure 2.5: right), and Hill et al. added a histogram next to the scrollbar to show users' navigation history [4], [38] (see Figure 2.5: left).

Other researchers have also explored adding spatially stable landmarks, such as thumbnails, abstract symbols, and labels to the scrollbar to facilitate revisitation $[3,82]$. For example, Osada et al. added letters above a slider used to select a text label (positioned such that each letter indicated the start of that part of the dataset) to simplify the selection of items from a long list [63] (see Figure 2.12). More recent examples of this design can be seen in current applications such as the iPhone's contact list. 


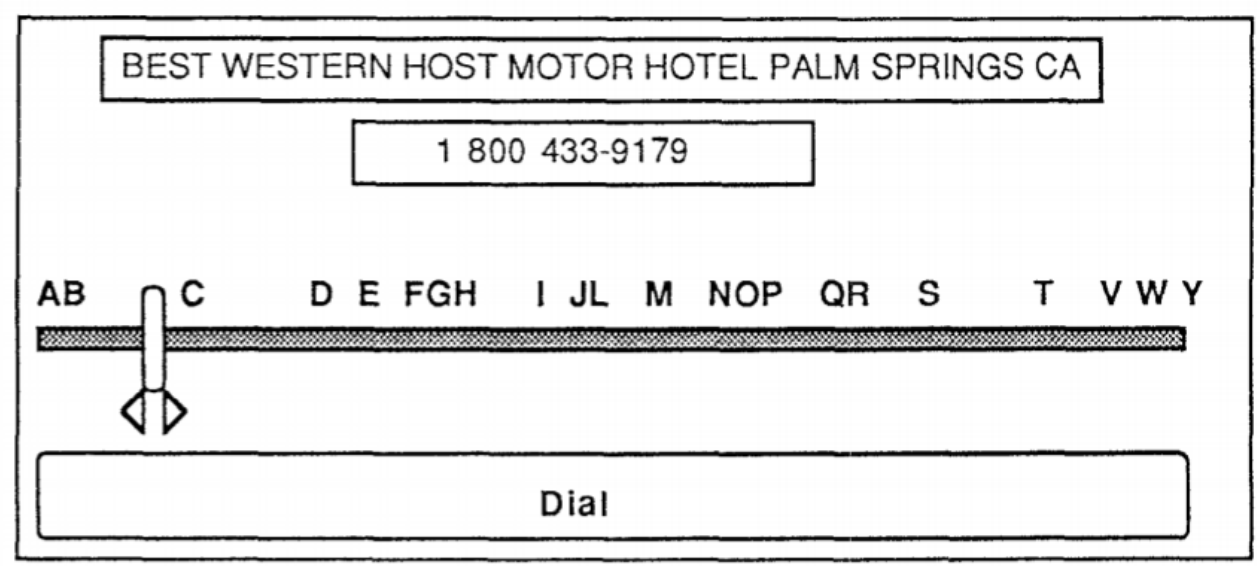

Figure 2.12. Alpha Slider: augmenting a slider with letters as landmarks

\subsection{MEMORY-BASED INTERACTIONS}

Visual search and memory-based interactions are two of the most important interaction methods in digital interfaces. When selecting an item from a list or navigating to part of an interface, people usually rely on visual search, their memory, or a combination of both. Visual search involves looking for a particular target among other distractors. Examples of visual search can be found in many aspects of our daily life. When scanning different isles of a supermarket for a specific item for the first time, we use visual search to find the item of interest in a shelf. Selecting the same item from the same shelf in future, however, will not be purely a visual search. By visiting a place or interacting with objects in our surroundings, we create a mental map of that environment; this mental map is a kind of spatial memory and thus enables a memory-based selection.

Memory-based selection, as its name suggests, relies on the power of human memory to find a target or limit the search area. In the supermarket example, if we recall that the desired item was on the third shelf of the eighth aisle, we can then limit our visual search to that specific location rather than the whole supermarket. Using the power of memory-based selection has been heavily investigated $[9,33,34,44,70,72]$, and the outcome of this research shows the performance 
advantages of memory-based selection over visual search. Even though there are different types of memory that can help with memory-based selection, in this thesis we focus on spatial memory.

Spatial memory, as introduced in section 2.3.3, is knowledge about the location of objects in a surrounding environment or an interface. Experience that is gained by interacting with visually stable landmarks helps people retrieve the location of elements on screen after practice. Researchers have also investigated memory of spatial gestures in marking menu [44] and flower menus [9] , and showed that the selection time using these interaction methods decreased with practice. This decrease in selection time with practice, also known as "novice to expert" transformation, indicates that as participants become more familiar with the items, they tend to rely on their memory to select an item instead of doing a time-consuming visual search.

The Data Mountain visualization [70] introduced by Robertson et al. provides a 3D environment for users to place a thumbnail of a web page on a virtual slope. The researchers carried out a study involving several blocks of find-and-select tasks and reported a decrease in mean completion time of each revisitation, indicating the formation of spatial memory. Robertson's design helped users take advantage of spatial memory and landmarks available in the virtual environment to find desired document faster compared to a pure visual search.

Arranging menu items in a spatially stable setting to assist with formation of spatial memory is another well-studied area. ListMap [33](see Figure 2.13:middle), CommandMap [72] (see Figure 2.13:left), and FastTap [34] (see Figure 2.13:right) are all interfaces that present a large set of commands or items in a spatially stable interface to ease formation of spatial memory. Studies conducted on all of these interaction methods show a significant advantage in performance compared to regular lists and hierarchical menus when revisiting items. 

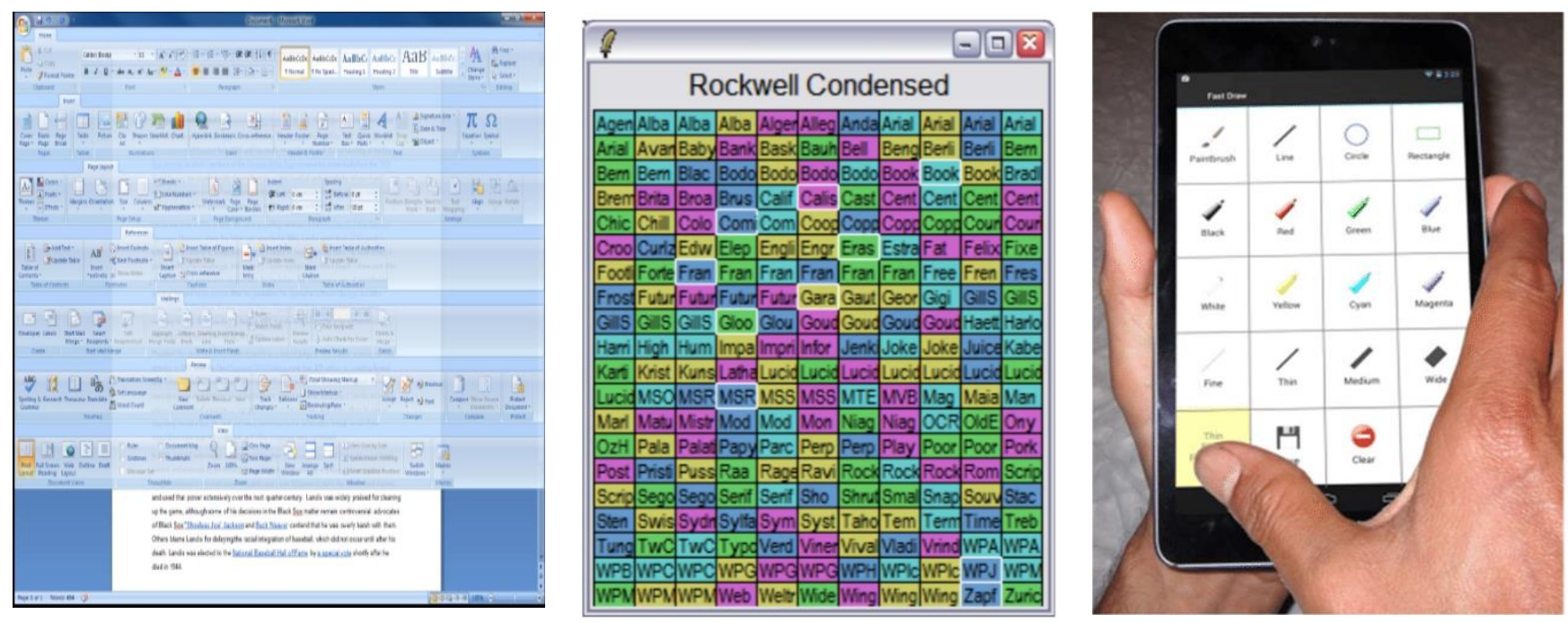

Figure 2.13. Left: CommandMap, Middle: ListMap, Right: FastTap. Utilizing spatially stable menu icons to assist formation of spatial memory

\subsection{MEMORY DEVICES IN DIGITAL INTERFACES}

Mnemonic devices are techniques invented to help people learn and recall information by associating them with meaningful objects. Some popular mnemonic devices are as follows:

\subsubsection{Imagery mnemonics}

Imagery mnemonics ease the process of memorizing and recalling a list of items by creating an association between an image and the items that need to be memorized. The Method of Loci, invented by the ancient Greeks, is one of the oldest methods that uses images and spatial learning to make information easy to memorize [66]. In this method, a person imagines a familiar place, such as his or her home, and then relates items or places in the home with the objects to be remembered. Figure 2.14 gives another example of a representative mnemonic that helps people remember how many days are in each month of the year - the knuckles of a person's two hands can represent the longer and shorter months. 


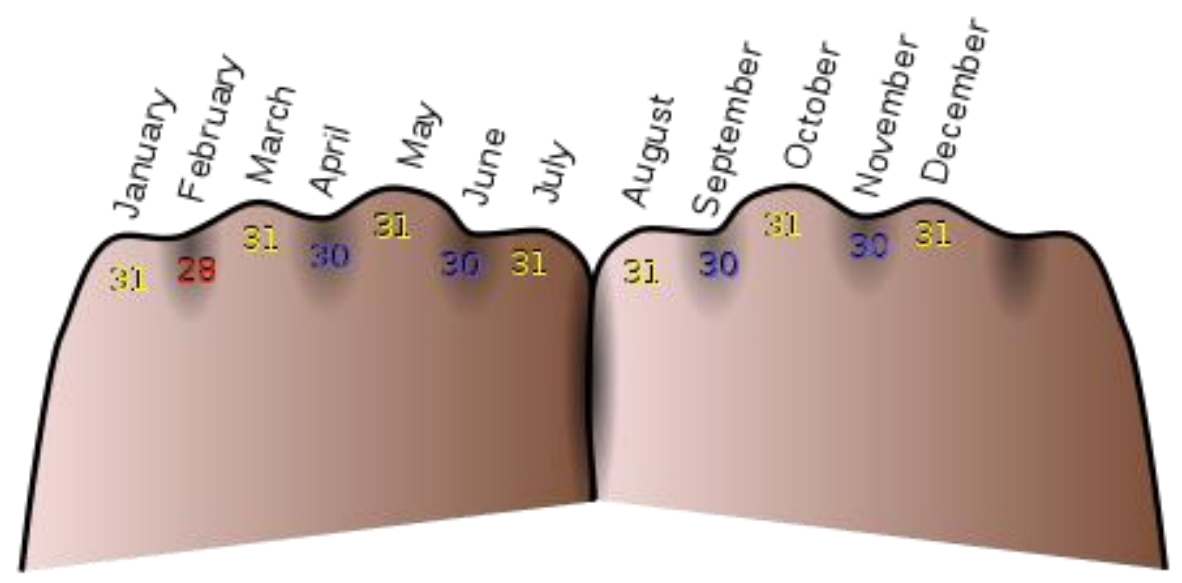

Figure 2.14. Use of Mnemonic devices to assist memorization

Mnemonics have been shown to be highly effective in learning at colleges and universities [51], and in learning new languages $[5,6,24,43]$, and are also widely used in computer interfaces. Icons are the most popular mnemonics used in digital interfaces and are a representation of data, functions, and applications.

Icons' potential to serve as metaphors and their universal comprehensibility play a crucial role in their widespread applications in digital interfaces. Icons as metaphors enable ease of understanding by creating associations between them and real-world objects and concepts [31]. For instance, the metaphor of the Desktop was introduced by the invention of GUI to help people understand concepts such as files, folders, recycle bin, and inbox, by making an association between the objects in a physical office and different functionalities that a computer serves.

Moreover, universal clarity of icons on many occasions make them more recognizable and less prone to open interpretation $[29,65,74]$. For instance, in most GUIs a button with an ' $\mathrm{X}$ ' symbol is presented as exit button. Icons can thus be a common visual language to tackle the problem of comprehensibility of ideas. Furthermore, icons' potential to convey information concisely, compared to other more descriptive media, makes them a desirable solution when screen space is constrained. Previous studies on icons as imagery mnemonics proved their usefulness as a 
replacement for labels in digital interfaces when space is a concern. Studies showed that icons can reduce the number of errors $[31,58]$ without sacrificing task completion time $[58,86]$.

Finally, the "picture superiority effect" is an idea suggesting that imagery mnemonics can be highly effective in assisting memory. The picture superiority effect refers to humans' ability to recognize, memorize, and recall images faster than words. There are numerous studies done to investigate which stays longer in memory, an image or a word [22,40,75,85]. Shepard showed that when recognizing 600 items from a list of words, sentences, and pictures, participants had $98 \%$ chance to recognize old stimuli when it was a picture and 90\% when it was a word [75]. In a similar study, Curran et al. asked participants to recognize some repeated words from a list of 20 photos and 20 words which were previously seen by participants. They saw that participants had a higher accuracy detecting repeated images compared to repeated words [22].

However, using mnemonics in GUIs is not limited to icons. For instance, Uddin et al. used a background image to improve revisitation time for item selection [82] (see Figure 2.15). In their designs, Uddin et al. showed that imagery mnemonics can help with revisitation by facilitating the formation of spatial memory. Also, Giannisakis et al. improved shortcut accessibility by forming connections between toolbar button icons and their corresponding shortcut [30].

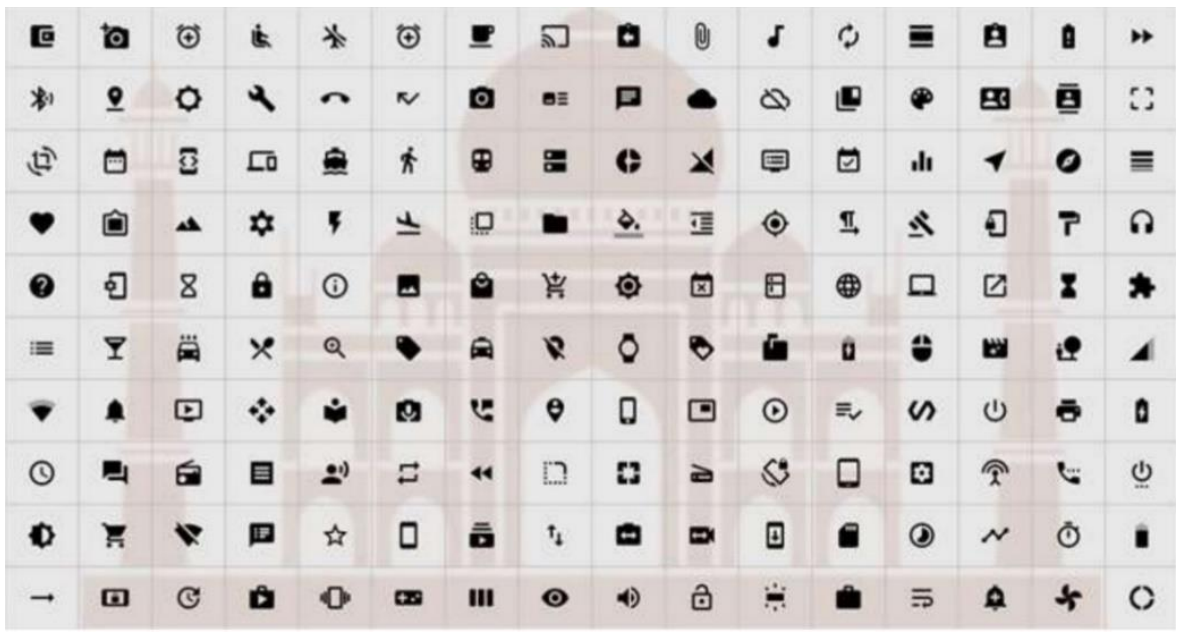

Figure 2.15. Use of an image as a mnemonic device to help with item selection from a menu. 
It is important to note that artificial landmarks often use images that are unrelated to the content of the document - in these situations, the landmarks are not suggestive (as with icons in the desktop metaphor) but can still be used to form an abstract mapping (as with the Method of Loci).

\subsubsection{Letters as mnemonic devices}

Acronyms and Order mnemonics are the two most popular mnemonic devices that are mostly used with letters. These devices, similar to Imagery devices, aid memorization of ideas and concepts by making a connection between familiar pieces of knowledge and new things that need to be learned. For example, we use Acronyms which are words that are formed by combing the first letter of each word or phrase that need to be remembered and recalled. Roy G. Biv is a name used to make the colors of the rainbow easier to memorize: Red, Orange, Yellow, Green, Blue, Indigo, and Violet; Or, FANBOYS is used to teach English language learners the seven coordinating conjunctions (For, And, Nor, But, Or, Yet, So).

Order mnemonic devices help memorization of a list of elements when the order in the list does matter. In this technique, people form a sentence using the first letter of each name in the array. For instance, The seven levels of the United States Constitution and their order of hierarchy (Legislative, Executive, Judicial, Supremacy, Amendment, Statehood, Ratification) can be memorized using this sentence "Large Elephants Jump Slowly And Sink Rapidly". Another

illustration of this method is mentioned in Figure 2.16, where we used the sentence "My Very Easy Method Just Speeds Up Naming Planets" to memorize a list of planets in their respective order. 


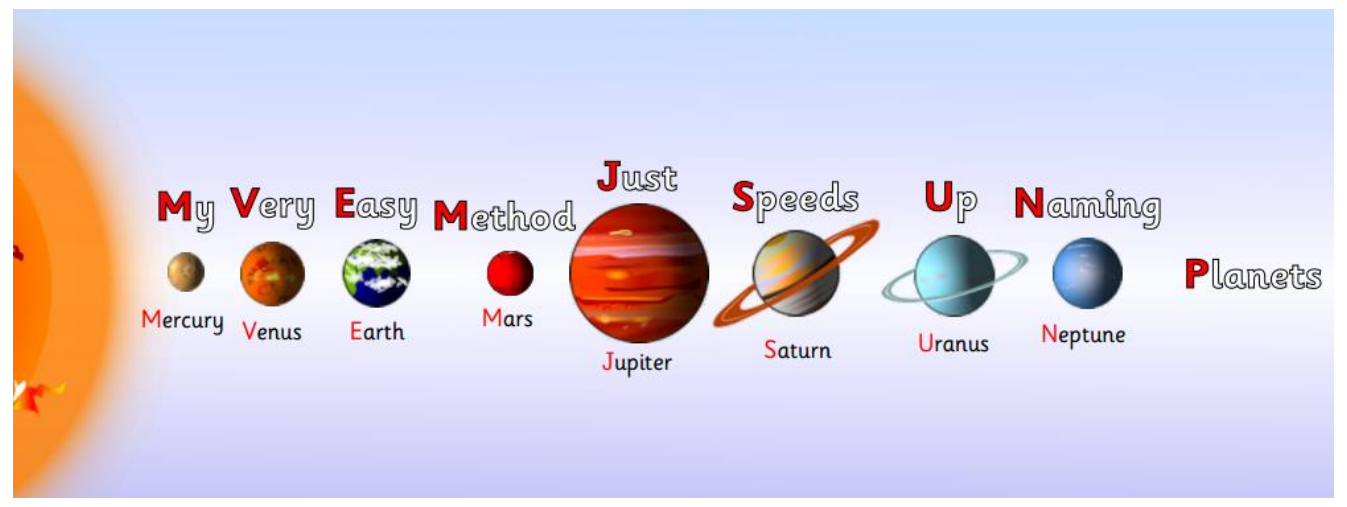

Figure 2.16. Using Order Mnemonics to remember the order of the Solar System planets

\subsubsection{Chunking as a memory device}

Chunking is popular a way of organizing larger pieces of information into a smaller number of items, to facilitate retention in memory. In this method, we take advantage of our natural tendency to see patterns and make connections between them [11]. Research has shown the usefulness of chunking - for example, in making series of numbers easier to retain and recall $[12,20,21,27,28,35,39,60,61,64,71]$. Memorizing phone numbers and pin codes are perhaps the two most common uses of chunking in daily life. In this method, instead of memorizing an 11digit number as the eleven digits 3069664886, people break it down to three chunks: 306-9664886. 


\section{CHAPTER 3}

\section{THE DESIGN OF A TWO-LEVEL ARTIFICIAL- LANDMARK SCROLLBAR}

In this chapter we introduce the Double-Scrollbar interaction method, inspired by Uddin's et al. augmented scrollbar [82]. Then, we explain the implementation and provide design details of three different versions of the Double-Scrollbar. This chapter is intended to make readers familiar with the interface and lay a foundation for the evaluation process in the next chapter.

\subsection{LANDMARKS FOR LINEAR DOCUMENTS}

In Chapter 2, we discussed the importance of addressing revisitation in digital contexts, and provided examples of tools that have been developed to facilitate revisitation. While each of these tools are useful in particular cases, all of them show drawbacks for revisitation in long documents: search bars need the user to recall exact queries; bookmarking interrupts people's train of thought and requires several steps; history lists become long and cluttered and ask for a time-consuming visual search each time user is looking for an item; recency lists are limited in that they can hold only a limited number of items and if the item is not in the list the cost of a visual search will be added to revisitation process; Read Wear, which marks locations based on user behavior, can capture false points of interest.

Our goal is to use the power of landmarks and spatial memory to address problems of revisitation in digital contexts. Landmarks are prominent objects in an environment or an interface that can serve as signposts and reference points to find other objects. Landmarks aid formation of spatial memory by consolidating a mental map of environment in a person's mind. Formation of spatial memory facilitates memory-based interactions which are reported to be faster and requires less cognitive load compared to a pure visual search $[9,33,34,44,70,72]$. Being faster and posing less 
cognitive burden encouraged many researchers to use the power of this type of interaction in user interfaces to address problems involved with revisitation.

The starting point for our designs is the work of Uddin et al. who explored the possibility of adding landmarks next to linear controllers to facilitate the formation of spatial memory and provide a faster revisitation experience [82] (see Figure 3.1). Uddin et al. in his design showed that the adjacency of scrollbar handle or slider handle to an icon or thumbnail can assist recalling a location and navigating to a specific part of a linear document. In a standard scrollbar, when users try to memorize their current position in the document using scrollbar, they estimate the distance of the scrollbar handle to the nearest natural landmark (e.g., the top or bottom of the scrollbar). When the document is long and requires a pixel-precise selection, this inaccurate estimation leads to many scrollbar interactions. Uddin's et al. artificial landmarks provide more opportunity to form an association between icons and the document content and improve support for revisitation by forming stronger spatial memory.

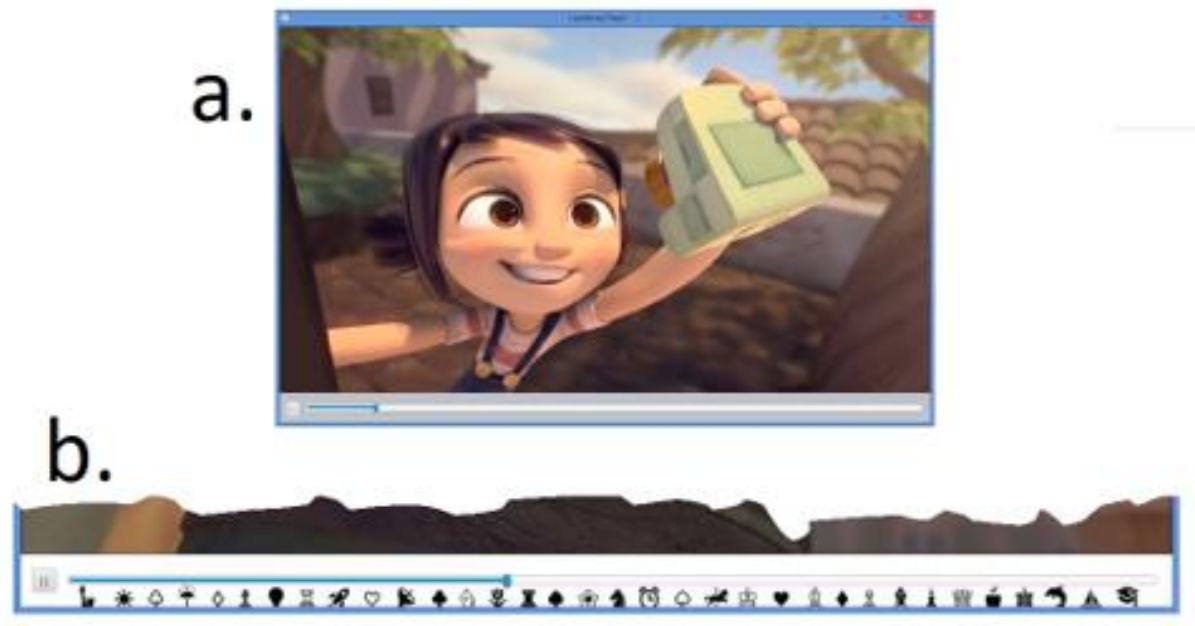

Figure 3.1. Uddin et al.'s artificial-landmark augmented movie slider 


\subsection{PROBLEM: LANDMARK DENSITY IN LONG DOCUMENTS}

Even though Uddin's design was shown to be effective in short documents and videos, its usability for long documents is questionable. One reason we believe this design is not effective in long documents is the limitation in the number of landmarks we can add to the scrollbar. Since the size of scrollbars and sliders are fixed by the window or display size, the number of icons that can be placed alongside a scrollbar or slider is constrained. Therefore, one icon has to represent many pages or much of a video which makes the association between icons and content harder and development of precise spatial memory more difficult.

The other possible reason for the inefficiency of the one-level augmented scrollbar is the lack of necessary pixel precision in the moving of the scrollbar handle to get to the desired location in a document. In long documents, a slight movement of scrollbar handle leads to a several-pages navigation in the document, requiring users to make several adjustments to get to the desired location in the document. These adjustments make navigation difficult and time-consuming and lead to a user frustration.

\subsection{USING A DOUBLE SCROLLBAR}

The two-level landmark-augmented scrollbar aims to tackle two problems with the single-level design. As mentioned in section 3.2, difficulty of navigation is one of the major setbacks of a single scrollbar. When the document is long, dragging the scrollbar handle cannot be an optimal strategy to move to an area of interest, as each slight movement of scrollbar handle would move the document many pages up or down. Therefore, we examined the idea of multiple-granularity selection - an idea that already exists in some GUIs such as a date picker where users first pick a year (coarse selection) then a month, and finally a day (finer granularity). In Double-Scrollbar method, we partitioned the coarse-grained scrollbar and assigned 1/30 of the content to each partition, then we provided fine-grained navigation within each partition using a fine-grained scrollbar. 
We augmented both scrollbars with landmarks to enable precise spatial memory. In Uddin's design, when the document is long, each icon or thumbnail on the scrollbar presents many pages of the document which hampers the association between landmarks and the content. This association is crucial in forming spatial memory and memory-based interactions. The introduction of two sets of landmarks to the scrollbars enables an association between the content and a combination of two landmarks and increases the number of possibilities to $n^{2}$ when $n$ is number of icons on one of the scrollbars (e.g. we added 30 icons to each scrollbar for our experiment which produced 900 combination of icons on the scrollbar).

\subsubsection{How does the Double-Scrollbar work?}

We divided our 900-page contents into 30 blocks of data with equal numbers of pages (30 pages in each block). A block in this thesis refers to a fixed number of pages that are clustered together (e.g., represent a chapter of a book). After clustering our data into 30 blocks, we added 30 icons of equal size to the coarse-grained scrollbar. Icons were vertically distributed and evenly spaced over the coarse-grained scrollbar and were mapped to one block of content in the document (see Figure 3.2).

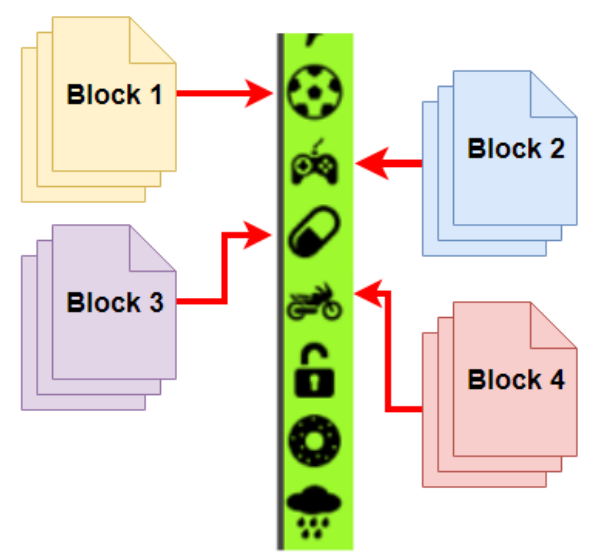

Figure 3.2. Assigning one block of content to each icon on the coarse-grained scrollbar

Clicking on an icon in the coarse-grained scrollbar will take users to the beginning of the corresponding block in the document. For instance, the first icon was mapped to page 1, the second 
icon to page 31, the third icon to page 61, all the way down to the beginning of the last block (see Figure 3.3). Then, to allow fine-grained control within each block, we added another scrollbar to the right of the coarse-grained scrollbar. The fine-grained scrollbar also has 30 landmarks, each of which is mapped to one subset of that block. Therefore, in our 900-page document if users want to navigate to page 68 , they have to first click the third landmark on the coarse-grained scrollbar and then the eighth landmark in the fine-grained scrollbar.

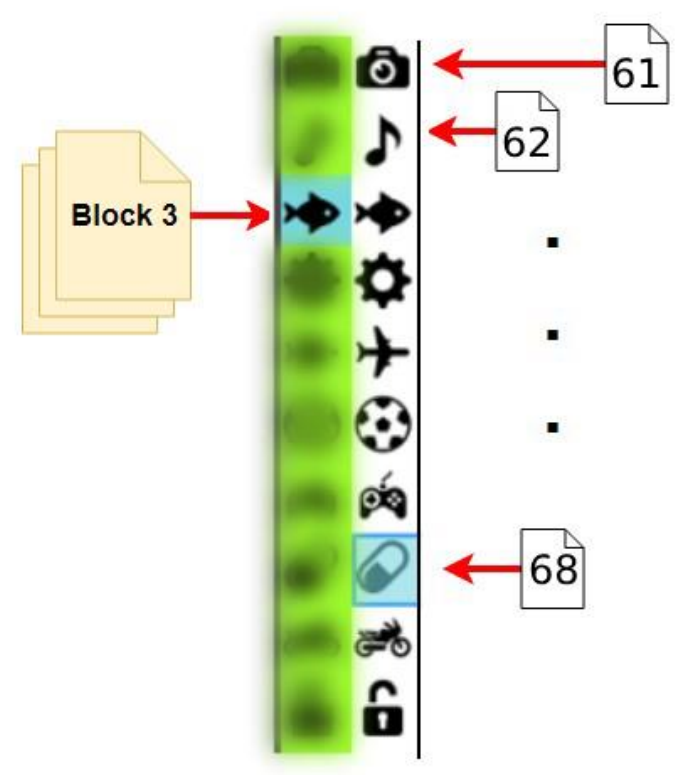

Figure 3.3. The arrangement of pages in each block

Selected landmarks on both scrollbars are denoted by a blue background color that distinguishes them from the rest of the landmarks. For the fine-grained scrollbar, there is a handle that shows users' position in that block similar to scrollbar handle in a regular scrollbars.

For the purpose of the experiments described in later chapters, five interfaces were developed using web technologies (HTML and JavaScript), using the Chrome browser. Also, to avoid loading the 900-page document at once, we used asynchronous JavaScript requests (Ajax) to load data for each corresponding block on demand. Loading each block of content on demand ensured that the 
application did not overburden to the processor and avoided invalid completion time values because of lag in the browser during navigation.

To customize the design of the scrollbars for each interface, and also to enhance it with logging functionality, we hid the browser's real scrollbars and designed a new interface widget that could be added to web applications. Interaction with the widget was different for the coarse and fine scrollbars: on the coarse-grained scrollbar, users can only click on each landmark to go to a certain block; for the fine-grained scrollbar, however, clicking and dragging are added. In addition, using the mouse scroll wheel will take users up and down on the fine-grained scrollbar. If they are at the beginning or the end of the block, scrolling takes them to the next block.

\subsubsection{Questions to be answered about the feasibility of Double-Scrollbar}

To test the practicality of the Double-Scrollbar, we had to answer some questions regarding its usability. First, in Uddin's et al. design, users had to memorize one icon for each target, while in the Double-Scrollbar, a combination of two landmarks must be memorized. Memorizing a combination of two landmarks introduces a tradeoff for providing ease of navigation in return for more cognitive load.

Second, among many different types of landmarks, which are best in facilitating the formation of spatial memory? In the following section we propose using icons, letters, and digits as three common landmarks in current interfaces and then, in Chapter 4, we compare them to find the answer to the second question.

Third, is our design faster and more accurate than the current unadorned scrollbar and Uddin et al.'s single-level design? To answer this question, we conducted two studies to compare these designs; results of these studies are laid out in Chapter 4.

Finally, is the Double-Scrollbar easy to work with and favored by users? We answered this question by collecting data from different surveys, measuring user's personal preferences, and perceived workload; these results are also part of the studies reported in Chapter 4. 


\subsection{DESIGN ISSUES}

\subsubsection{Number and density of landmarks}

In our design, we augmented each scrollbar with 30 icons. Taking our screen size and pixel density into account, we examined several icon densities and decided that 30 icons is the maximum to fit in the screen and keep icons easily recognizable.

\subsubsection{Interaction with the two-level Scrollbar}

As introduced above, the interaction methods for the two scrollbars were different. For the coarsegrained scrollbar, users could only select a landmark by clicking on it. Clicking a landmark loads a block of content (resembling a chapter of a book) that can be explored more with the fine-grained scrollbar. The fine-grained scrollbar had all the functionalities of a regular scrollbar, except that it was augmented with landmarks. Interaction methods on the fine-grained scrollbar included clicking on the scrollbar, dragging the scrollbar handle, and using the mouse wheel to navigate in the loaded block. Note that scroll buttons at the top and bottom of the scrollbar were not included in the design.

In our design, we did not assign each page an icon combination. Instead, we divided the document into 900 chunks and assigned a combination of icons to each chunk. This means that a chunk can be shorter or longer than a page. For example, when the document is 300 pages, each pair of icons will be mapped to $1 / 3$ of a page which adds more precision to the selection.

As for documents longer than 900 pages (or long series of information such as genome sequences as discussed in section 5.3.1), we could add another level of landmarks. Using a third level of landmarks will increase the number of possible combinations up to 27000. However, adding another level of landmarks could be unfavourable since it increases users' cognitive load, and requires more research on usability. This design was not tested in our studies. 


\subsection{FIVE SPECIFIC SCROLLBAR DESIGNS}

We developed and tested five document viewer interfaces: four of them used landmarks, and one replicated a standard scrollbar design.

\subsubsection{The standard scrollbar: Single-Scrollbar with no landmarks}

The Standard design represents a standard interface with a single unadorned scrollbar at the right side of the screen (see Figure 3.4). This scrollbar has most of the features that a typical scrollbar offers, including: scrolling with the mouse wheel, dragging the scrollbar handle, and clicking on the scrollbar for navigation.
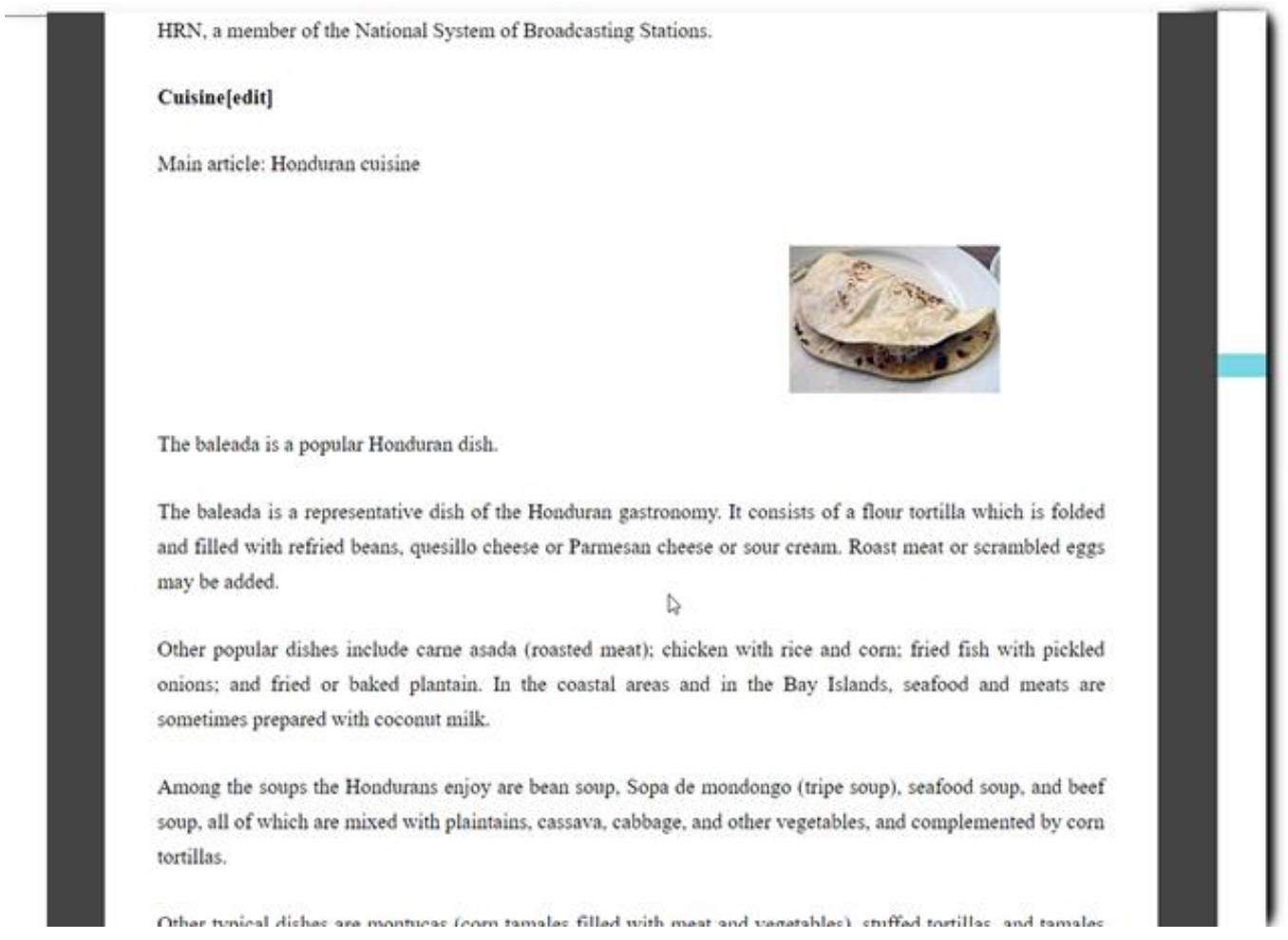

Figure 3.4. Study interface: Standard unadorned scrollbar 


\subsubsection{The Single-Icons design: Single-Scrollbar with icon landmarks}

Single-Icons is an artificial-landmark augmented interface with a single scrollbar (width 31px, height 954px). Thirty icons (27x27px; monochrome) are added vertically (see Figure 3.5) next to the scrollbar [82]. The icons are abstract and not related to the content of the document (icons remain the same for all documents). Figure 3.6 a shows a list of icons used for this interface.
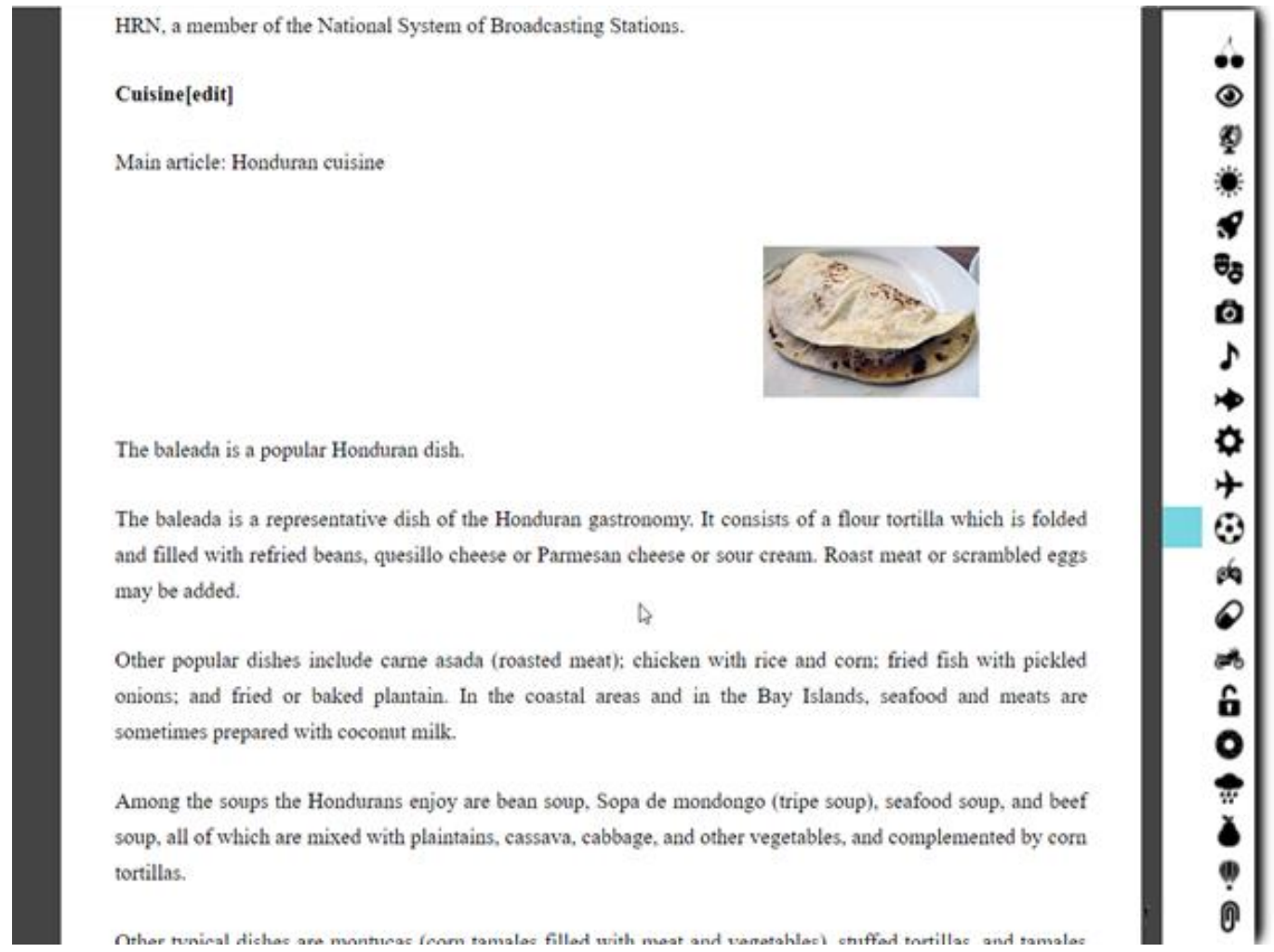

Figure 3.5. Study interface: Single-Scrollbar augmented with icons as landmark

In a standard scrollbar, when users try to memorize their current position in a document, they try to estimate the distance of scrollbar handle to the nearest landmark (e.g., the top or bottom of the scrollbar), which is not precise most of the time and leads to many scrollbar interactions to revisit the right page. As suggested by previous research $[5-7,24,43]$ the presence of imagery on the scrollbar could provide landmarks to assist the formation of spatial memory, allowing users to form an association between icons and content. Also, landmark discretizes the continuous scrollbar and provide reference points for memorization. 


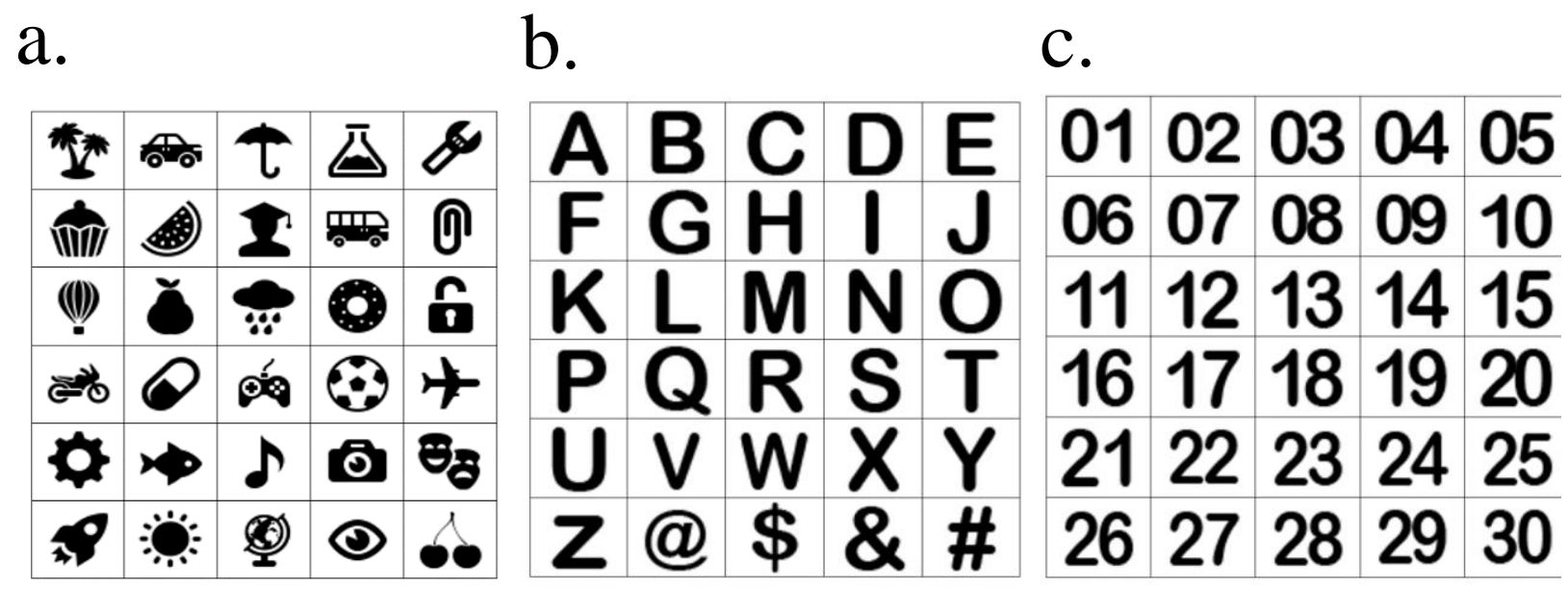

Figure 3.6. Three type of landmarks placed on scrollbar(s). a: List of icons used for SingleScrollbar and Double-Icons interface. b: Letters used for Double-Digits interface. c: List of numbers used for Double-Digits

\subsubsection{The Double-Icons design: two scrollbars with icon landmarks}

The Double-Icons design introduces a fine-grained scrollbar (31px wide and 954px tall in a 1080p monitor, highlighted in green) to provide more specificity. Each of the two scrollbars is augmented with 30 icons (see Figure 3.7). The combination of two icons requires more work from the user to form the association, but provides finer-grained access to the document (see Figure 3.6 a for the list of icons used in this interface). 


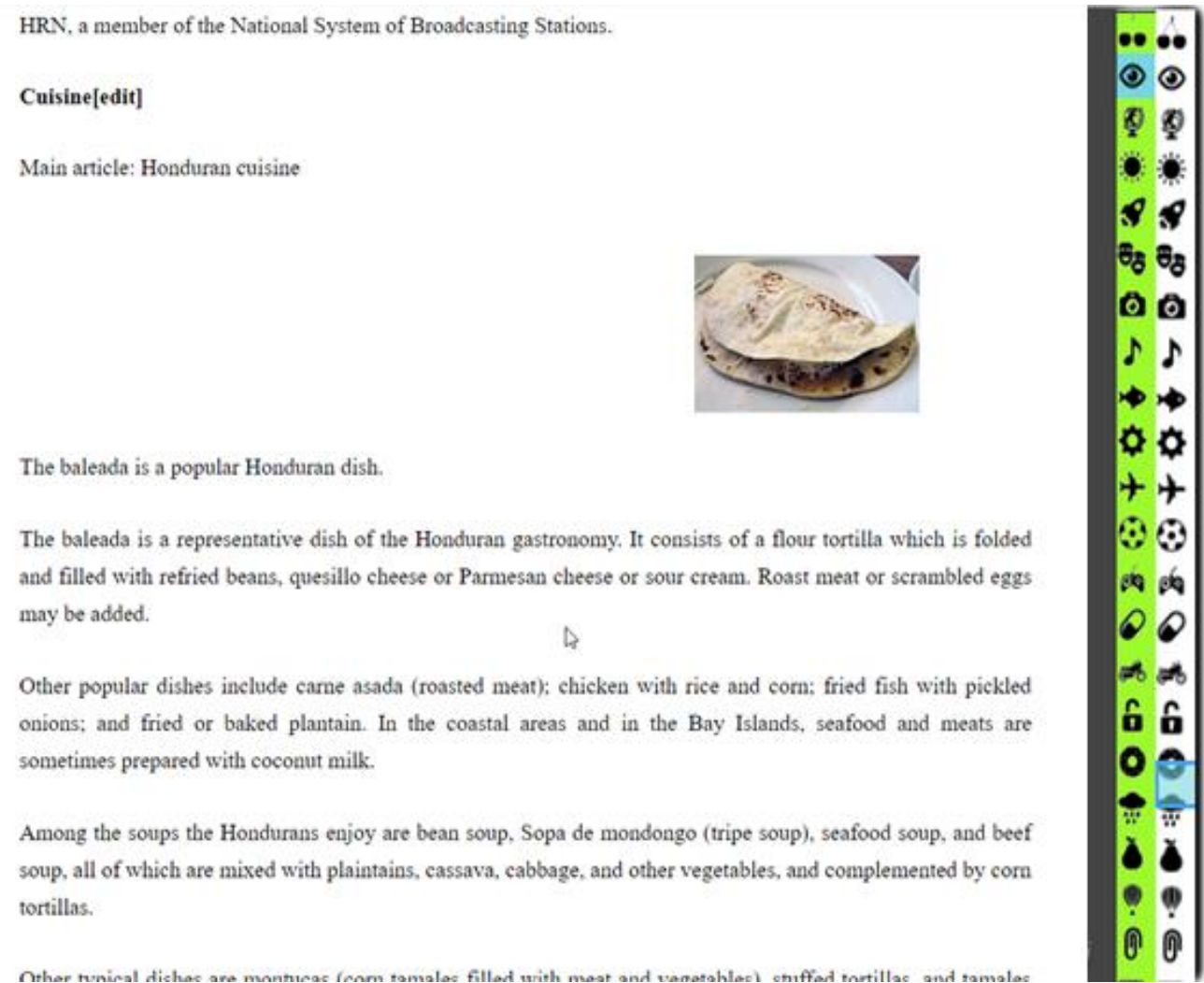

Figure 3.7. Study interface: Double-Scrollbar with icons as landmark

\subsubsection{The Double-Letters design: two scrollbars with letter landmarks}

The Double-Letters design has a similar interface to the Double-Icons design, but with letters as landmarks (see Figure 3.8). Previous work shows that letters can provide strong navigational support [3,87], and are often used in alphabetically-organized documents (see Figure 2.2: left). This design uses 26 English letters (sorted in ascending order) and four symbols: @, \$, \&, and \# (all Arial Rounded font; 27x27px). Other features are similar to the Double-Icons design. See Figure 3.6 $\mathrm{b}$ for the list of letters used for this interface. 

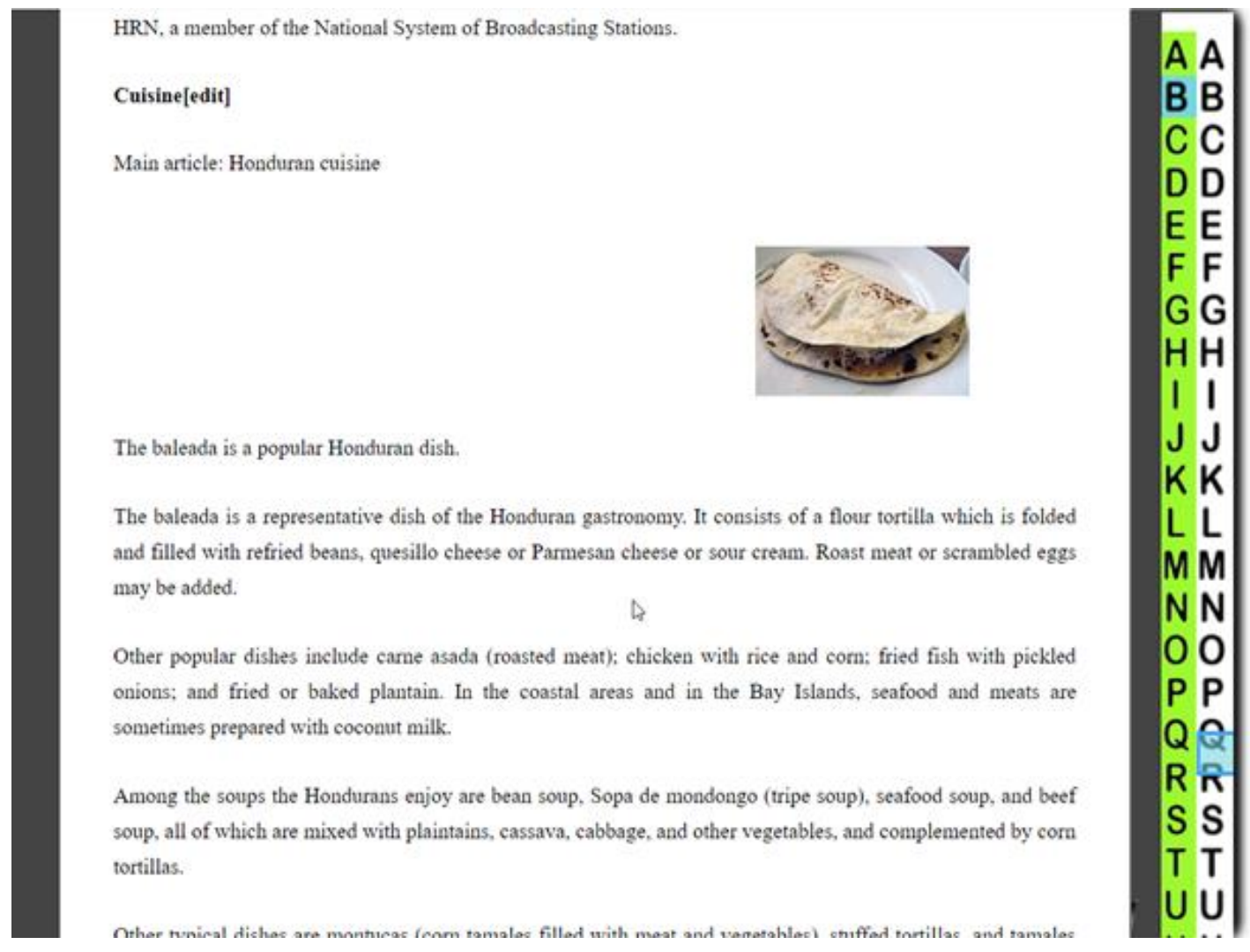

Figure 3.8. Study interface: Double-Scrollbar with letters as landmark

In this design, users form associations between document content and the two arbitrary letters corresponding to the coarse and fine scrollbar locations. Although the letters are arbitrary, however, users can often create a meaningful association by choosing elements of the content that match the letters in the scrollbars.

\subsubsection{The Double-Digits design: two scrollbars with digit landmarks}

Numbers can also be used as landmarks and are obviously used as page numbers in most documents; people also commonly remember number combinations such as passcodes or PINs. The Double-Digits design has 30 digits as landmarks from 01 at the top to 30 at the bottom (see Figure 3.9). The Arial Rounded font is used for digits (Figure 3.6 c) and other features remain unchanged. 
HRN, a member of the National System of Broadcasting Stations.

Cuisine[edit]

Main article: Honduran cuisine

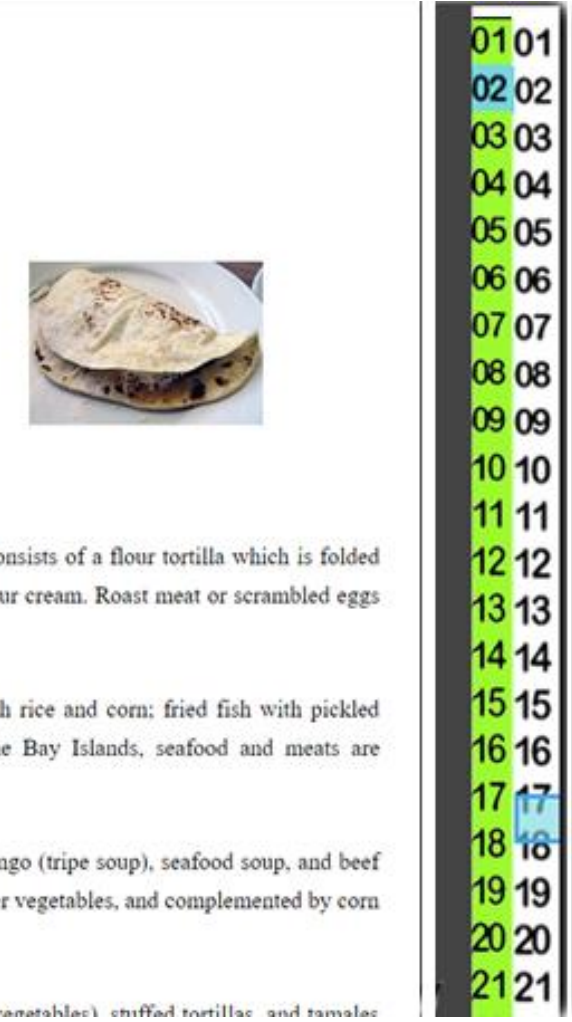

The baleada is a popular Honduran dish.

The baleada is a representative dish of the Honduran gastronomy. It consists of a flour tortilla which is folded and filled with refried beans, quesillo cheese or Parmesan cheese or sour cream. Roast meat or scrambled eggs may be added.

\section{A}

Other popular dishes include came asada (roasted meat); chicken with rice and corn: fried fish with pickled onions; and fried or baked plantain. In the coastal areas and in the Bay Islands, seafood and meats are sometimes prepared with coconut milk.

Among the soups the Hondurans enjoy are bean soup, Sopa de mondongo (tripe soup), seafood soup, and beef soup, all of which are mixed with plaintains, cassava, cabbage, and other vegetables, and complemented by corn tortillas.

Figure 3.9. Study interface: Double-Scrollbar with digits as landmark

In all of the landmark-augmented interfaces, the goal is to enable users to build an association between the page of interest and the landmarks in the scroll bar(s). Previous work has shown that strategies such as the 'Method of Loci' are an effective way to memorize even abstract information $[67,87,92]$.

To evaluate these scrollbar designs, we designed two studies described in the next chapter. The first study compared the augmented designs to a standard scrollbar, and the second study focuses on the new designs, asking whether two columns of artificial landmarks result in better revisitation performance, and which type of landmark is best. 


\section{CHAPTER 4}

\section{EVALUATION OF TWO-LEVEL LANDMARKED SCROLLBARS}

In CHAPTER 3chapter 3, we introduced the two-level scrollbar augmented with artificial landmarks as a solution to problems with revisitation in long documents. In this chapter we provide detailed performance information obtained from two separate studies that compared our designs, Uddin et al.'s one-level augmented scrollbar, and a standard unadorned scrollbar.

First, in a preliminary study we evaluated the three Double-Scrollbar designs, the Single-Scrollbar with icons as landmark, and the Standard scrollbar, in terms of task completion time and number of scrollbar interactions. The results of this study showed clear advantages for the landmark-based scrollbars, and convinced us to exclude the Standard scrollbar from the main study.

Second, in the main study we evaluated the effectiveness of different landmarked scrollbars in terms of task completion time, scrollbar interactions, and the number of times the user was unable to find the page. We also analyzed these measures in terms of distances to the target, the type of page content, and landmark proximity to scrollbar handle. The questions that we try to answer by the end of this chapter are these: Is the Double-Scrollbar better than the Single-Scrollbar? What type of landmark best supports the formation of spatial memory?

\subsection{STUDY 1: COMPARISON OF STANDARD SCROLLBAR TO LANDMARK AUGMENTED SCROLLBARS}

To measure revisitation performance of different versions of the Double-Scrollbar against Uddin's single-level scrollbar and the Standard scrollbar, we ran a small preliminary study. The goal of the study was to find out whether there are major differences between landmarked and nonlandmarked scrollbars, and therefore to determine whether the standard scrollbar should be 
included in the main study. In our preliminary study we measured revisitation performance by recording users' revisitation time and the number of interactions they made for each successful or failed revisitation.

\subsubsection{Method}

\subsubsection{Experimental conditions}

The study consisted of a series of trials, each involving revisitation of a page selected from a long document using the scrollbar of customized interfaces. Every trial began by displaying the stimulus page on the left screen of a dual-monitor (22-inch) environment. Figure 4.1 shows the study environment.

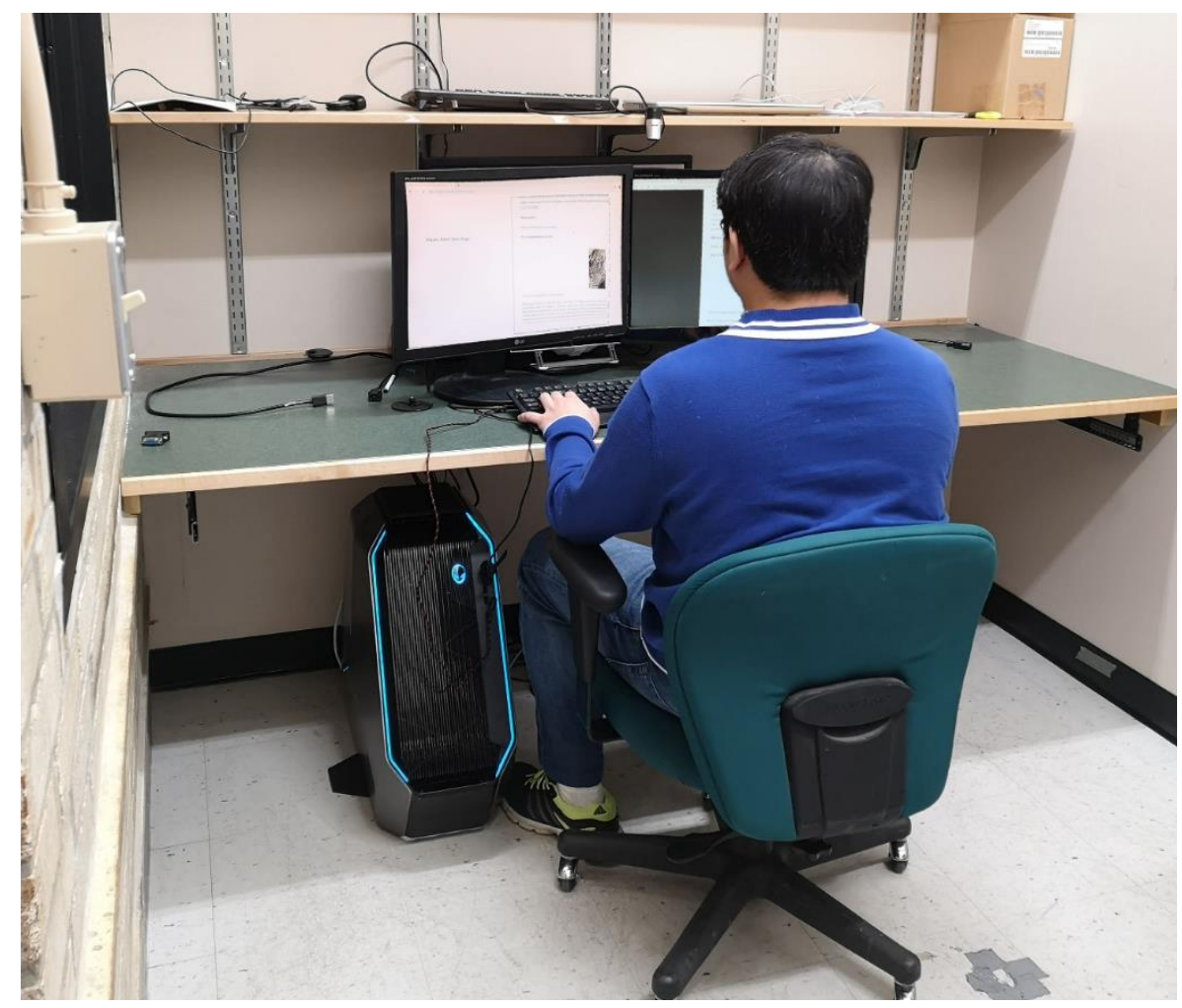

Figure 4.1. The first and second studies environment and settings

Participants then used a mouse to click, drag or scroll on the scrollbar to locate and revisit the target page using one of the five study interfaces discussed in the last chapter. The study 
interfaces ran full-screen on the right monitor. Figure 4.2 shows the dual-monitor setting used to run the experiments.

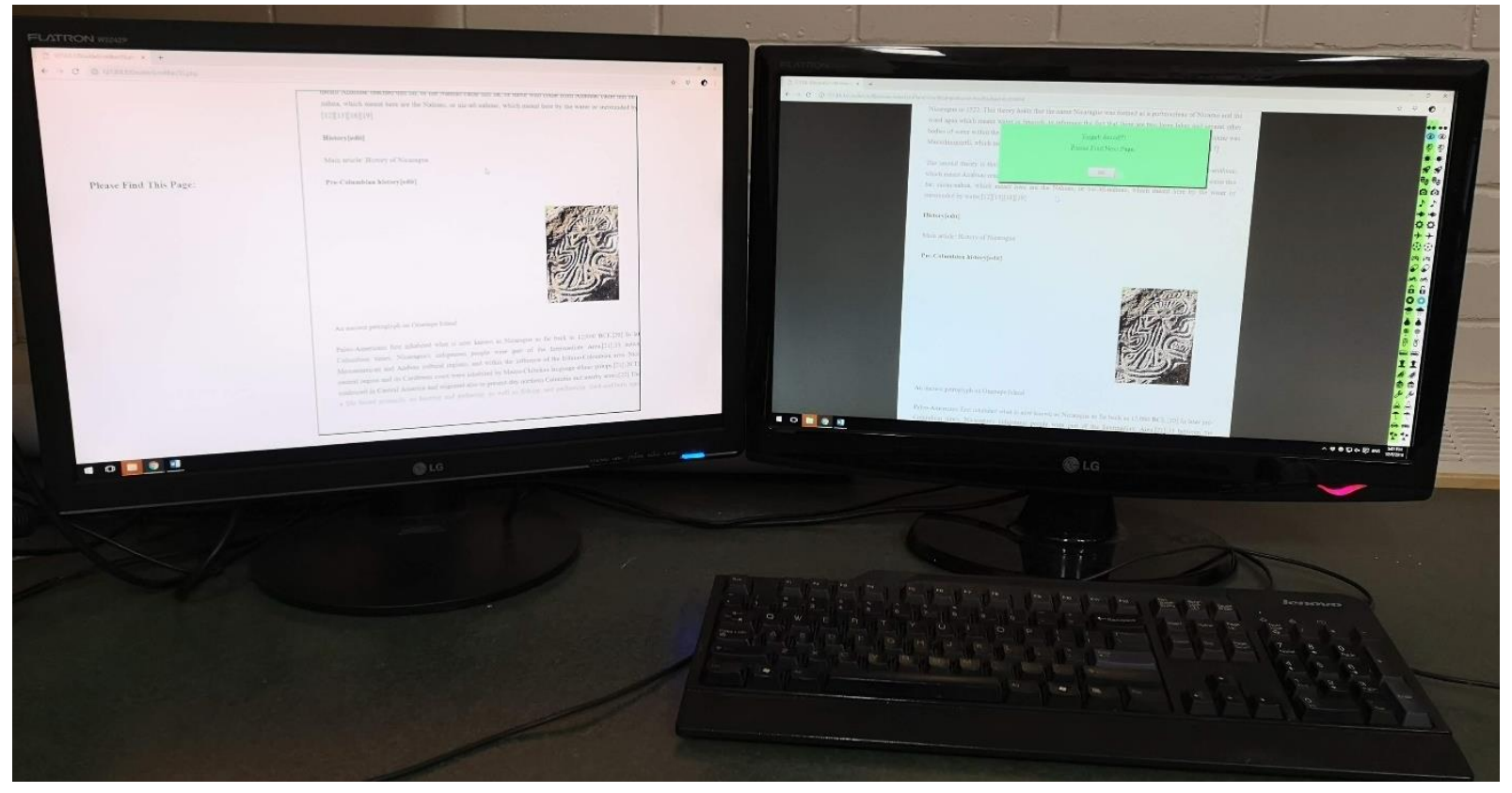

Figure 4.2. Both studies run on a dual-monitor setting. Left monitor shows the target to find, and right monitor shows the custom document viewer

A 1470-page document was used in the study for all the five interfaces. The long document was created with contents scraped from Wikipedia, containing articles on various topics, paragraphs of different lengths, and photographs of various sizes (randomly aligned to the left margin, centered, or to the right margin). Three pages were chosen manually (evenly spaced in the document) to use as stimuli with each of the five document viewer conditions; none of the target stimuli were reused in a subsequent interface.

\subsubsection{Study interfaces}

\subsubsection{The Standard Scrollbar: Single-Scrollbar with No Landmarks}

The Standard interface (see Figure 4.5) represents a single unadorned scrollbar. 
HRN, a member of the National System of Broadcasting Stations.

Cuisine[edit]

Main article: Honduran cuisine

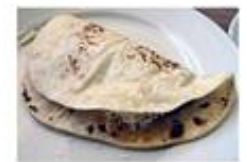

The baleada is a popular Honduran dish.

The baleada is a representative dish of the Honduran gastronomy. It consists of a flour tortilla which is folded and filled with refried beans, quesillo cheese or Parmesan cheese or sour cream. Roast meat or scrambled eggs may be added.

\section{A}

Other popular dishes include carne asada (roasted meat): chicken with rice and corn: fried fish with pickled onions; and fried or baked plantain. In the coastal areas and in the Bay Islands, seafood and meats are sometimes prepared with coconut milk.

Among the soups the Hondurans enjoy are bean soup, Sopa de mondongo (tripe soup), seafood soup, and beef soup, all of which are mixed with plaintains, cassava, cabbage, and other vegetables, and complemented by corn tortillas.

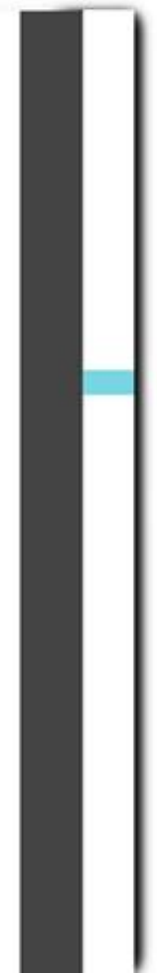

Figure 4.3. Study interface: Standard unadorned scrollbar

\subsubsection{Single-Icons: Single-Scrollbar with Icon Landmarks}

The Single-Icons interface is augmented with 30 icons (27x27px; monochrome), added vertically next to the scrollbar (see Figure 4.4). The icons are abstract, unrelated to the contents of the document, and remain the same for all documents. 


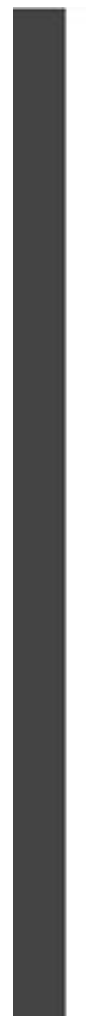

HRN, a member of the National System of Broadcasting Stations.

Cuisine[edit]

Main article: Honduran cuisine

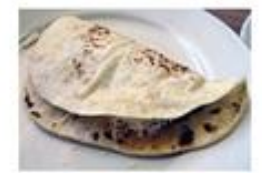

The baleada is a popular Honduran dish.

The baleada is a representative dish of the Honduran gastronomy. It consists of a flour tortilla which is folded and filled with refried beans, quesillo cheese or Parmesan cheese or sour cream. Roast meat or scrambled eggs may be added. A

Other popular dishes include carne asada (roasted meat): chicken with rice and corn: fried fish with pickled onions; and fried or baked plantain. In the coastal areas and in the Bay Islands, seafood and meats are sometimes prepared with coconut mill.

Among the soups the Hondurans enjoy are bean soup, Sopa de mondongo (tripe soup), seafood soup, and beef soup, all of which are mixed with plaintains, cassava, cabbage, and other vegetables, and complemented by corn tortillas.

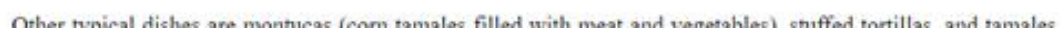

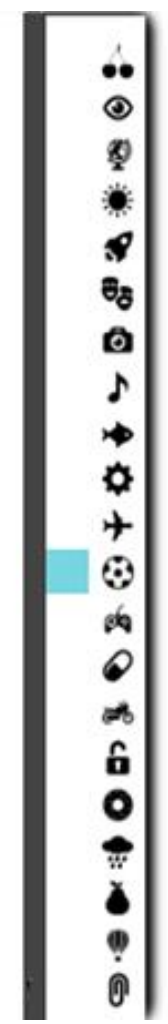

Figure 4.4. Study interface: Single-Scrollbar augmented with icons as landmarks

\subsubsection{Double-Icons: Two Scrollbars with Icon Landmarks}

Both scrollbars are augmented with 30 icons (27x27 px; monochrome, see Figure 4.5). The icons are abstract, unrelated to the contents of the document, and remain the same for all documents. 
HRN, a member of the National System of Broadcasting Stations.

Cuisine[edit]

Main article: Honduran cuisine

The baleada is a popular Honduran dish.

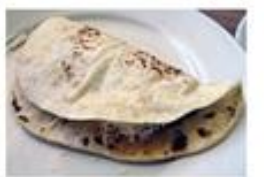

The baleada is a representative dish of the Honduran gastronomy. It consists of a flour tortilla which is folded and filled with refried beans, quesillo cheese or Parmesan cheese or sour cream. Roast meat or scrambled eggs may be added.

\section{A}

Other popular dishes include carne asada (roasted meat); chicken with rice and corn: fried fish with pickled onions; and fried or baked plantain. In the coastal areas and in the Bay Islands, seafood and meats are sometimes prepared with coconut milk.

Among the soups the Hondurans enjoy are bean soup, Sopa de mondongo (tripe soup), seafood soup, and beef soup, all of which are mixed with plaintains, cassava, cabbage, and other vegetables, and complemented by corn tortillas.

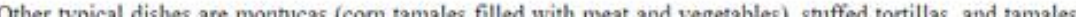

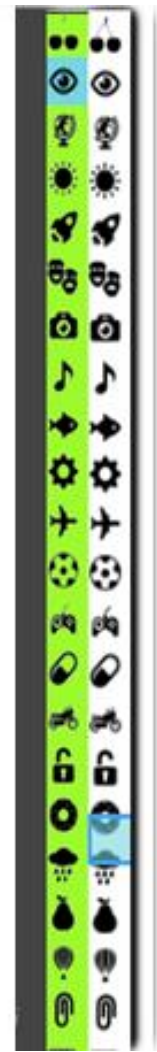

Figure 4.5. Study interface: The Double-Scrollbar with icons as landmarks

\subsubsection{Double-Letters: Two Scrollbars with Letters as Landmarks}

The Double-Letters has a similar interface to the Double-Icons, but instead of icons, 26 English letters (sorted in ascending order) and four symbols: @ , \$, \& and \# (all Arial Rounded font; 27x27 px) are used as landmarks (see Figure 4.6). 

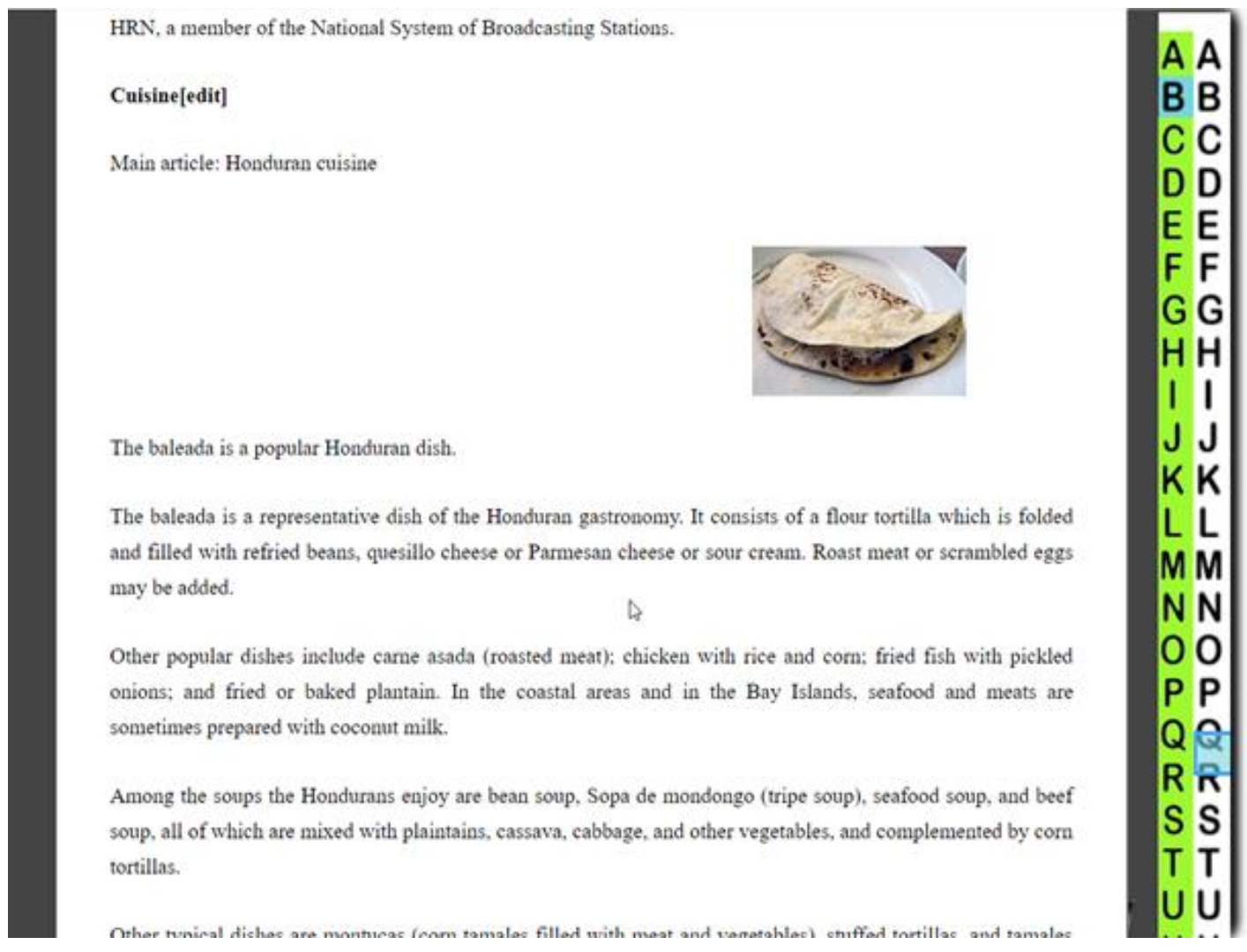

Figure 4.6. Study interface: Double-Scrollbar with letters as landmark

\subsubsection{The Double-Digits design: two scrollbars with digit landmarks}

Given that people in their daily life frequently memorized letters and are familiar with memory devices for digits (see 2.6.3), we were interested to examine the effectiveness of digits as landmarks too. The Double-Digits design has 30 digits as landmarks. Numbers start from 01 at the top and go to 30 at the bottom (see Figure 4.7). Other features are similar to Double-Icons. 


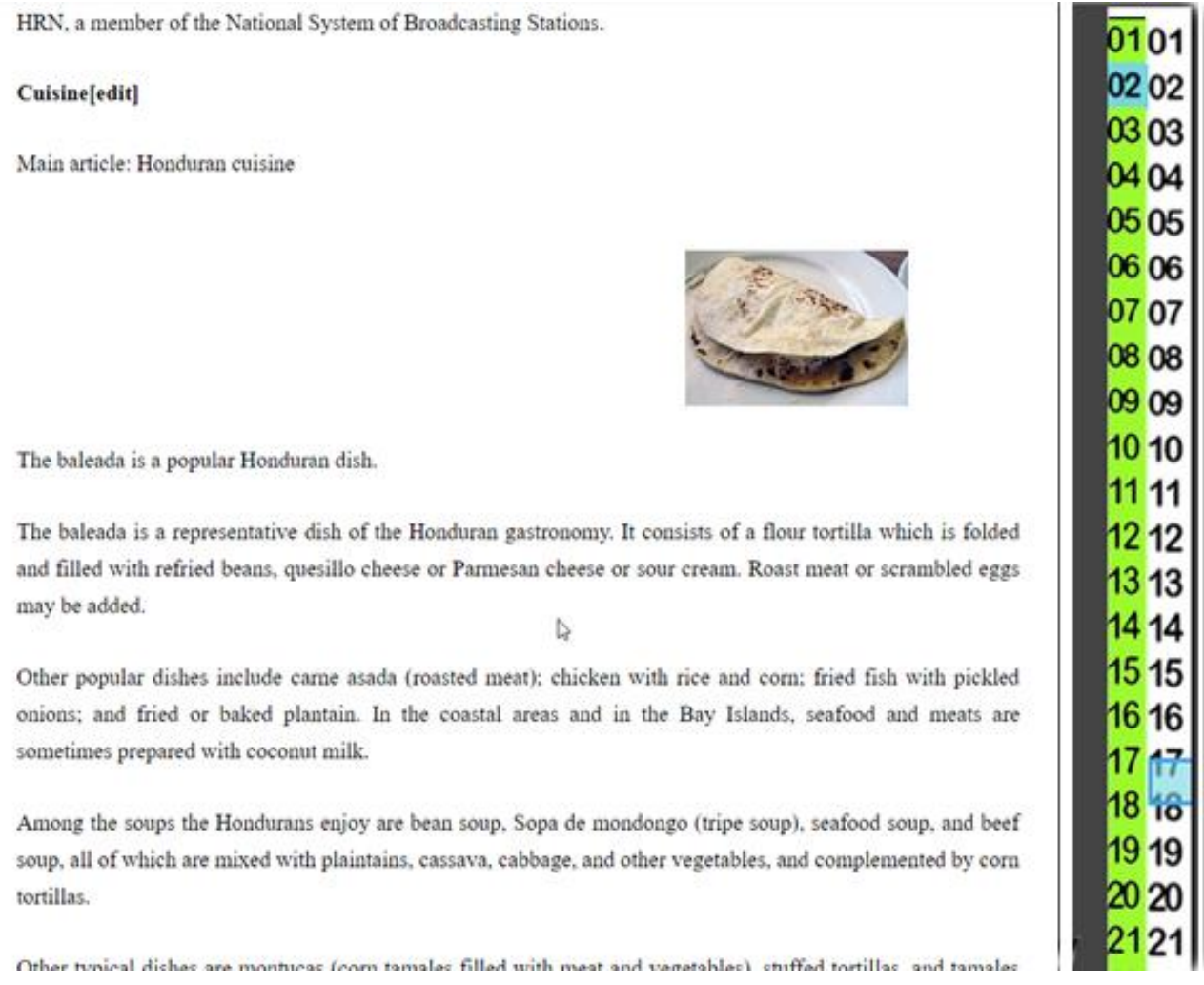

Figure 4.7. Study interface: Double-Scrollbar with digits as landmarks

\subsubsection{Procedure}

After filling out informed consent forms and demographic forms, participants were informed that the study task required a rapid revisitation of the target stimulus. The study interfaces were then demonstrated for the participants. They then went through a practice round (with two target pages) for each interface to become familiar with the systems (targets of the practice round were not repeated for the main experiment). Also, during the experiment, a set of messages were used to instruct participants on what to do after each step. Figure 4.8 shows an example of these messages. 


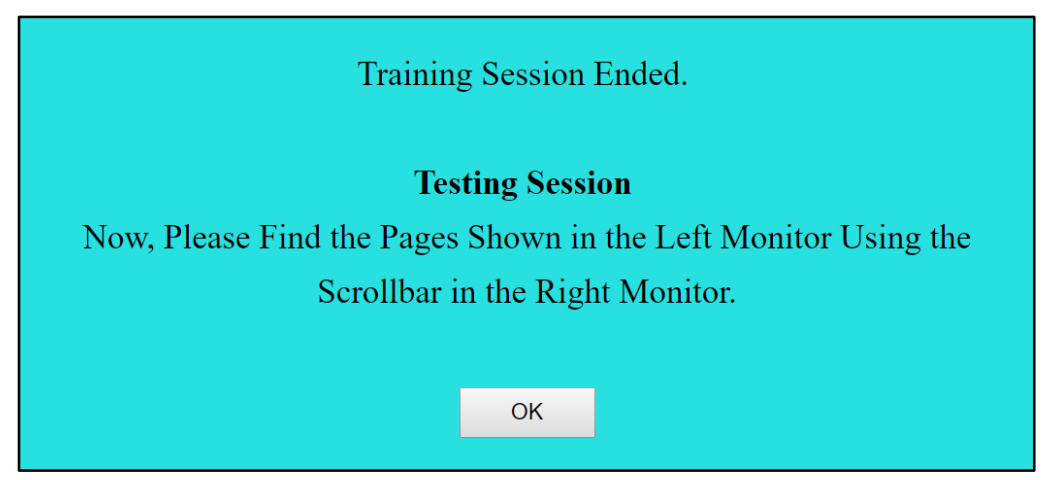

Figure 4.8. An example of the messages used to instruct participants during the study.

The study consisted of two parts for each interface: a training session and a test session. In the training session users became familiar with the target pages, resembling what happens when someone reads a document for the first time. In the test session, we measured users' ability to recall the location of target pages based on the stimuli and navigate to that page using the current scrollbar.

\subsubsection{The training session}

The training session began with a message explaining the training session to participants and asking for their confirmation to start the session. Users were shown the document using one of our custom document viewers (study interfaces) on the right-hand monitor (see Figure 4.9: a). Each page was displayed on the main window with its corresponding scroll-handle positions shown on the scrollbar (depending what type of scrollbar was used). For the whole training session, the left monitor was black with no content to show (see Figure 4.9 a).

In the training session, participants were taken to different pages of the document one page after another and were asked to skim the content and try to memorize its location in the document (see Figure 4.9 b). In this step, participants were given 15 seconds to familiarize themselves with the content of the page and its corresponding location on the scrollbar(s) and then proceed to the next target (see Figure 4.9 c). Their confirmation was required to proceed to the next page (see Figure 4.9 d). 
Confirmation in this context meant asking participants to click on the scrollbar handle/s after they had seen the target page. The reason we added this confirmation process was to engage participants with the location of the page within the scrollbar design.

The training session consisted of four blocks of training trials, meaning participants were taken to each target four times. After completion of the training session, participants were told that their learning session was over (see Figure 4.8), and the main test started.
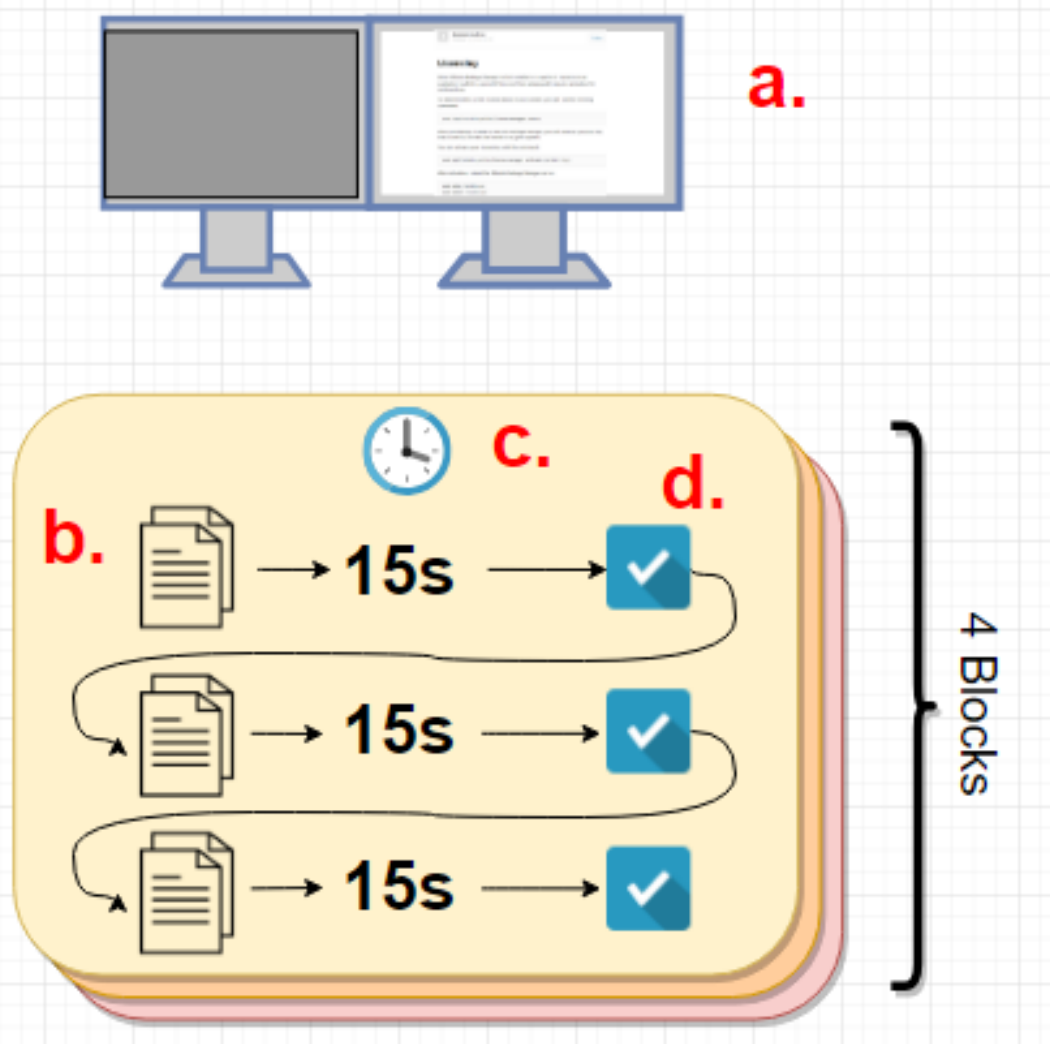

Figure 4.9. Training session process: A) shows the dual-monitor setting, with right monitor showing study interface. B) three target pages, C) maximum allowed time to visit each page, D) confirmation after each visit. Each colored rectangle represents one block of training (4 blocks for each interface) 


\subsubsection{The test session}

Followed by learning session for each condition we ran a test session on a dual-monitor setting. In this setting we presented stimulus on the left-hand monitor and study interfaces on the right-hand monitor (see Figure 4.10 a). The stimuli was one of the pages the participant had seen in the training session, randomly ordered for each block and evenly distributed throughout the document. Once the stimulus was presented on the left-hand monitor, participants had 60 seconds to find that page using one of the study interfaces on the right-hand monitor.

A correct revisitation was defined as successfully locating the target page within the given time using the available navigation techniques for the current condition. Each correct revisitation was confirmed by feedback in a green message box (See Figure 4.11 and Figure 4.10 a).

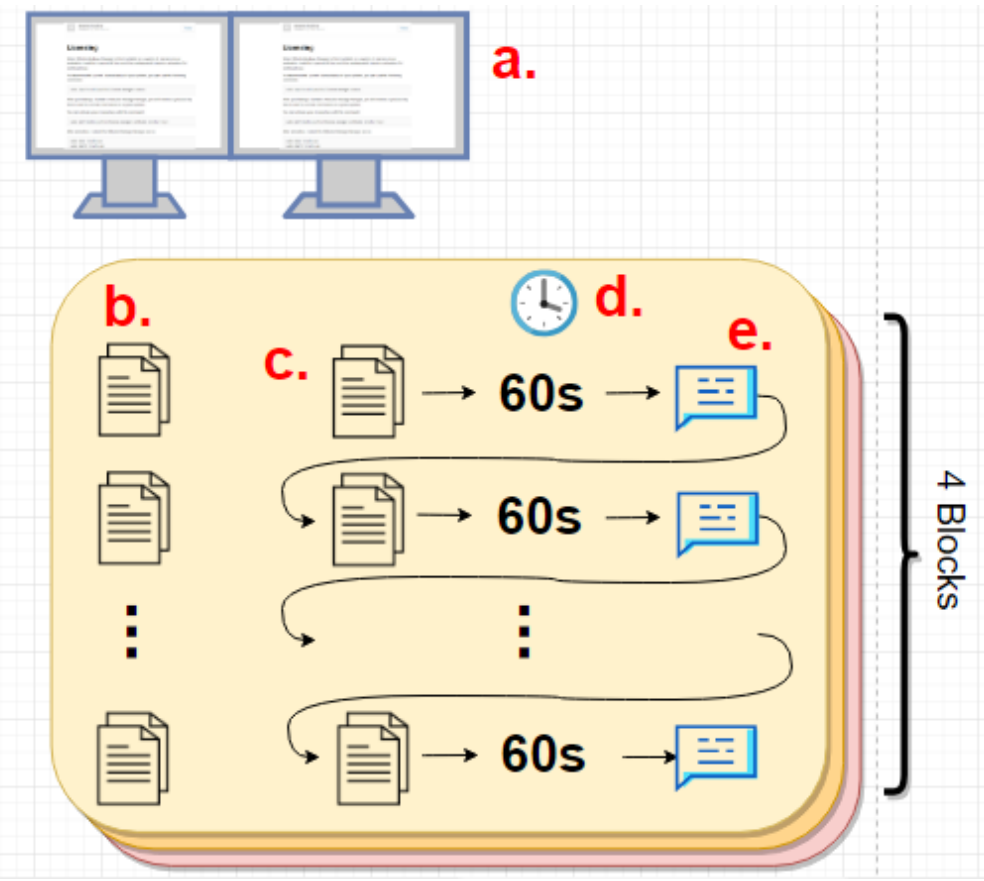

Figure 4.10. Test session: A) shows the dual-monitor setting, with right monitor showing study interfaces and left monitor study stimuli; B) three screenshots of target pages shown as stimuli on left monitor; C) target pages in the document shown on right monitor; D) maximum allowed time to visit each page; E) confirmation after each revisit. Each colored rectangle shows one block of the test session (4 blocks for each interface) 
Unsuccessful revisitations were defined as a failure to find a target in the given time and were recorded as Timeouts in the results below. Timeouts were indicated to users in a red message box (see Figure 4.12 and Figure 4.10 a), and the actual location of the page on the scrollbar was highlighted. Software recorded the time for each trial (successful or unsuccessful), activities on the scrollbar(s), and data describing every selection. The time for each trial began when the stimulus was shown on the left monitor and ended when the participant either found the target page or failed to find it in the given time (Timeout).

Target: found!!!

Please Find Next Page.

OK

Figure 4.11. The feedback message for a successful revisitation.

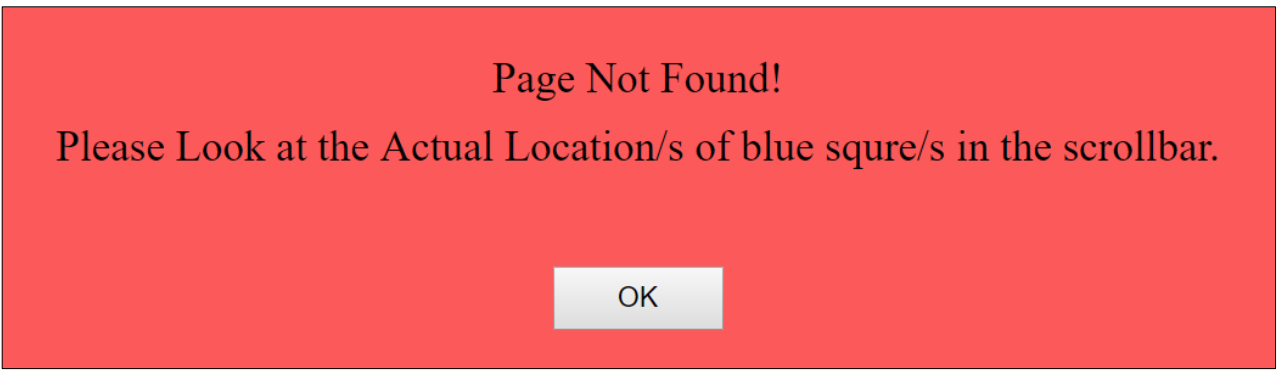

Figure 4.12. The feedback message for an unsuccessful revisitation. 


\subsubsection{Study design}

\subsubsection{Dependent variables}

Dependent variable for this experiment were measured and reported as follows:

Mean Task Completion Time: Mean task completion time is defined as the time users spent (in milliseconds) to do a successful revisitation and started when users were shown the stimuli on the left-hand monitor. We calculated mean trial completion time for each block by averaging the total trial time all participants spent to visit the targets of each block.

Scrollbar interactions: defined as all interaction users made to make a revisitation (successful or unsuccessful), including the number of clicks on both scrollbars, scrolling using the mouse wheel, and dragging the scrollbar handle for each trial.

Number of time-outs: defined as the number of unsuccessful revisitations (failed to be completed in the given time).

These measures were logged by the experiment software.

\subsubsection{Independent variables}

Independent variable for this study were the following:

Interface type: We compared three different interfaces: the Standard scrollbar, the SingleScrollbar augmented with icons as landmark, and the Double-Scrollbar augmented with three type of landmarks.

Landmark type: We were also interested in studying the impact of landmark type on users' performance; therefore, we examined letters, digits, and icons as three types of landmarks added to the Double-Scrollbar. 


\subsubsection{Hypotheses}

The purpose of the study was to explore the following hypotheses:

H1. Trial completion time for the standard scrollbar will be higher than all other interfaces

H2. The number of scrollbar interactions for the standard scrollbar will be higher than all other interfaces

H3. Trial completion time for the Single-Icons scrollbar will be lower than the standard scrollbar and higher than the Double-Scrollbar designs

H4. The number of scrollbar interactions for the Single-Icons scrollbar will be lower than the standard scrollbar and higher than the Double-Scrollbar designs

\subsubsection{Participants}

Five participants (two females), ages (24-31), from the University of Saskatchewan volunteered for this study. The study lasted 90 minutes on average for each participant.

\subsubsection{Apparatus}

The experiment was conducted on a desktop computer running Windows 10 (8 GB RAM, Intel Core i7-2600 CPU and NVIDIA GeForce GT 520 VGA), with two 22-inch (1920x1080 resolution) monitors placed alongside. The software was written in HTML and JavaScript, running on Google Chrome browser. Input was received through a standard optical mouse.

\subsubsection{Results}

In this section, we present the results of the preliminary study, comparing standard scrollbar with other landmark-augmented interfaces and report our dependent variables as the number of interaction and trial completion time. 
In both studies, we report the effect size for significant RM-ANOVA results as general etasquared: $\eta^{2}$ (considering 0.01 small, 0.06 medium, and >0.14 large [19]). Also, results obtained from all participants for all metrics fell within two standard deviations from the mean, so no data were removed as outliers.

\subsubsection{Trial completion time}

As mentioned in 4.1.4.1, mean trial completion time is defined as the time users spent (in milliseconds) to do a successful revisitation and started when users were shown the stimuli on the left-hand monitor. Mean trial completion times across blocks are summarized in Figure 4.13 for the five conditions.

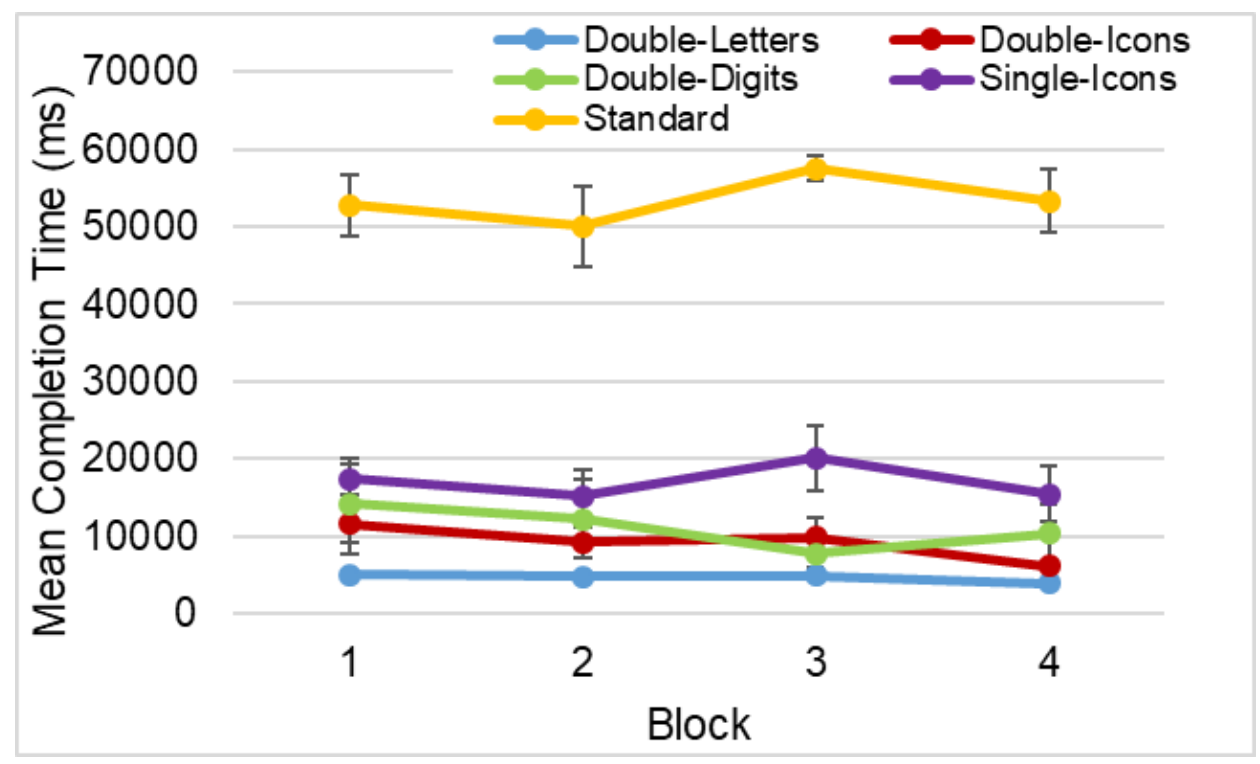

Figure 4.13. Trial completion time ( \pm s.e.) by block.

RM-ANOVA showed a significant main effect of condition $\left(F_{4,16}=33.20, p<.001, \eta^{2}=0.84\right)$, but no effect of block, and no condition $\times$ block interaction (all $F<1.22$ and $p>.35$ ).

Bonferroni-corrected post-hoc t-tests showed that the Standard condition was slower than all other interfaces (all $p<.001$ ) by a large margin, with a mean trial completion time $53407 \mathrm{~ms}$ (s.d. 15144ms). Therefore, we accept H1. With trial time less than 9127ms (s.d. 9234), Double-Icons 
and Double-Letters performed significantly better than Single-Icons (all $p<.01$ ) but there was no difference between themselves $(p=.53)$.

\subsubsection{Scrollbar interactions}

As mentioned in 4.1.4.1, scrollbar interactions referred to users' interactions to make a revisitation, including the number of clicks on the scrollbars, scrolling using the mouse wheel, and dragging the scrollbar handle. Figure 4.14 presents the analysis of the scrollbar interactions (e.g., number of clicking on both scrollbars, scrolling using the mouse wheel, or dragging scrollbar nob) per trial.

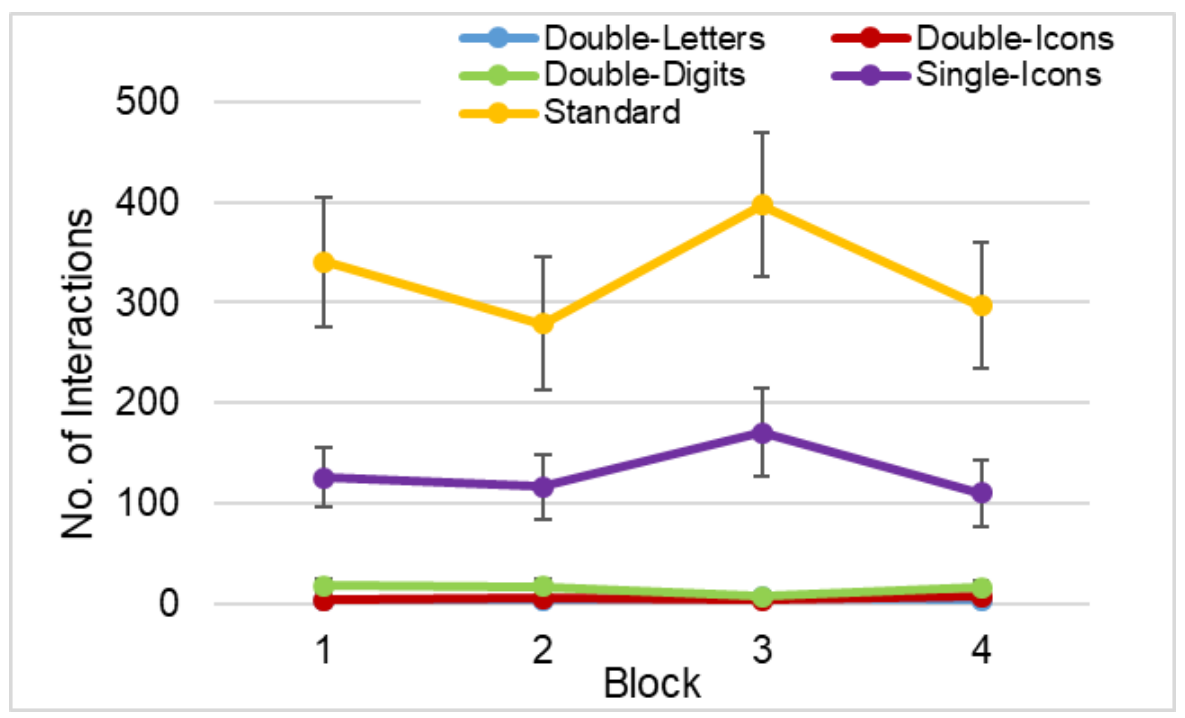

Figure 4.14. Total scrollbar interactions by block.

ANOVA showed a significant main effect of condition $\left(F_{4,16}=10.21, p<.001, \eta^{2}=0.67\right)$ and block $\left(F_{3,12}=4.23, p=.03, \eta^{2}=0.02\right)$. There was, however, no condition $\times$ block interaction $\left(F_{12,48}=1.08\right.$, $p=.39$ ). Post-hoc analysis showed that both Standard and Single-Icons conditions had a large number of scrollbar interactions (more than 130/trial, s.d. 135) compared to the three two-level conditions (less than 14.5/trial, s.d. 23.6), all $p<.001$. We therefore, accept H4. The Standard scrollbar had twice the number of the Single-Icons design $(p<.001)$ with mean interactions 340/trial (s.d. 278). As a result, we accept $\mathbf{H 2}$. 


\subsubsection{Discussion}

Considering the significant difference between the Standard scrollbar and the landmarked designs (in terms of trial completion time and the number of interactions), we concluded that the limitations of the Standard design for revisitation in long documents was clear. We believe the adjacency of the scrollbar handle to artificial landmarks on the augmented scrollbars provided reference points for users to form stronger spatial memory and find target pages faster with fewer scrollbar interactions. In contrast, the lack of landmarks on the Standard scrollbar led to inaccurate estimations of the position of scrollbar handle, leading to more scrollbar interactions and longer task completion times.

Figure 4.13 and Figure 4.14 also show that revisitation using the single-level design required longer time and more scrollbar interactions. Our explanation is that when the document is long, one level of landmarks does not provide enough precision in the selection, which forces users to make time-consuming corrections to find the right target page.

Therefore, we decided to exclude the standard condition from our main study, and instead compare the landmarked interfaces in more detail.

\subsection{STUDY 2: LANDMARK-BASED REVISITATION IN LARGE DOCUMENTS}

The goal of the main study was to find out whether there are significant differences in revisitation performance between the Single-Icon design and different versions of the Double-Scrollbar design. Similar to the preliminary study, we measured revisitation performance by recording users' revisitation time and the number of interactions they made for each successful or failed revisitation. We tested the following interfaces:

- Single-Icons: Single-Scrollbar with icon landmarks

- Double-Icons: Double-Scrollbar with icon landmarks 
- Double-Letters: Double-Scrollbar with letter landmarks

- Double-Digits: Double-Scrollbar with digit landmarks

\subsubsection{Method}

The second study used the same method as Study 1, but with some adjustments: First, the number of conditions decreased to four as we removed the Standard scrollbar. Second, the length of the long document was also altered from 1470 pages to 900, to make the number of pages and blocks even for all of the designs (30 blocks, each with 30 pages); 900 pages is still considerably larger than the 42-page document used in earlier work [82]. Third, the number of target stimuli was increased to six pages and each page of these pages were visited twice in the training phase. Among the six stimuli pages, two were chosen with no images in them (only text was present). Last, the six target stimuli were grouped into two categories based on their proximity to landmarks: for the "on landmark" group, the target page's corresponding scrollbar handle were exactly aligned with the landmark (see Figure 4.15.a and Figure 4.15.c); for the "near landmark" group, the corresponding scrollbar handles were not aligned with landmarks ( Figure 4.15.b and Figure 4.15.d).

a.

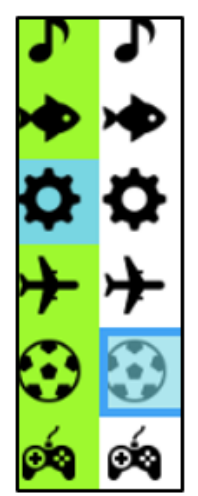

b.

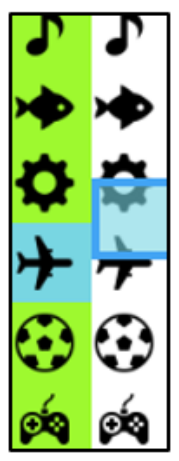

c.

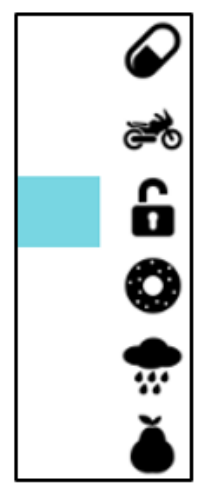

d.

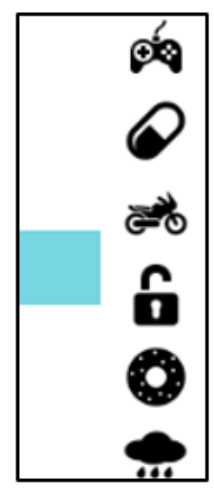

Figure 4.15. On landmark (a. and c.) and near landmark (b. and d.) in Double-Scrollbar and Single-Scrollbar designs 
Other aspects of the method, procedure, and apparatus were the same as Study 1, except that after completing each condition, participants also completed a NASA-TLX [36] subjective workload questionnaire, and at the end of each study, participants provided subjective preference responses through a questionnaire.

\subsubsection{Experimental conditions, Study interfaces, Procedure, and Apparatus}

Experimental conditions in Study 2 operated as described above, and included the Single-Icon, Double-Icon, Double-Letters, and Double-Digits designs. The procedure did not change for the second experiment and was as explained in section 4.1.3. The apparatus remained the same, as explained in section 4.1.2.

\subsubsection{Study design}

Dependent variables used in the first study were also used here: revisitation time, number of navigation actions, and number of timeouts (see section 4.1.4.1). In addition, we measured:

- Error distance on first click: the number of pages between the position of a user's first click and the target page for each trial.

- Fit to the power law of practice: Regression analysis of task completion time across blocks to examine if any learning happened

- Perceived workload: using the NASA TLX questionnaire

- Overall preference: using the subjective preference questionnaire

For independent variables, we also used interface type and landmark type (see 4.1.4.2), but also added the following exploratory independent variables to investigate differences in task completion time and number of interactions. 
- Page content (text-only or text plus picture): we analyzed user performance in terms of time and number of interactions when a target page had only text or text and pictures.

- Proximity to landmark: we analyzed performance based on whether the scrollbar handle was "on landmark" or "near landmark" (see section 4.2.4.6).

Therefore, the study used a repeated-measures design with two main factors: type of interface (Single or Double scrollbar) and type of landmark (icons, letters, and digits). However, the design was not fully crossed, so we used a single-factor analysis based on the combination of the two main factors. Other independent variables (distance, content, proximity) were used for exploratory analyses.

\subsubsection{Hypotheses}

The study explored the following hypotheses:

H1. Trial completion time for Single-Icons will be higher than all other interfaces

H2. The number of scrollbar interactions for the Single-Icons design will be higher than all other interfaces

H3. Users' revisitation time will decrease for each block satisfying the power law of practice [59], indicating that learning is occurring.

Also, additional analysis on new independent variables, introduced in 4.2.2, will be done as follow ups to provide support for main findings. 

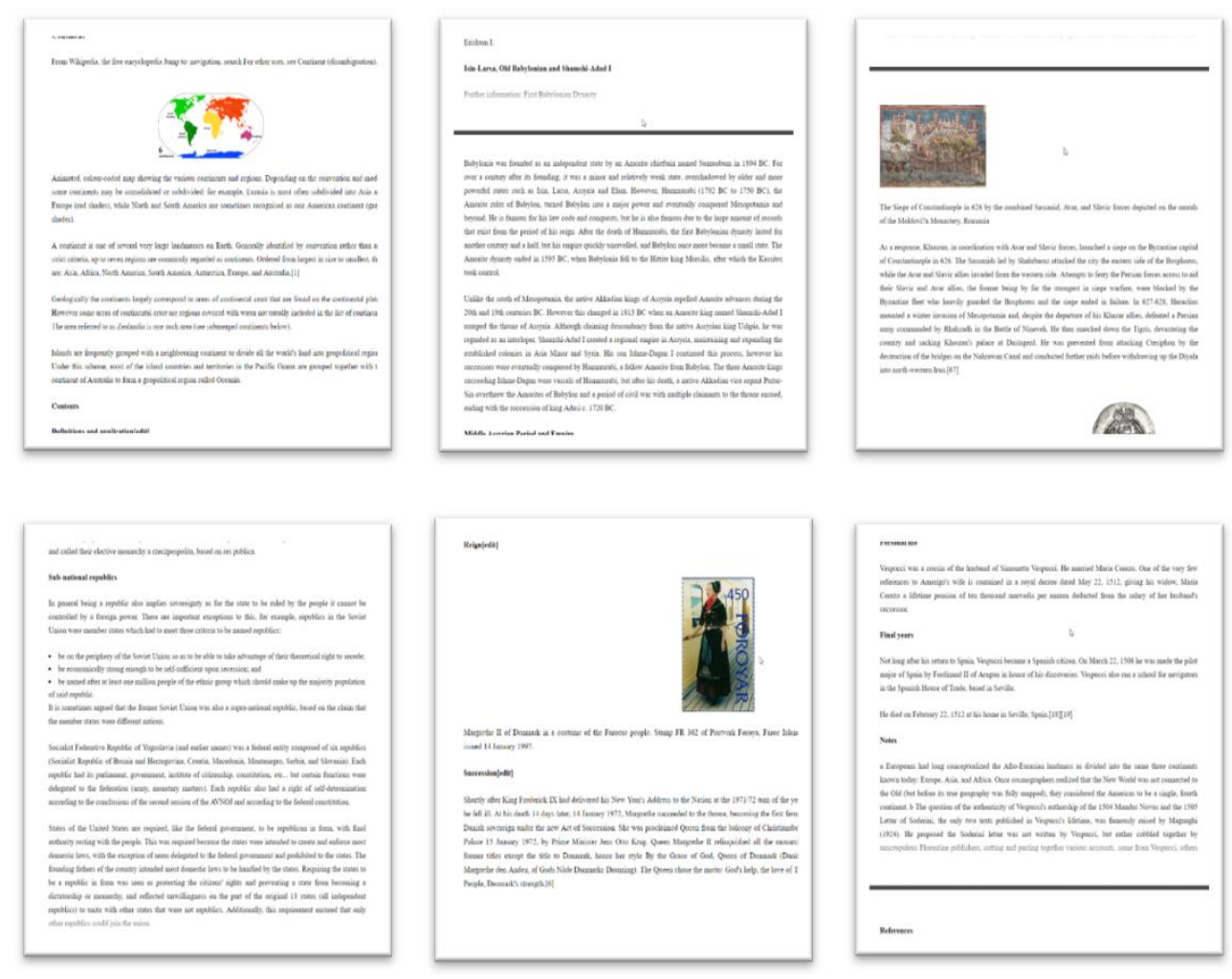

Figure 4.16. Examples of pages used as targets.

\subsubsection{Participants}

Seventeen new participants (all students) who were not part of Study 1 were recruited from the University of Saskatchewan by posting an ad on the university portal. Due to a technical issue, one participant could not finish the experiment, leaving 16 participants (nine males, seven female), ages 19-48 (mean 27.63) who completed the study. The study lasted 60 minutes for each participant and they were given a $\$ 10$ honorarium. All of the participants were familiar with document viewers: $37.5 \%$ used document viewers between one to 10 hours per week, $18.75 \%$ between 11 to 20 hours, and 43.75\% more than 20 hours per week. Questionnaires also revealed that Adobe Reader was the most popular document reader among participants, listed by 13 participants as their primary document reader. Four participants stated that they primarily used Foxit Reader, and three participants used their browser's built-it PDF reader. 


\subsubsection{Results}

Greenhouse-Geisser adjustments were performed on the results of this study (fractional degrees of freedom), where ANOVA's sphericity assumption is violated (Mauchley's test). Analysis showed that results obtained from all participants for all metrics fall within two standard deviation which means there were no outliers.

\subsubsection{Trial Completion Time}

As mentioned in section 4.1.4.1, trial completion time was the time users took to do a successful revisitation (a trial started when users were shown the stimuli on the left-hand monitor, and ended with a successful revisitation or a timeout). Figure 4.17 summarizes mean trial completion times across blocks for four conditions.

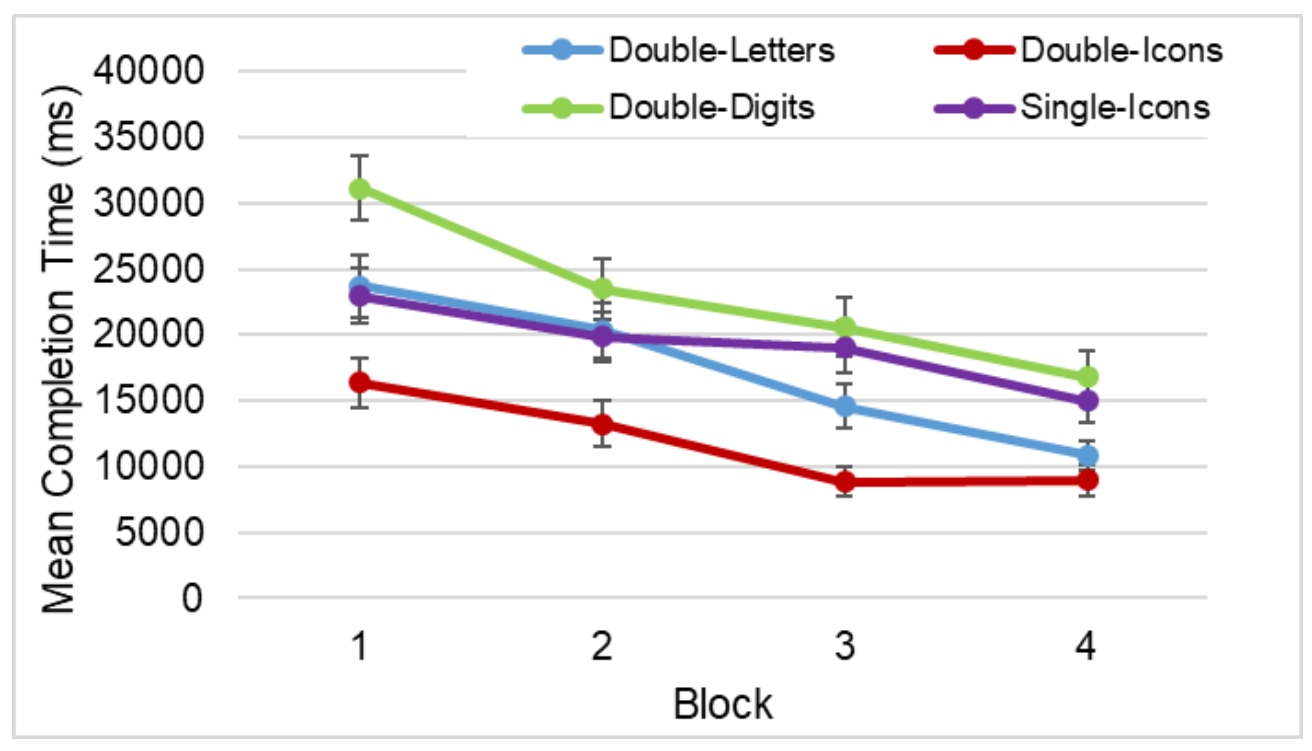

Figure 4.17. Mean completion time ( \pm s.e.) by condition and block.

RM-ANOVA showed a significant main effect of condition $\left(F_{3,45}=5.31, p=.003, \eta^{2}=0.09\right)$ with mean trial completion time 11859ms (s.d. 15090ms) for Double-Icons, 17360ms (s.d. 18877ms) for Double-Letters, $19213 \mathrm{~ms}$ (s.d. 18731ms) for Single-Icons, and 23006ms (s.d. 22783ms) for Double-Digits. Post-hoc pairwise t-tests (Bonferroni-corrected) showed that Double-Icons condition was faster than all other interfaces (all $p<.001$ ). Single-Icons and Double-Letters were 
faster than Double-Digits (all $p<0.04$ ), but showed no difference between themselves. Therefore, H1 is rejected.

As shown in Figure 4.13, completion times decreased across block $\left(F_{1.59,23.85}=30.88, p<.001\right.$, $\eta^{2}=0.09$ ), and the skill development follows a power law function [59], as expected. Therefore, $\mathbf{H 3}$ is accepted. There was, however, no significant condition $\times$ block interaction $\left(F_{3.96,59.4}=1.56, p=.2\right)$.

\subsubsection{Scrollbar interactions}

Analysis of the total number of scrollbar interactions (e.g., clicking on the trough, using the scroll wheel, or dragging the scroll handle) per trial is presented in Figure 4.18. RM-ANOVA showed a significant main effect of condition $\left(F_{1.92,28.8}=11.86, p<.001, \eta^{2}=0.21\right)$, with mean interactions 14.7/trial (s.d. 50.0) for Double-Icons, 38.4/trial (s.d. 112.4) for Double-Letters, 45.7/trial (s.d. 123.9) for Double-Digits, and 125.6/trial (s.d. 167.4) for Single-Icons.

As shown in Figure 4.18, although mean scrollbar interactions/trial decreased slightly over time, especially for the three two columns of landmarks conditions, there was no significant effect of block $\left(F_{1.44,21.6}=2.72, p=.1\right)$, and no condition $\times$ block interaction $\left(F_{3.6,54.0}=1.24, p=.31\right)$.

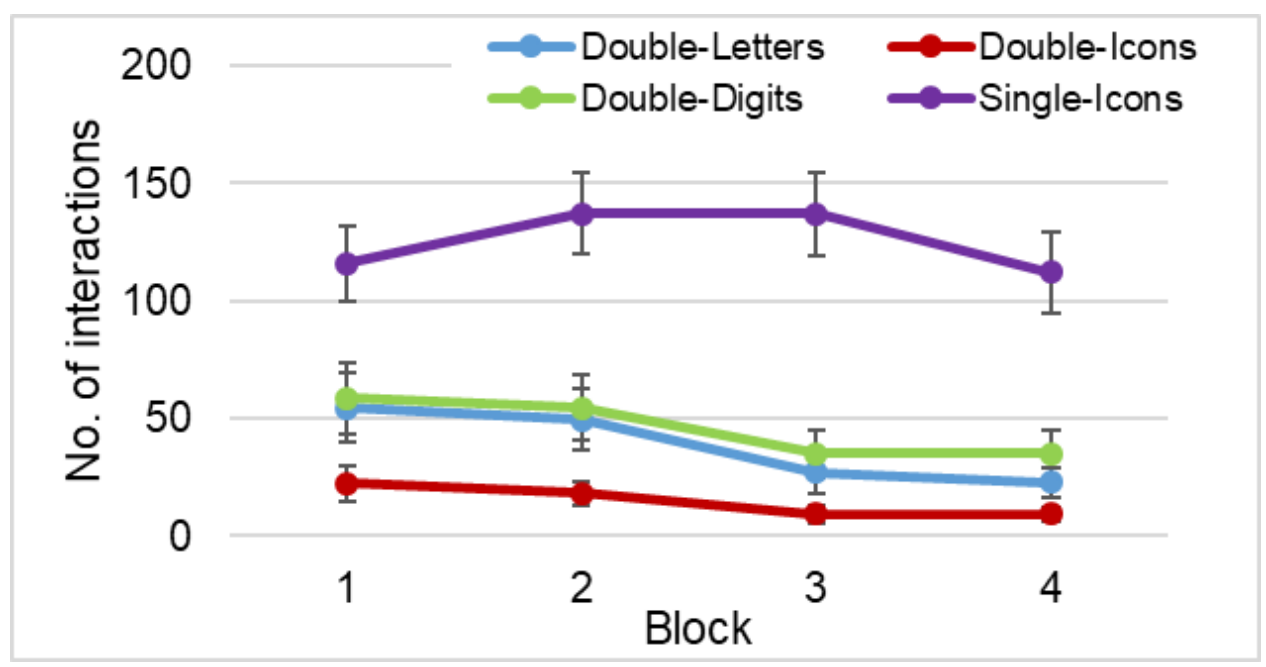

Figure 4.18. Scrollbar interaction ( \pm s.e.) by condition and block. 
Bonferroni-corrected post-hoc t-tests showed that the Double-Icons condition and Single-Icons had the least (all $p<.04$ ) and the highest (all $p<.001$ ) number of scrollbar interactions per trial respectively among all the conditions. We therefore, accept H2. There was no difference in terms of scrollbar interactions between Double-Letters and Double-Digits.

In the study, a total of 86,219 interactions were made with the scrollbar, comprised of $94.5 \%$ scrolling using the mouse wheel, $4.9 \%$ clicking on the scrollbars, and $0.5 \%$ dragging the scrollbar handle. We analyzed the scrolling and clicking activity separately which is presented in Figure 4.19.

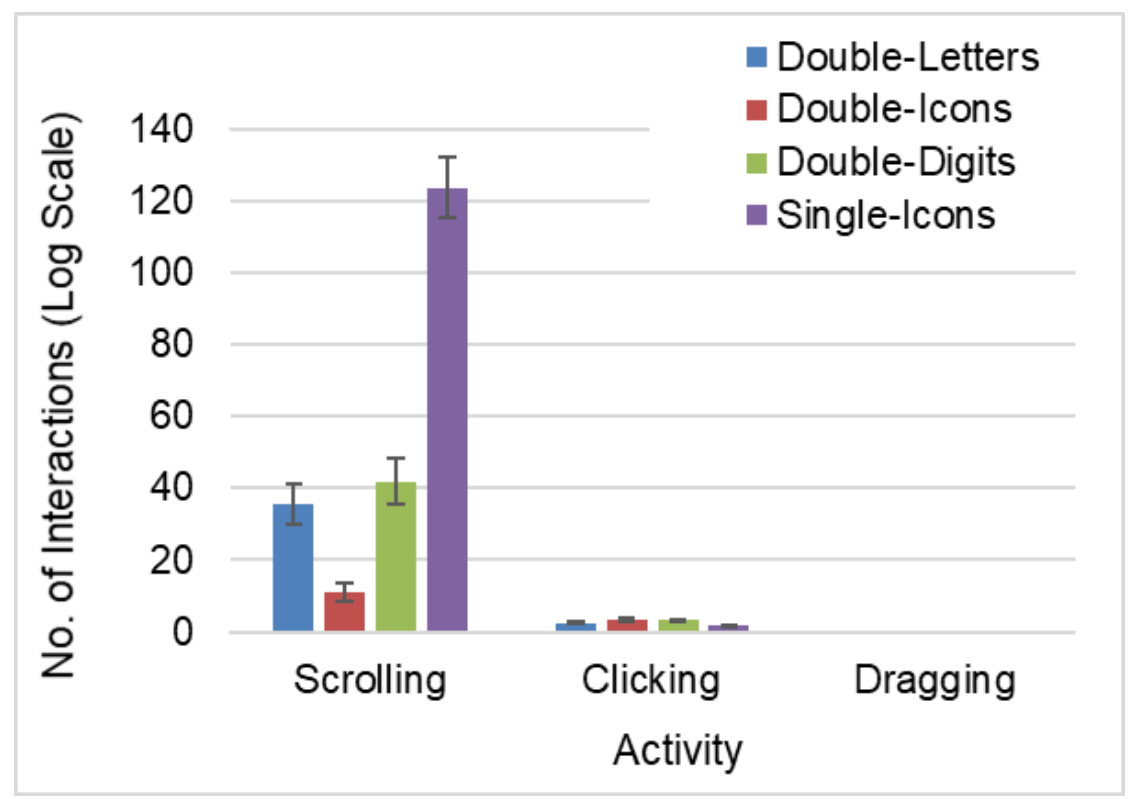

Figure 4.19. Scrollbar interactions $( \pm$ s.e. $)$ by activity.

For scrolling activity, ANOVA showed a significant main effect of condition $\left(F_{1.92,28.8}=11.78\right.$, $\left.p<.001, \eta^{2}=0.21\right)$. However, there was no significant main effect of condition $\left(F_{1.35,20.25}=1.82\right.$, $p=.19)$ for clicking activity. Post-hoc analysis showed that mean the number of scrolling was least with the Double-Icons condition (11.2/trial, s.d. 49.4) and most with the Single-Icons condition (123. 7/trial, s.d. 167.0), all $p<.03$. A substantial amount of the mouse wheel scrolling for SingleIcons compared to other interfaces can be attributed to this interface's inefficiency in obtaining a precise selection. Our explanation is that in Single-Icons design presence of icons helps with the 
formation of spatial memory, therefore, the user can reach the vicinity of the target by recalling the right icon (and performing the least number of clicks as shown in Figure 4.19). However, after the initial click, any slight movement of scrollbar knob can take user many pages away from the target, forcing users to rely more on the scrolling using the mouse wheel to find the target page. Relying on scrolling using mouse wheel (as shown in Figure 4.19) can increase the number of interactions and mean completion time (as shown in Figure 4.18).

\subsubsection{Time-out analysis}

There was a time-out period (60 seconds) for each trial. We analyzed the number of times participants failed to revisit a target page (see Figure 4.20).

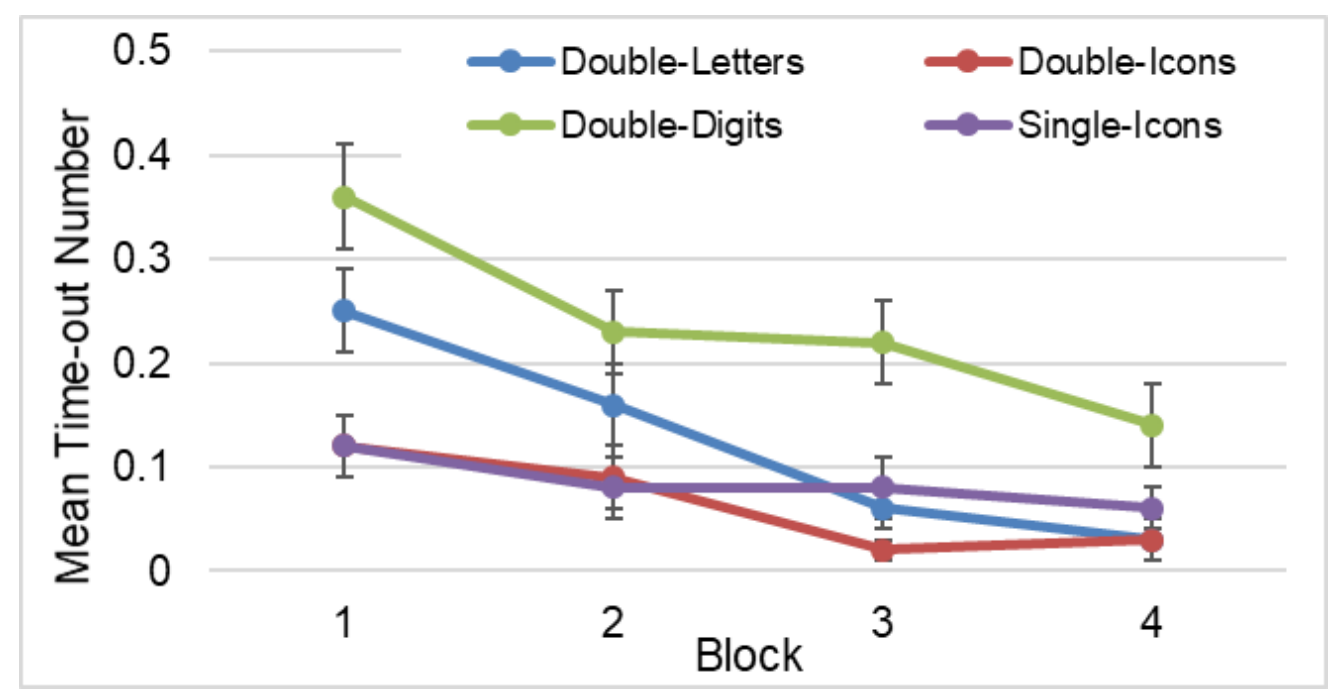

Figure 4.20. Mean number of time-out ( \pm s.e.) by block.

RM-ANOVA showed a significant main effect of condition $\left(F_{2.01,30.15}=5.05, p<.001, \eta^{2}=0.11\right)$ with mean time-outs 0.07/trial (s.d. 0.25) for Double-Icons, 0.09/trial (s.d. 0.28) for Single-Icons, 0.12/trial (s.d. 0.33) for Double-Letters, and 0.24/trial (s.d. 0.43) for Double-Digits. The number of time-outs decreased across blocks as anticipated yielding significant effect of block $\left(F_{3,45}=14.12, \quad p^{<.001,} \eta^{2}=0.08\right)$. There was no significant condition $\times$ block interaction $\left(F_{3.69,55.35}=1.74, p=.09\right)$. Post-hoc t-test showed that Double-Digits had the greatest number of 
time-outs per trial (all $p<.001$ ). However, there was no difference among the other three conditions (all $p>.1)$.

We also analyzed the time-outs by page content (text only, or text + pictures). We did not find any significant main effect of content and no content $\times$ condition interaction (all $F>1.5$ and $p>.18$ ).

\subsubsection{Analysis of the error distance in the first click}

We analyzed the mean error distance of the participant's first selection in the scrollbar, across blocks (see Figure 4.21). To calculate error distance, we counted the number of pages between the position of a user's first click and the target page for each trial and divided it to number of interactions of that trial. If the selected page was the same as target page (a successful revisitation) distance was equal to zero. Then we multiplied the number of pages by the length of each page (1400 pixels) to calculate the distance in pixels. Finally, we calculated the mean distance for each block by dividing the average distance for all users for all targets by the total number of trials in that block.

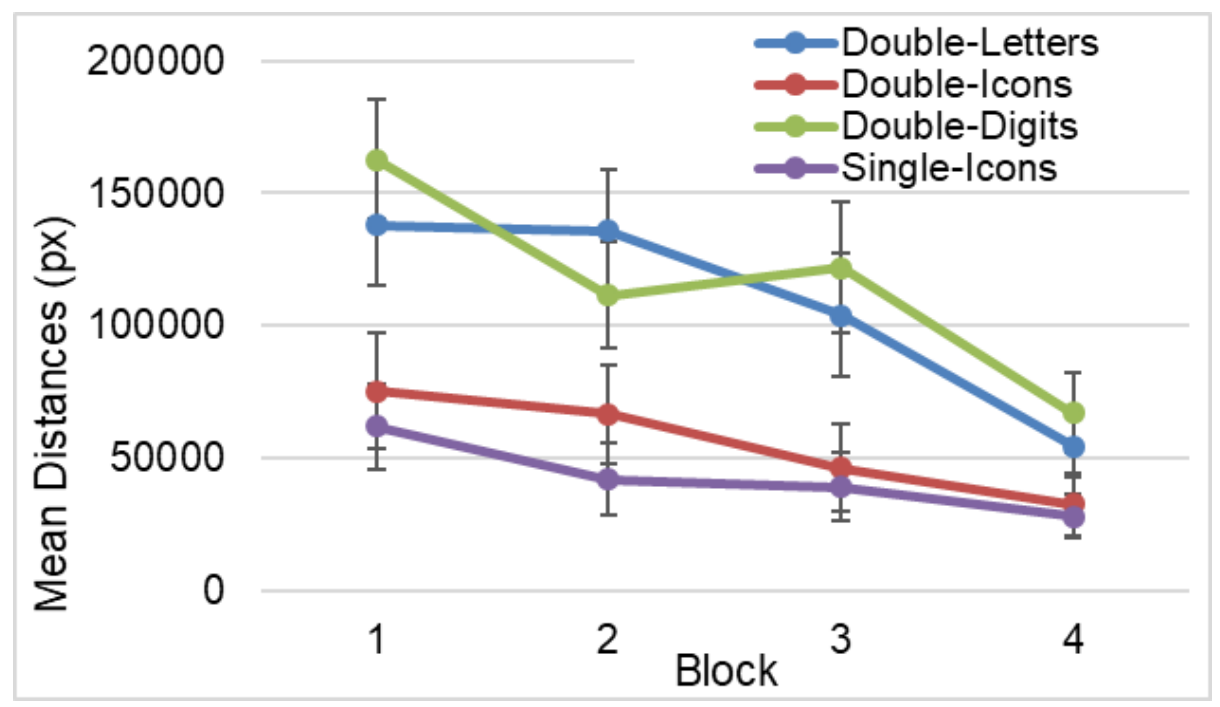

Figure 4.21. Mean distances ( \pm s.e.) of scrollbar interactions.

ANOVA analysis showed a significant main effect of condition $\left(F_{1.83,27.45}=3.59, p=.045, \eta^{2}=0.08\right)$.

As seen in Figure 4.21, mean distances decreased across block $\left(F_{3,45}=8.28, p<.001, \eta^{2}=0.05\right)$, but 
there was no condition $\times$ block interaction $\left(F_{4.59,68.85}=0.99, p=.43\right)$. The decrease in mean distance for all landmark augmented interfaces can be another indication that users relied more on their memory to find targets rather purely depending on the visual search.

Post-hoc analysis showed that both Double-Icons (55247px/trial, s.d. 172499) and Single-Icons (42846px/trial, s.d. 127380) performed better (all $p<.001$ ) than other two interfaces, but no difference between themselves $(p=1)$. The reason for Single-Icons having the lowest mean distance is that in this interface user had the greatest number of interactions (presented in Figure 4.14) which lowered mean distances for this interface.

\subsubsection{Analyses of targets by page content}

Among the six targets, four had distinctive images on them, and the other two had only text in the page. Figure 4.22 summarizes the analysis of trial completion time by page content for four conditions.

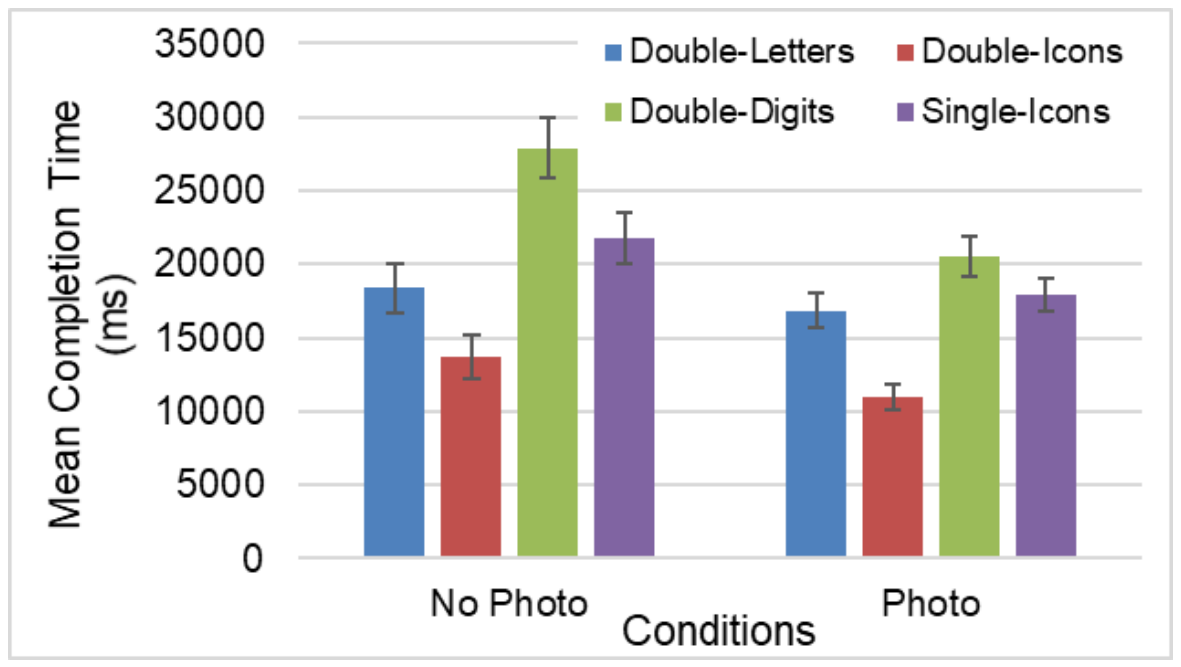

Figure 4.22. Analyses of mean completion time based on page content.

RM-ANOVA showed a significant main effect of content $\left(F_{1,15}=13.64, p=.002, \eta^{2}=0.02\right)$ and condition $\left(F_{1.89,35.28}=5.84, p=.008, \eta^{2}=0.08\right)$ with mean trial completion time 16568.35 (s.d. $18819 \mathrm{~ms}$ ) for photo, $20442 \mathrm{~ms}$ (s.d. 20476ms) for no photo. However, there was no content $\times$ condition interaction $\left(F_{3,45}=1.93, p=.14\right)$. Post-hoc pairwise t-tests showed that Double-Icons 
performed best among four (all $p<.002$ ) where targets had a photo (10930ms, s.d. 14084ms). In case of targets with no photo, Double-Icons performed faster than Single-Icons and Double-Digits (all $p<.01)$.

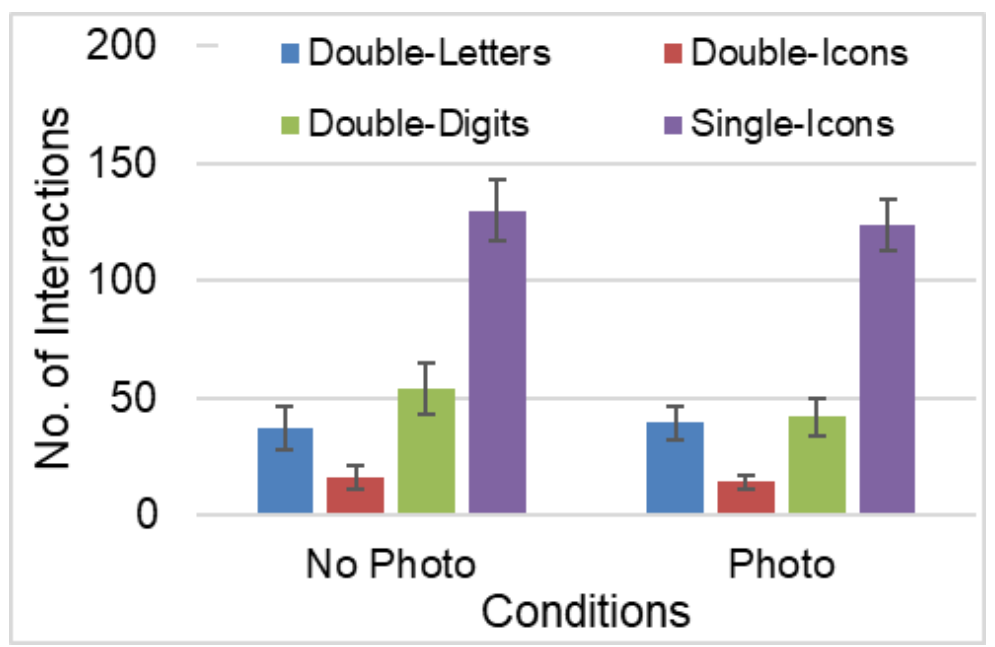

Figure 4.23. Analyses of the number of interactions based on page content.

Again, we analyzed the scrollbar interactions by page content (see Figure 4.23: right). Although the number of interactions was less in pages with photo (54.62/trial, s.d. 130.9) than no photo (59.16/trial, s.d. 121.59), ANOVA did not show any significant effect of content $\left(F_{1,15}=1.94\right.$, $p=.18)$, but significant effect of condition $\left(F_{2.01,30.15}=12.79, p<.001, \eta^{2}=0.18\right)$. There was no content $\times$ condition interaction $\left(F_{1.56,23.4}=0.27, p=.71\right)$.

Post-hoc analysis showed Single-Icons condition had the greatest number of interactions (more than 123.6/trial, s.d. 149.35) among all Landmarked Scrollbar conditions for both photo and no photo cases (all $p<.001$ ). Interestingly, there was no significant difference among the three two columns of landmarks conditions (all $p>.05)$ in any cases.

Summary of Figure 4.22 and Figure 4.23 shows that the presence of an image in the content can reduce mean completion time and the number of interactions during revisitation. We believe the reason for this better performance is the superiority of imagery for forming an association with the landmarks. 


\subsubsection{Analyses of the targets by proximity to landmark}

The targets used in the study were from two groups based on their proximity to the landmarks available on the scrollbar: on a landmark (see Figure 4.24 a and Figure 4.24 c) or near a landmark (see Figure 4.24 b and Figure 4.24 d). We analyzed the performance based on targets' proximity to landmarks.

\section{a.}

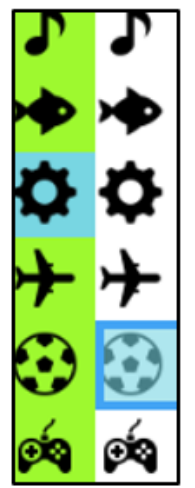

b.

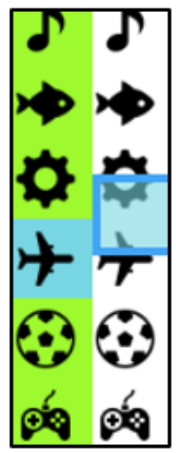

c.

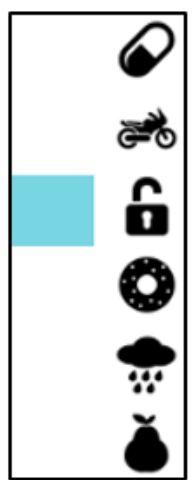

d.

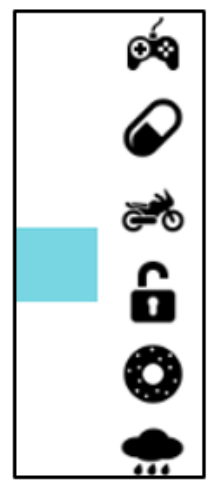

Figure 4.24. On landmark - near landmark in Single-Scrollbar and Double-Scrollbar designs

For trial completion time (see Figure 4.25), ANOVA showed a significant main effect of proximity $\left(F_{1,15}=12.06, p=.003, \eta^{2}=0.03\right)$ and condition $\left(F_{2.16,32.4}=5.61, p=.007, \eta^{2}=0.1\right)$. Also, there was an interaction effect of proximity $\times$ condition $\left(F_{2.49,37.35}=8.56, p<.001, \eta^{2}=0.05\right)$. Post-hoc analysis (Bonferroni-corrected) showed that among near landmark targets Double-Icons was faster (9674ms, s.d. 11310ms) than all other interfaces (all $p<.001$ ), however for on landmark targets the differences were not significant (all $p>.14$ ). 


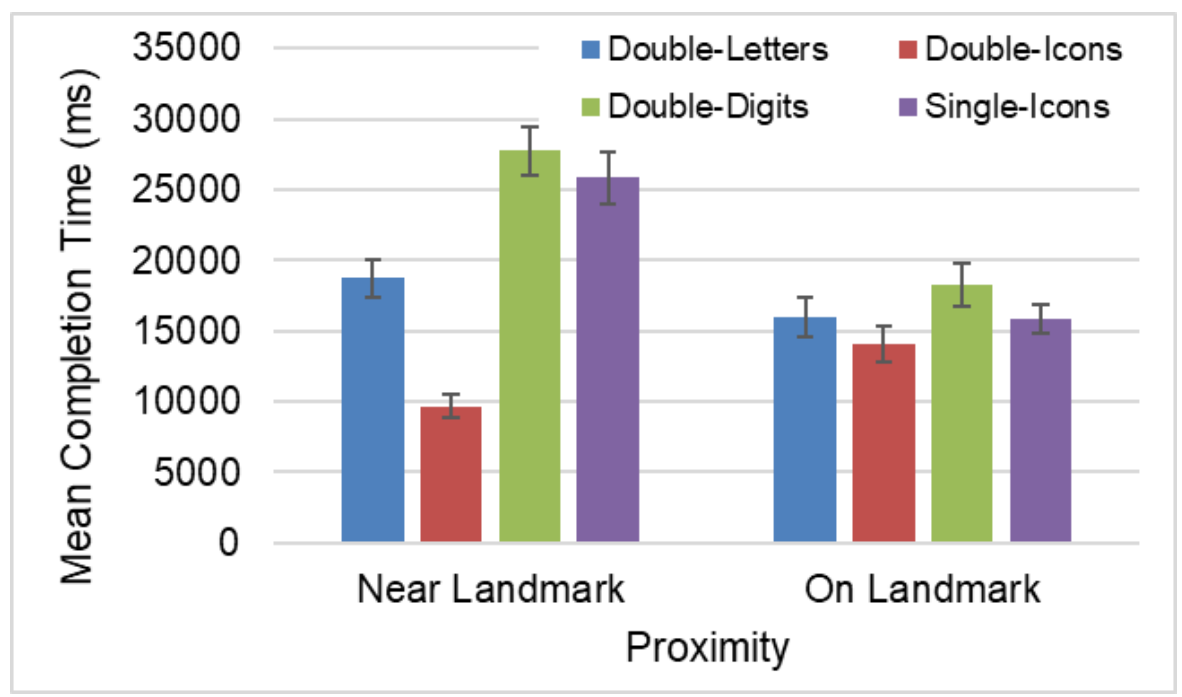

Figure 4.25. Analyses of time by landmark proximity.

We also analyzed the number of interactions made by the participants on the scrollbar based on targets' proximity (see Figure 4.25:right). RM-ANOVA did not show any significant main effect of proximity $\left(F_{1,15}=4.46, p=.05\right)$. But it showed significant main effect of condition $\left(F_{1.92,28.8}=13.98, p<.001, \eta^{2}=0.23\right)$ and proximity $\times$ condition interaction $\left(F_{3,45}=5.71, p=.002\right.$, $\left.\eta^{2}=0.02\right)$.

Post-hoc analysis showed that for on landmark targets, Single-Icons had more than three times the number of scrollbar interactions (104.82/trial, s.d. 158.73) than other conditions (all $p<.001)$. For near landmark targets, Single-Icons again had the most number of interactions (167.34/trial, s.d. 176.93) than other three (all $p<.001$ ). Additionally, Double-Icons had approximately far fewer interactions (10.48/trial, s.d. 37.64) than Double-Digits and Double-Letters (all $p<.02)$. 


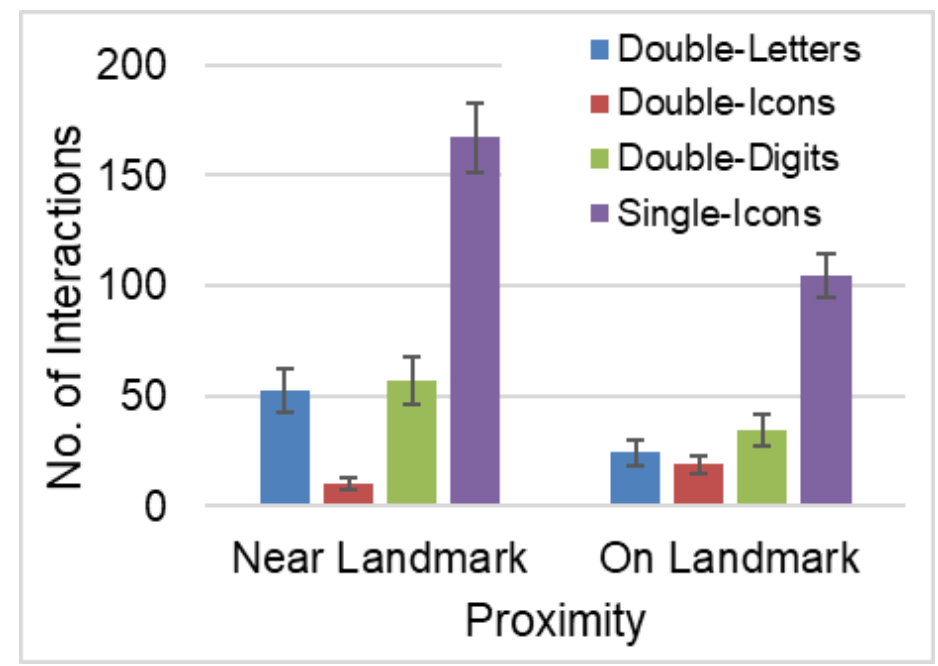

Figure 4.26. Analyses of the number of interactions by landmark proximity.

Our explanation for poorer performance in revisitation for near landmark targets is that in these cases participants need to memorize both the landmark and the amount of distance from those landmarks which increases the cognitive burden for users.

\subsubsection{Fit to the power law of practice}

To examine if there was any learning happening across blocks, we analyzed the fit of users' revisitation time to the power law of practice [59]. Based on this law reaction time for a task decreases with the number of repetitions of that task according to the following formula: $\log \left(T_{n}\right)$ $=\mathrm{C}-\alpha \log (\mathrm{n})$, where $\mathrm{T}_{\mathrm{n}}$ is the time to complete trial $\mathrm{n}, \mathrm{C}$ is the time on the first trial, and $\alpha$ is the steepness of the learning curve. Regression analysis of task completion time across blocks for all landmark augmented scrollbars shows that an almost perfect fit with the power law of practice formula, with $\mathrm{R}^{2}>.85$ for all interfaces (see Figure 4.27). 


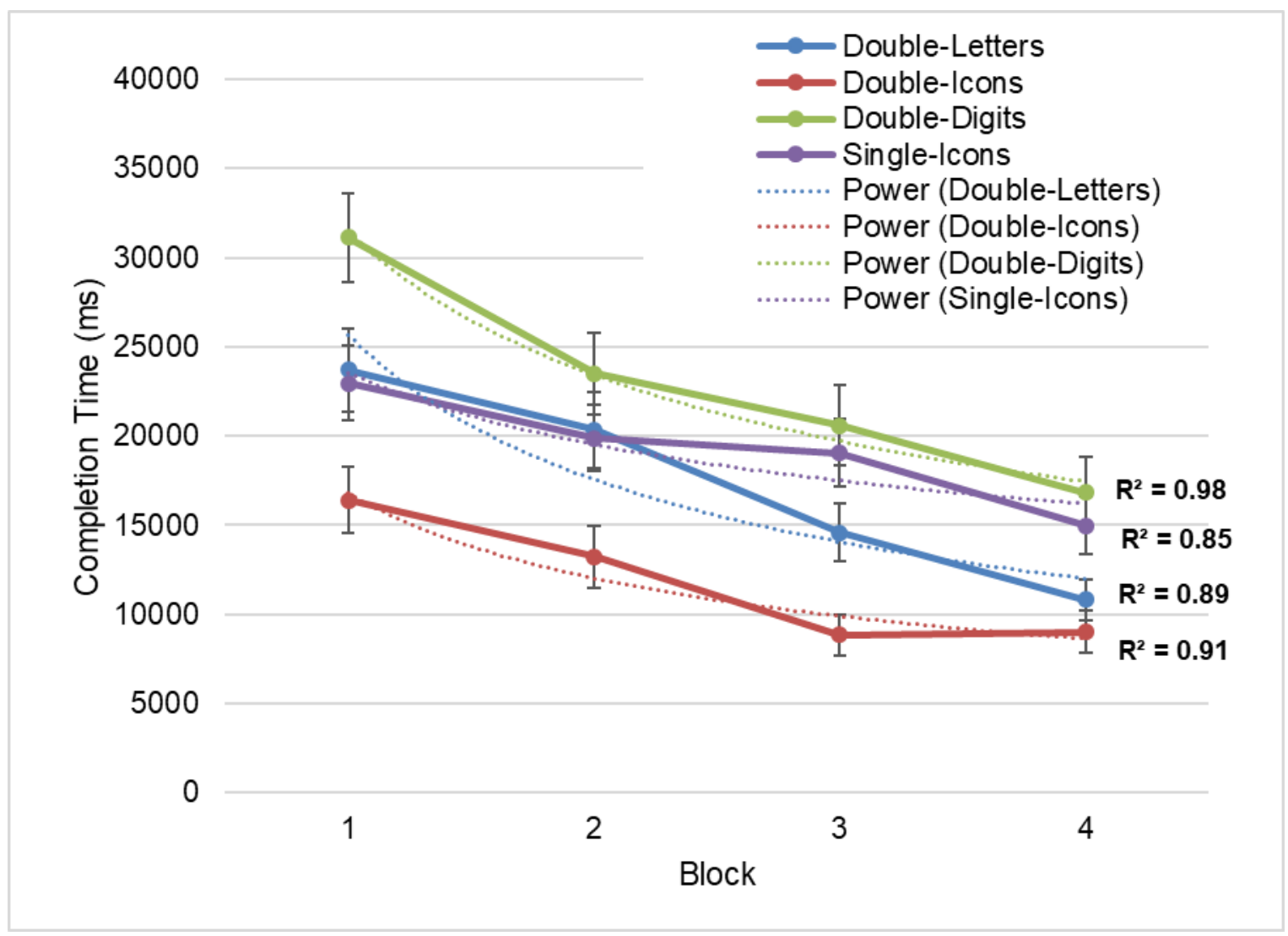

Figure 4.27. Regression analysis of task completion time across blocks for all landmark augmented scrollbars

\subsubsection{Subjective responses}

NASA-TLX responses were also analyzed (using Friedman tests, see Table 4.1). Overall, DoubleIcons condition received lower workload scores and higher performance scores; Double-Digits and Single-Icons conditions received higher workload and lower performance scores. 


\begin{tabular}{|c|c|c|c|c|c|c|}
\hline & $\begin{array}{l}\text { Double } \\
\text { Letters }\end{array}$ & $\begin{array}{c}\text { Double } \\
\text { Icons }\end{array}$ & $\begin{array}{c}\text { Double } \\
\text { Digits }\end{array}$ & $\begin{array}{l}\text { Single } \\
\text { Icons }\end{array}$ & $\chi_{r}^{2}$ & $p$ \\
\hline Mental & $\begin{array}{c}6.31 \\
(2.44)\end{array}$ & $\begin{array}{c}5.0 \\
(2.48)\end{array}$ & $\begin{array}{c}8.5 \\
(1.1)\end{array}$ & $\begin{array}{c}6.62 \\
(1.86)\end{array}$ & 23.76 & .07 \\
\hline Physical & $\begin{array}{c}3.0 \\
(2.28)\end{array}$ & $\begin{array}{c}2.25 \\
(2.14)\end{array}$ & $\begin{array}{c}4.19 \\
(3.17)\end{array}$ & $\begin{array}{l}5.31 \\
(2.5)\end{array}$ & 37.59 & $<.01$ \\
\hline Temporal & $\begin{array}{c}4.25 \\
(2.67)\end{array}$ & $\begin{array}{c}3.19 \\
(1.64)\end{array}$ & $\begin{array}{c}5.56 \\
(2.66)\end{array}$ & $\begin{array}{c}4.81 \\
(2.07)\end{array}$ & 33.08 & $<.01$ \\
\hline Performance & $\begin{array}{c}6.19 \\
(2.37)\end{array}$ & $\begin{array}{c}7.5 \\
(2.1)\end{array}$ & $\begin{array}{c}5.44 \\
(2.45)\end{array}$ & $\begin{array}{c}5.62 \\
(2.03)\end{array}$ & 23.84 & .07 \\
\hline Effort & $\begin{array}{c}5.69 \\
(2.21)\end{array}$ & $\begin{array}{c}4.38 \\
(1.78)\end{array}$ & $\begin{array}{c}7.75 \\
(1.24)\end{array}$ & $\begin{array}{c}6.31 \\
(1.78)\end{array}$ & 23.47 & .07 \\
\hline Frustration & $\begin{array}{c}3.31 \\
(3.24)\end{array}$ & $\begin{array}{c}1.88 \\
(1.71)\end{array}$ & $\begin{array}{c}5.5 \\
(3.01)\end{array}$ & $\begin{array}{c}4.44 \\
(3.01)\end{array}$ & 27.73 & .02 \\
\hline
\end{tabular}

Table 4.1. Mean (s.d.) effort scores (0-10 scale, low to high).

Post-hoc pairwise followup tests showed that Double-Icons was better than Double-Digits in most measures (all $p<.03$ ) except for physical demand and performance. For physical demand and effort requirement measures, Double-Icons received better scores than Single-Icons (all $p<.02$ ).

Participants' ratings of the perceived memorability of the documents using different interfaces (on a 0-10 scale) are shown in Table 4.2. The main clear results from this table are that Double-Icons had more ratings of "Very Easy", and Double-Digits had more ratings of "Hard" and "Very Hard". Preference counts (see Table 4.3) favored both Double-Icons and Double-Letters conditions for all measures; however, Double-Icons was preferred for speed and accuracy measures.

\begin{tabular}{|c|c|c|c|c|}
\hline & $\begin{array}{l}\text { Double } \\
\text { Letters }\end{array}$ & $\begin{array}{c}\text { Double } \\
\text { Icons }\end{array}$ & $\begin{array}{c}\text { Double } \\
\text { Digits }\end{array}$ & $\begin{array}{l}\text { Single } \\
\text { Icons }\end{array}$ \\
\hline Very Easy & 1 & 5 & 0 & 1 \\
\hline Easy & 5 & 4 & 0 & 5 \\
\hline Normal & 4 & 6 & 2 & 3 \\
\hline Hard & 4 & 1 & 10 & 6 \\
\hline Very Hard & 1 & 0 & 4 & 1 \\
\hline
\end{tabular}

Table 4.2. Count of participants rating (0-10) of the ease of memorization for different interfaces. 


\begin{tabular}{|c|c|c|c|c|}
\hline & $\begin{array}{l}\text { Double } \\
\text { Letters }\end{array}$ & $\begin{array}{c}\text { Double } \\
\text { Icons }\end{array}$ & $\begin{array}{c}\text { Double } \\
\text { Digits }\end{array}$ & $\begin{array}{l}\text { Single } \\
\text { Icons }\end{array}$ \\
\hline Speed & 4 & 8 & 0 & 4 \\
\hline Accuracy & 5 & 9 & 1 & 1 \\
\hline Memorization & 6 & 8 & 1 & 1 \\
\hline Comfort & 6 & 7 & 1 & 2 \\
\hline Overall & 6 & 8 & 1 & 1 \\
\hline
\end{tabular}

Table 4.3. Count of participants' preferred interface on five aspects.

\subsubsection{Participants feedback}

Participant comments for the four conditions in general echo the performance results.

Two-level scrollbars provide ease of navigation: the designs expose a tradeoff between having less cognitive load with a single scrollbar and better precision with the Double-Scrollbar designs. Participants appeared to favour the precision of the double-level design. For instance, two participants mentioned ease of navigation with Double-Scrollbar as an advantage: "This type of scrollbar [double scrollbar] felt the most comfortable physically, and it was the easiest to memorize locations within the document. I didn't have to re-click if I was off by a little bit like the one-scrollbar icon system and I rarely needed to scroll with the mouse wheel. " Another participant stated “... because there are two scrollbars I did not have to be as precise in where I clicked with my mouse as I always ended up on the correct page when I clicked the second icon as opposed to the one scrollbar where I always had to scroll quite a bit to get to the correct page since it only got me close." Two other participants complained about difficulty of a precise page selection with one scroll bar: one stated "I had to re-click as I was off by a little bit in the one-scrollbar icon [Single-Icons].”

Presence of mnemonics on the scrollbar assist with revisitation: users' remarks on different interfaces is in accordance with the idea that landmarks can help with the formation of spatial memory. One of the users confirmed that association between content and landmark is easier when icons are present: "[In Double-Icons,] I could make a story using the two icons [to remember the 
page]." Others emphasized on the superiority of icons over digits and letters: "With icons, it didn't take me long to memorize the chapter and pages (and I could remember both without much troubles), so it was way faster.”, “.... think I may have a better visual memory for doing my task daily." And "my photo memory might be better".

Even though icons were the most popular landmark, users' comments showed that some of them preferred letters: "It [letters] gave the most leeway to create my own sentences to remember pages." Also, "I remembered letters better, it made me quicker in using the double scroll bar interface and also I prefer this method over the others."

At the same time, there were varying views on digits as landmarks. Some did not prefer Digits: for example, one participant stated "Digits [in Double-Digits] required high memorization." and another found them difficult to associate to the content of the page, stating "I couldn't match any feature of the document to the numbers on the scrollbar"; but one participant reported them as easier to memorize: "digits were more helpful to memorize". 


\section{CHAPTER 5}

\section{DISCUSSION}

In this chapter, we discuss the findings of our two studies on two-level scrollbar interfaces, present our explanation for each of the findings, and consider how our designs can be used in real-world settings.

\subsection{DISCUSSION OF MAIN FINDINGS}

In the following sections we discuss and explain the three main findings from our two studies: 1) landmarks were useful for revisitation, 2) two columns of landmarks were better than one, and 3) icons were better landmarks than digits or letters.

\subsubsection{All landmarked scrollbars facilitated spatial revisitation}

Our second study revealed that mean trial completion time of all the four artificial landmarksaugmented scrollbars (either single or double) decreased substantially (on average $45.1 \%$ decrease for all interfaces) across blocks (e.g., see Figure 4.17). Also, regarding scrollbar interaction a substantial decrease was noticed ( $25.8 \%$ on average for all blocks) from the first block to the last (e.g., see Figure 4.18). This can be an indication that users were relying more on their spatial memory to return to a previously-visited page simply by rapid recalling of the landmarks available on the scrollbars. Our findings conform to previous knowledge of spatial memory interfaces, which suggests that landmark augmentation can support better spatial memory development $[4,81,82]$.

Our explanation for the above findings is that landmarks on the scrollbar act as reference points in their surrounding environment to help people find locations around them; thus, their absence (in the Standard scrollbar) makes the formation of spatial memory more difficult because there is no landmark nearby. In other words, a lack of artificial landmarks on the scrollbar can hinder a successful revisitation by forcing users to use the nearest available landmarks (e.g., the corners of 
the window) or estimate the absolute location of scrollbar handle (e.g., it is at 5/6 of the scrollbar height) to find their current location in the document. These estimations are most of the time inaccurate and, if the document is long, require many scrollbar interactions to revisit those places in the document later. Significantly higher number of interactions and revisitation time, mentioned at 4.1.3.1 and 4.1.3.2, are an indication that the presence of artificial landmarks on the scrollbar can assist revisitation.

\subsubsection{Two columns of landmarks are better than one}

A previous lab study [82] showed that a single-column landmarked scrollbar can be useful in shorter documents, but with a 900-page document, each individual landmark in the single column covers 30 pages, which slow down the formation of spatial memory and rapid revisitation of target pages. In this case, even if users recall the right landmark, it takes them many mouse scroll interactions to find the right page. Our main study's results, especially concerning the number of interactions (see Figure 4.14 and Figure 4.18), indicate that having two columns of landmarks in the interface improves the revisitation performance in a long document, compared to a single column of landmarks. In this regard, Single-Icon required significantly more time, and significantly more scrollbar interaction to find a target page compared to Double-Icon. For example, in case of the last block of the experiment, people required 10 interactions to navigate to the right page using Double-Icon compared to 113 interactions for Single-Icon.

We believe that the lack of fine-grained navigation in the Single-Icon design is the reason for the high number of scrollbar interactions. It is interesting to note that users were most accurate (counting only their first click) with the Single-Icon design (see Figure 4.21), likely because the presence of landmarks on the scrollbar helped them find the approximate location of the page in the document. Yet revisitations were difficult since a slight change of the scrollbar handle, even a few millimeters, moves participants several pages away from the desired location. Therefore, they had to then resort to scrolling in order to locate the target, thus increasing the number of interactions (Figure 4.14 and Figure 4.18) and increasing physical demand (see Table 4.1), which was also reflected in users comments: "... as opposed to the one scrollbar where I always had to scroll quite a bit to get to the correct page since it only got me close." Having two columns of 
landmarks in the scrollbar enables users to revisit pages more precisely by providing a fine-grained navigation.

Furthermore, since we did not compare Double-Scrollbar augmented with landmark with plain Double-Scrollbar, we cannot directly claim that the presence of landmarks in Double-Scrollbar led to a better revisitation experience. One possible scenario can be that just the fine-grained navigation that was offered by Double-Scrollbar was enough to assist revisitation, and landmark augmentation did not make any difference.

However, by comparing performance of users using three different versions of Double-Scrollbar with icons, letters and digits, we can infer that presence of landmarks had a positive impact on the formation of spatial memory. As the study results suggest, using icons was the most effective type of landmark augmentation, and using digits was the worst, with letters being in between. This difference in performance suggests that if users were solely relying on fine-grained navigational feature of Double-Scrollbar and ignored the landmarks, then performance for all three mentioned interfaces would have been similar.

\subsubsection{Icons perform better as landmarks than letters and digits}

Completion time results, number of interactions, workload measures, and participant preferences all favored icons over letter and digit landmarks. Double-Digits, as our second study suggests, required $94 \%$ more time and $210 \%$ more interactions (e.g., for the last block Double-Digit on average required $16892 \mathrm{~ms}$ and 35 interactions compared to Double-Icon with $8994 \mathrm{~ms}$ and 10 interactions). Furthermore, $87 \%$ of users considered memorization of the document with DoubleDigits to be "Hard" or "Very hard", while only 6.2\% of users gave these ratings for the DoubleIcon scrollbar. Several factors may have led to these results. First, even though the icons used in Double-Icons condition were abstract (i.e., there was no connection to page content), they were highly distinctive in shape. Each conveyed a distinct meaning that enabled users to form connections (even made-up ones) with page contents. In some cases, connections that users created were somewhat odd (e.g., if a page was marked by two very different icons), but people were still able to form associations. This conforms to what is already known about memory techniques such 
as the 'method of loci' $[67,87,92]$ that allow the linking of abstract concepts with concrete spatial objects to support stronger association and thus better recollection.

As users' comments showed, forming association by telling odd stories was one of the techniques participants used to make the consolidation of memory easier. For instance, one user mentioned: "I could make a story using the two icons [to remember the page]." In fact, previous studies indicates that story telling helps participants recall items faster and with higher accuracy. For example, Bower et. al. asked participants to memorize 12 lists of ten words using either a traditional rehearsal method or a story-based method. The group that used narratives recalled the lists of words six to seven times better than the other group [13]. Given the superiority of stories in the formation of memory, we can imagine why using icons as landmarks was more effective in creating a better revisitation experience than letters and digits.

Letters and digits lack the same degree of meaningful features (and digits more so), making it more difficult for users to connect them with the contents of the page. Users' comments also pointed this difficulty out: "I couldn't match any feature of the document to the numbers on the scrollbar"; another user reported, "It's hard to build connection between the digits and the text." As a result, though letters and digits were able to support spatial learning (i.e., they were spatially stable and differentiable), users had more difficulty forming the needed associations.

The second reason is the way our brain is developed to work better with images than words and digits. As discussed in section 2.6.1, pictures are easier to recognize, memorize, and recall than words (the "picture superiority effect"), which has been found in many studies $[22,40,75,85]$.

Third, the contents of the long document used in the experiment were random in order (although pages were grouped by topic). The letters and digits were placed in ascending order, and this may have conflicted with the existing use of letters in alphabetically-sorted documents [3]. This can be an interesting area to explore further, as previous work showed the advantages of sorted letters for revisiting phonebook words [3].

Fourth, (and similarly) the two columns of digits available in the Double-Digits scrollbar may have conflicted with the idea of page numbers, which is also prevalent in most documents. The digit 
landmarks did not have any connection to the actual page numbers, and this may have caused difficulties for some users. To revisit a page in the Double-Icons scrollbar, users needed to recall a chunked four-digit number like '11-29' (two digits from the left and two from the right scrollbar), in a way we remember PINs for ATM. Although chunking is an efficient way to remember numbers [37,55], memorizing several locations, each with their unique code, proved to be challenging (e.g., reflected in Study 2's time-out results, see Figure 4.20). As a result, participants preferred scrolling rather than memory-based clicking on the scrollbar.

\subsection{GENERALIZATION OF FINDINGS}

Even though we ran our experiments in a lab setting and used a customized document viewer with a limited number of participants, we believe our results can be generalized to real world situations. Our quantitative and qualitative results indicate that the two-level scrollbar can be feasibly employed in commercial software to address the problem of revisitation in long documents. Although the Double-Scrollbar would not be the ultimate solution to revisitation problems in all situations, and although there will be areas where it would not be as effective as other revisitation methods (such as small-screen devices), it can be a simple and effective tool in the designers' toolbox in many cases.

The Double-Scrollbar design is intuitive. According to intuitive design principles, an interaction method is intuitive if its users can employ the system without having to use their reasoning and mental processing, or having to maintain a high cognitive load [10,52]. Our analyses show that people learned how to work with the Double-Scrollbar within a short time (through a practice round). Then, in the experiment, they were asked to perform a few revisitation tasks. In many cases, users were faster and more accurate working with the Double-Scrollbar, than the conventional Single-Scrollbar as the system they have been working with for many years. Results obtained from our workload questionnaire and preference counts, also, showed that the DoubleIcons design required less temporal demand and provided more comfort. Moreover, an intuitive design works by relying on users' previous knowledge without breaking users mental model. Since 
the Double-Scrollbar design is based on multiple-granularity selection, which is prevalent in some common UI elements, such as date pickers, it is familiar to users, thus it meets many of the criteria for an intuitive design.

Since the Double-Scrollbar design does not require a significant change in the previous interface design paradigms, it can be easily integrated into most of the commercial software. Technically, it is easy to implement, and once implemented, it does not require high processing power or storage space, which makes it a useful tool to be added to previous document viewers to address revisitation problem in long videos.

Implementation of this interaction method is straightforward. For a web-based application, the designer should hide the existing scrollbar, add the two-level scrollbar to the interface, and map it with the length of the document. Then, based on the size and resolution of the screen, the designer should augment both scrollbars with landmarks. Coding all these functions in HTML, CSS, and jQuery should not take more than 200 lines of code, and once it is coded, it can be easily added to existing web-based frameworks, such as Vue.js and jQuery UI. Cross-platform capability of web tools guarantees the availability of this design to a wide range of devices. The implementation would be similar for desktop applications as well; the only challenge for desktop application development is that support for the Double-Scrollbar design would have to be added to GUI Software Development Kits.

Uddin, et al. [82] explored the idea of augmented slider with a video player and showed that it would be a good solution for revisitation in non-text content. Therefore, we can assume that the Double-Scrollbar design augmented with landmarks should works well with other types of content that is different from the textual material used in our study (e.g., video and audio content and type of content that is provided in a predefined symbolic form such as music notes or genome sequence). 


\subsection{USE CASE EXAMPLES}

Here we provide some use case examples of Double-Scrollbar design in other settings. These use cases are intended to explore the Double-Scrollbar's applicability with other content, meaning it is not limited to text viewers only, but it can be employed anywhere revisitation in long linear sequences is needed.

\subsubsection{Genome browser}

People working in the fields of bioinformatics and genomics use genome browsers to analyze, annotate, and visualize the long sequence of DNA data both in isolation and in collaborative settings. Figure 5.1 shows a mock-up of a genome browser with two rows of artificial landmarks on it (laid out horizontally). Locations of interest on the chromosome can be mapped to the landmarks, and clicking on a pair of landmarks will enable users to navigate to a selected location (zoomed at the bottom). Later, those locations can be revisited easily by remembering the landmarks. 


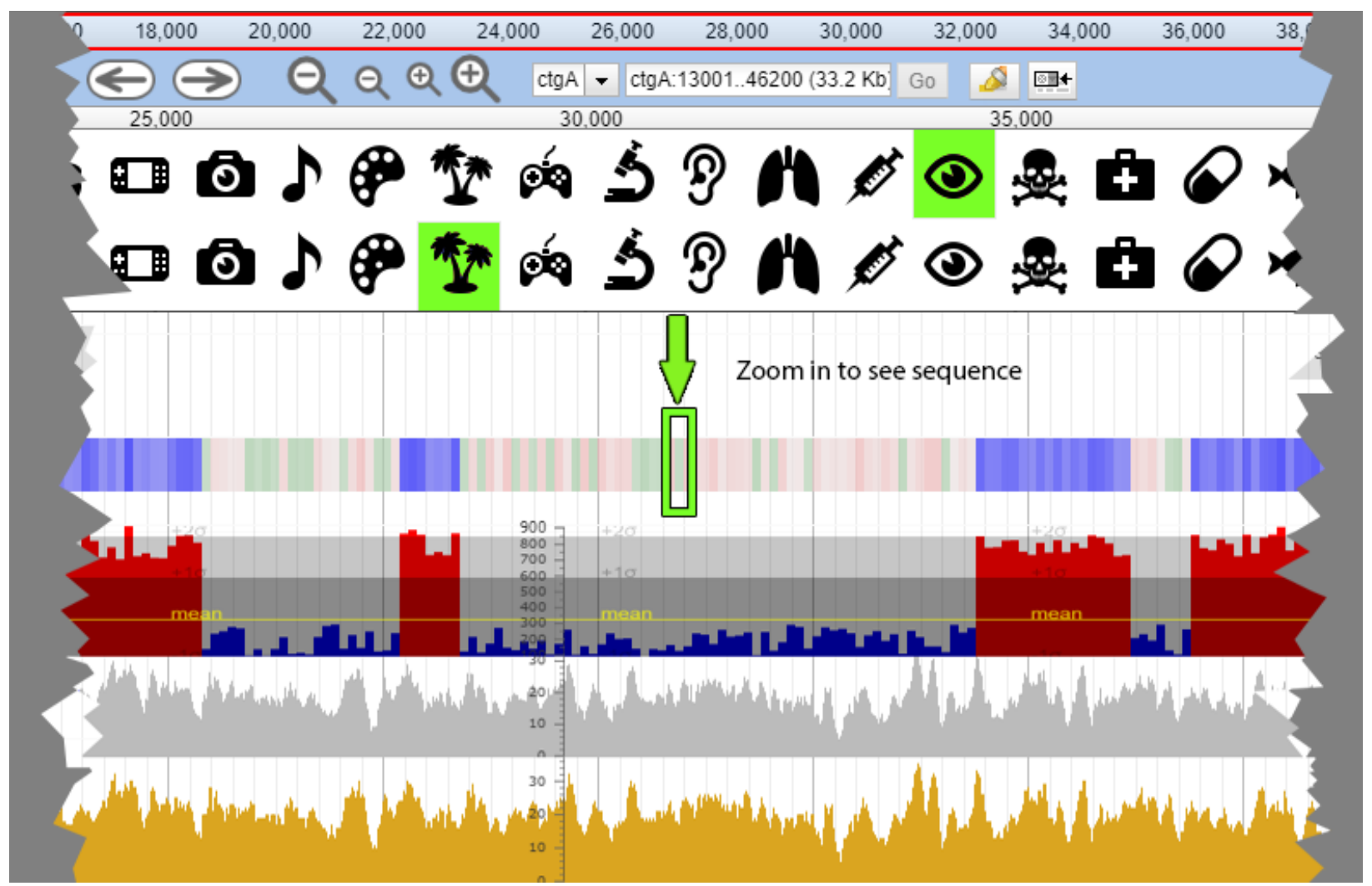

Figure 5.1. Mock up genome browser

\subsubsection{Video player}

Videos cannot easily be navigated using a regular search method, which can make content revisitation a challenge. Current commercial systems usually offer a small, low resolution preview of the content to enable revisitation. However, since these previews lack details in many cases, they fail to serve as reliable revisitation tools. Moreover, previews, due to space constraints are a very small sample of the whole video ${ }^{3}$. For example, Figure $\mathbf{5 . 2}$ shows a ten-frame preview of a 20-minute programming tutorial on YouTube [93] (one preview frame for every two minutes).

\footnotetext{
${ }^{3}$ there are at least 36,000 frames in a 20 minutes video with $30 \mathrm{fps}$
} 
Due to size constraints, the previews are hard to distinguish, thus it is very difficult to rely on these previews to find a desirable part of the content.
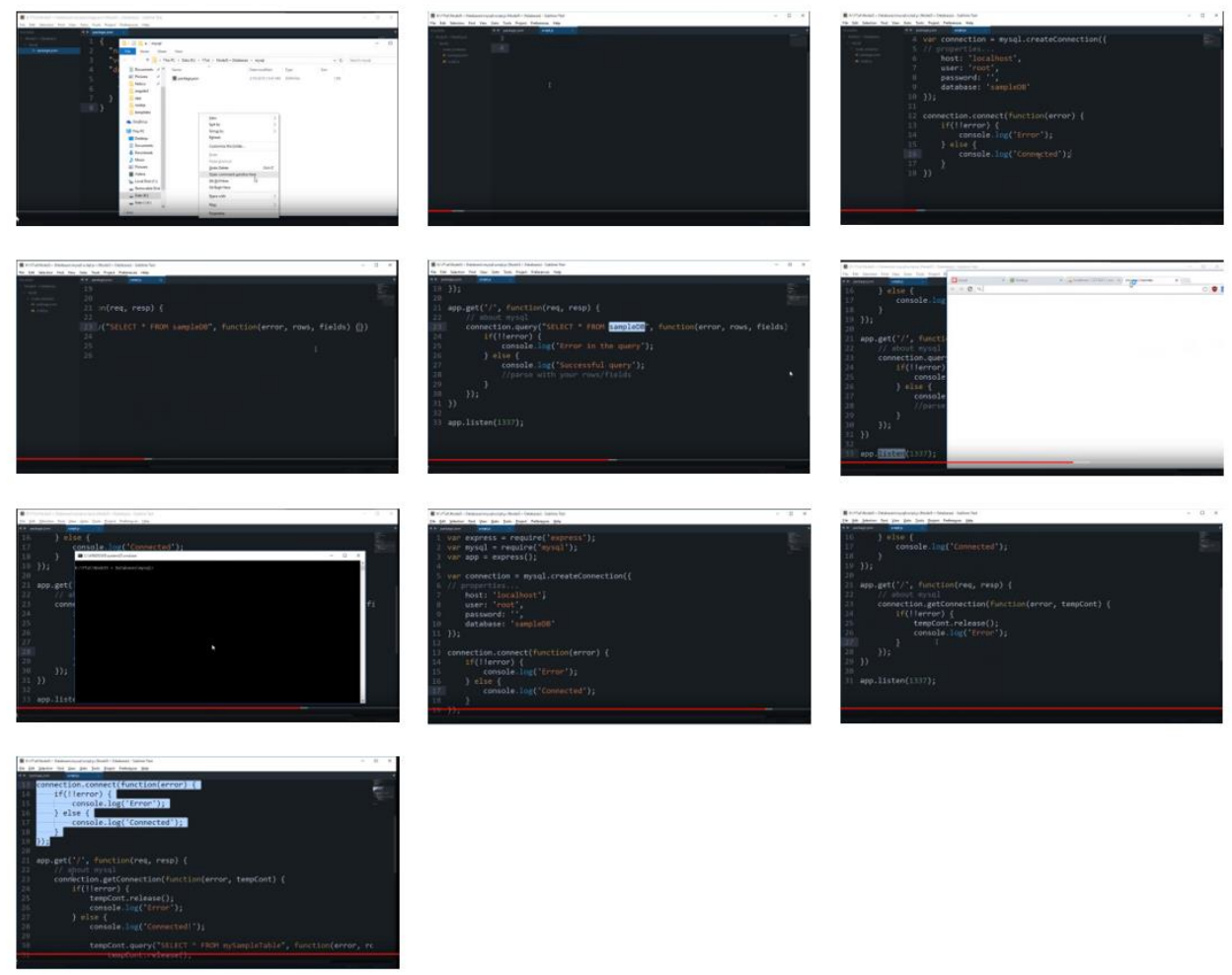

Figure 5.2. Previews of a twenty-minutes video on YouTube timeline

In a previous study, Uddin, et al. explored the idea of augmenting timeline of a video player with one level of icons and showed that their suggested design would help with revisitation in a short video (132 seconds)[82]. However, when the video is long, a one-level icon-augmented timeline does not provide enough granularity in selection. Figure $\mathbf{5 . 3}$ shows a mock-up of a video player with two levels of icon landmarks. This situation would also apply to audio players, which have even more problems with providing landmarks, as unlike video players there is no preview available and users must solely rely on the timeline. 


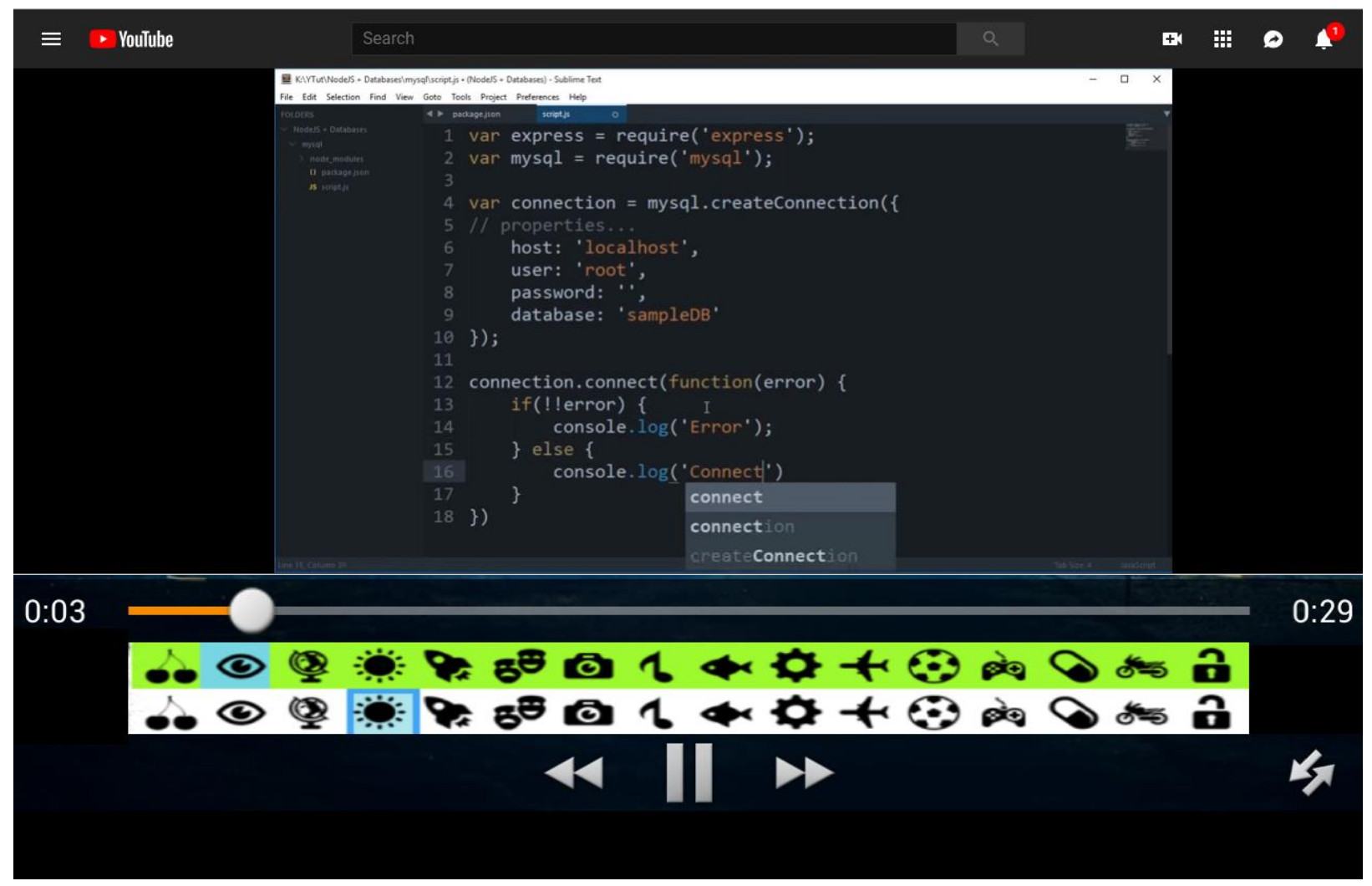

Figure 5.3. Mock up video player with two levels of scrollbar augmented with icons 


\section{CHAPTER 6}

\section{CONCLUSION}

Although linear navigation controls in document viewers provide a spatial representation of a document and allow effective navigation and revisitation, they become inefficient in long documents. We investigated the use of artificial landmarks to improve revisitation in long documents - inspired by visual enhancements seen in books (e.g., indent cuts or page-edge marks). We augmented a scroll-bar style controller for a linear document viewer with different artificial landmarks-including icons, letters, and digits, in one and two columns. We tested these landmark-augmentations in studies where participants visited and revisited multiple locations in very long documents. All augmentation techniques improved spatial learning and revisitation performance. Also, using two columns of landmarks resulted in the best performance, lowest effort scores, and highest user preferences. Our studies showed that artificial landmarks would be promising in forming strong spatial memory in long documents, and would substantially improve revisitation performance.

\subsection{CONTRIBUTIONS}

Below, three main contributions of this thesis are presented:

\subsubsection{All Landmark-augmented scrollbars helped with revisitation}

The main study showed that revisitation time for all four augmented scrollbars (single or double) decreased substantially across blocks, following a power law of learning [59]. This can be an indication that people were using spatial memory to visit a previously-visited page simply by recalling the landmarks. Our findings conform to previous knowledge of spatial memory 
interfaces, which had suggested that landmark-augmentation can support spatial memory development.

\subsubsection{The use of two columns of landmarks is feasible}

Figure 4.13 and Figure 4.18 show that participants were more successful in revisiting pages when two columns of landmarks were present. Both studies showed that two columns of icon landmarks improved revisitation performance compared to a single column of icons - both in terms of time and number of interactions. Having two columns of landmarks in the scrollbar enabled users to revisit pages more precisely (fewer interactions) by connecting two landmarks to the page of interest.

\subsubsection{The Double-Icon design provided the best performance}

Completion time results, number of interactions, workload measures, and participant preferences were all in favor of icons over letter and digit landmarks. We believe icons have greater potential to convey a distinct meaning, enabling users to form connections with the content of their desired pages and form stronger spatial memories. In contrast, letters and digits did not assist in making useful associations.

\subsection{FUTURE WORK}

\subsubsection{Different document content}

The two studies showed that using two columns of landmarks can improve document revisitation in situations where one column of landmarks is too coarse. We believe two-column landmark scrollbars could be integrated easily into existing interaction paradigms to enhance revisitation experience for non-text documents, such as long video clips, podcasts, and data types that are represented as symbols (e.g., genomic data). As a result, examining how people make the association with landmark when the document is not text, can be a new direction to be explored. 


\subsubsection{Auto extracted icons}

In both studies presented in this thesis, we used random icons with no relation to the content of the page they were mapped to. However, investigating the impact of icons' relevance to content can be another direction for future investigation. Even though we could not find any research specifically on the topic of the impact of landmark relevance to the formation of spatial memory, we believe there are two areas of interest.

One body of research indicates that story telling helps participants recall items faster and with higher accuracy (e.g. Bower's study showed that a narrative-based memorization strategy led to much better recall [13]). Given the superiority of relevant stories in the formation of memory, we are interested in whether icons that match the content can be recalled faster and with higher precision.

On the other hand, there are other studies indicating that odd stories are more memorable. For instance, one study asked participants to recognize two group of photos that had been shown to them two days before. Photos in the first group were images of random objects such as animals, cars, people, etc. The second group comprised of photos which were striking and out of the ordinary (for example, a dog holding a pipe). Participants were able to distinguish $66 \%$ of photos in the first group and $88 \%$ of photos in the second group, suggesting that odd stories can help with the formation of strong memories [77]. Consequently, we can imagine the presence of random icons on the scrollbar force users to make stories that are odd or out-of-context, which could lead to more efficient revisitation.

\subsubsection{Small-screen devices}

An interesting area of future research is the use of multi-level scrollbars in small devices. First, the view port in handheld devices is smaller which may make it difficult to include a large number of landmarks. Moreover, unlike desktop and laptop devices which enable pixel-level accuracy of selection, the "fat finger problem" reduces selection accuracy on small-screen devices. This is a problem for revisitation in handheld devices because navigation to a part of a document using a 
scrollbar require a precise movement of scrollbar handle, which is very difficult if not impossible when using a fingertip to work with touch screens.

There are other challenges in augmenting scrollbars with landmarks in small screens. For instance, due to space constraints, scrollbars in most small screens are hidden until the user starts to navigate in the document. The hidden scrollbar could hamper the formation of spatial memory as the underlying assumption in augmenting a scrollbar with landmarks is that users can form spatial memory as they use the document (i.e., they can see the scrollbar all the time). Another challenge, also due to the size of small devices, is that we cannot have as many landmarks on the scrollbar, which limits the number of pages that can be selected precisely using the two-level scrollbar.

\subsubsection{More than two levels of scrollbars}

It will also be interesting to examine the efficiency of a triple-scrollbar design, which might require more mental effort but offers more precise navigation. This design could be beneficial in different areas, such as small-screen devices where the height of the device reduces the number of icons in each column, or in very long documents (e.g., a genome sequence).

One of our major concerns about the two-level scrollbar is that it would force the memorization of two sets of icons that would lead to the increase of the mental work load and distraction from the main goal of revisitation. However, our study suggested that people are willing to trade a little more mental load for a faster and more accurate navigation which is offered in the two-scrollbar design.

In the area of Geocoding (converting address to coordinates), a company called what3words [94] has divided the map of the earth into a grid of $3 \mathrm{~m} \times 3 \mathrm{~m}$ squares and assigned each a unique combination of three words (e.g., "tests.doctors.eggs" for the Thorvaldson building at the University of Saskatchewan). They claim that their system eliminates the effort of dealing with lengthy numbers by replacing latitude and longitudes with simple words, thus, decreasing ambiguity and increasing memorability of locations. 
Users' positive experience in working with hierarchical menus can be another indication that users might be able to work reasonably well with the triple-scrollbar design. Hierarchical menus organize content in different levels to provide ease of information retrieval when the arrangement of information is unstructured. However, Miller, et. al. reported that the accuracy and speed in target acquisition in the hierarchical menus can be highly dependent on breadth (number of icons in each level) and depth (number of levels) of the menu [54]. In his experiment, Miller investigated the difference in users' performance when working with four varieties of menu depth and breath. He reported that an increase in depth can negatively impact users' short-term memory by increasing information overload. On the other hand, an increase in breadth increases accuracy but at the same time leads to screen clutter, and is not probably a good solution for the small screen.

\subsubsection{Combination with other techniques}

The idea of landmark-augmented scrollbars can also be used with previous methods to increase their effectiveness. For instance, visualizing bookmarks and "Read Wear" on the scrollbar proved to be effective in some cases. Laakso's bookmarking [45] and Alexander's Footprints [4] both have the advantage of representing read-wear or bookmarks on the scrollbar. This design allows for an immediate access to the revisitation marks, which seems to be an efficient way of representing them in terms of speed, the number of errors, and user preference [4], although these techniques are more effective with fewer number of bookmarks.

In a scrollbar-based bookmarking system, similar-looking squares are used to represent the revisitation marks (see Figure 6.1 A), and these marks do not possess enough features to distinguish them from the rest the revisitation marks. Due to this lack of distinctiveness, once there are several revisitation marks present, people need to remember which revisitation mark was 
mapped to which part of the content. Rapid and effective revisitation depends on people easily recalling which revisitation mark is mapped to which part of the content. Therefore, exploring landmark-augmented revisitation marks can be a direction to enhance revisitation experience even further and thus an interesting opportunity for research.

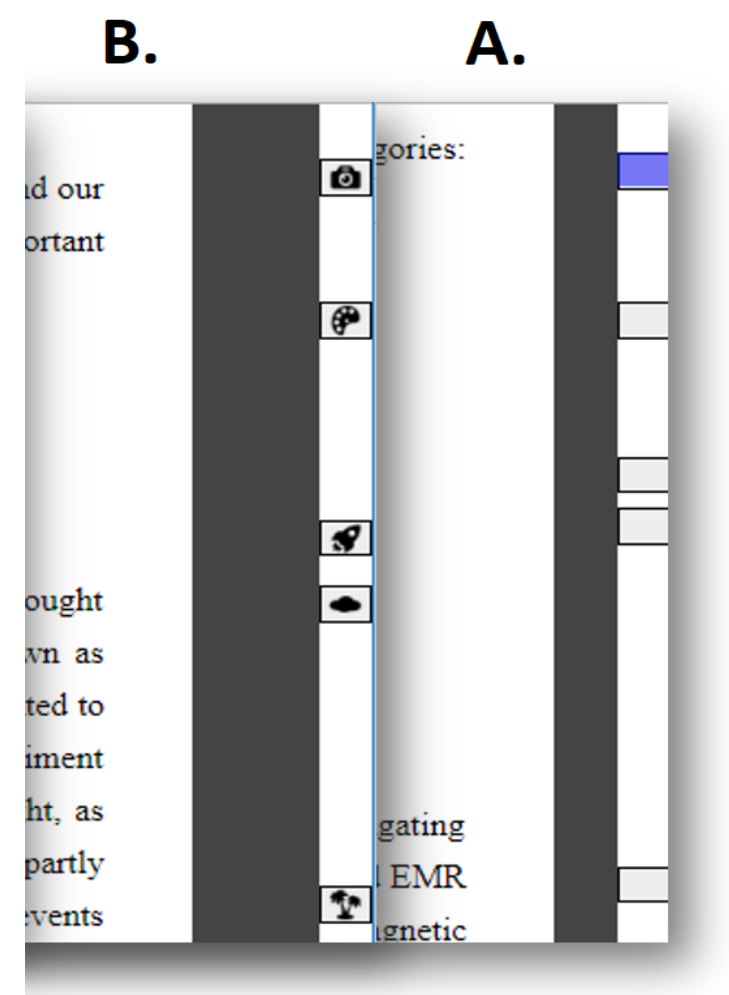

Figure 6.1. A: Unadorned bookmarks on the scrollbar. B: Icon-augmented bookmarks on the scrollbar

In conclusion, we showed that linear navigation controls in document viewers do not provide sufficient support for the formation of spatial memory; therefore, in long documents their effectiveness for revisitation is questionable. We explored the idea of the Double-Scrollbar augmented with artificial landmarks to help both the formation of spatial memory and precise navigation of long documents. We compared icons, letters, and digits as three types of common landmarks, and reported on the effectiveness of each one. Our results show that all landmark- 
augmentation techniques improved spatial learning, and that two levels of icon landmarks improved revisitation performance significantly compared to other interfaces. 


\section{REFERENCES}

1. David Abrams, Ron Baecker, and Mark Chignell. 1998. Information archiving with bookmarks. In Proceedings of the SIGCHI conference on Human factors in computing systems - CHI '98, 41-48. https://doi.org/10.1145/274644.274651

2. Eytan Adar, Jaime Teevan, and Susan T. T. Dumais. 2008. Large scale analysis of web revisitation patterns. In Proceeding of the twenty-sixth annual CHI conference on Human factors in computing systems - CHI '08, 1197. https://doi.org/10.1145/1357054.1357241

3. Christopher Ahlberg and Ben Shneiderman. 1994. The alphaslider. In Proceedings of the SIGCHI conference on Human factors in computing systems celebrating interdependence CHI '94, 365-371. https://doi.org/10.1145/191666.191790

4. Jason Alexander, Andy Cockburn, Stephen Fitchett, Carl Gutwin, and Saul Greenberg. 2009. Revisiting read wear: analysis, design, and evaluation of a footprints scrollbar. In Proceedings of the 27th international conference on Human factors in computing systems CHI '09, 1665-1674. https://doi.org/10.1145/1518701.1518957

5. Farzaneh Khabbazibab Anari, Mansoureh Sajjadi, and Firooz Sadighi. 2014. The Magic of Mnemonics for Vocabulary Learning of a Second Language. http://www.sciencepublishinggroup.com $\quad 3, \quad 1: \quad 1$. https://doi.org/10.11648/J.IJLL.S.2015030101.11

6. Richard C. Atkinson. 1975. Mnemotechnics in second-language learning. American Psychologist 30, 8: 821-828. https://doi.org/10.1037/h0077029

7. Richard C. Atkinson and Michael R. Raugh. 1975. An application of the mnemonic keyword method to the acquisition of a Russian vocabulary. Journal of Experimental Psychology: Human Learning and Memory 1, 2: 126-133. https://doi.org/10.1037/02787393.1.2.126

8. Anne Aula, Natalie Jhaveri, and Mika Käki. 2005. Information search and re-access strategies of experienced web users. In Proceedings of the 14th international conference on World Wide Web - WWW '05, 583. https://doi.org/10.1145/1060745.1060831

9. Gilles Bailly, Eric Lecolinet, and Laurence Nigay. 2008. Flower menus. In Proceedings of the working conference on Advanced visual interfaces - AVI '08, 15. https://doi.org/10.1145/1385569.1385575

10. Jorn Blackler, Alethea Liane \& Hurtienne. 2007. Towards a unified view of intuitive interaction : definitions, models and tools across the world. Inst. für Arbeitswissenschaft, 
RWTH Aachen. Retrieved October 18, 2018 from http://eprints.qut.edu.au/19116/

11. Daniel Bor. 2012. The ravenous brain : how the new science of consciousness explains our insatiable search for meaning. Basic Books.

12. Daniel Bor, John Duncan, Richard J Wiseman, and Adrian M Owen. 2003. Encoding strategies dissociate prefrontal activity from working memory demand. Neuron 37, 2: 3617. Retrieved October 8, 2018 from http://www.ncbi.nlm.nih.gov/pubmed/12546829

13. Gordon H. Bower and Michal C. Clark. 1969. Narrative stories as mediators for serial learning. Psychonomic Science 14, 4: 181-182. https://doi.org/10.3758/BF03332778

14. Stuart K. Card, Jock D. Mackinlay, and Ben. Shneiderman. 1999. Readings in information visualization : using vision to think. Morgan Kaufmann Publishers. Retrieved October 5, 2018 from https://dl.acm.org/citation.cfm?id=300769

15. Marvin M. Chun and Yuhong Jiang. 1998. Contextual Cueing: Implicit Learning and Memory of Visual Context Guides Spatial Attention. Cognitive Psychology 36, 1: 28-71. https://doi.org/10.1006/COGP.1998.0681

16. Andy Cockburn, Andy Cockburn, Saul Greenberg, and Saul Greenberg. 1999. Getting Back to Back: Alternate Behaviors for a Web Browser's Back Button. Retrieved November 12, 2018 from http://citeseerx.ist.psu.edu/viewdoc/summary?doi=10.1.1.46.5899

17. Andy Cockburn, Carl Gutwin, and Jason Alexander. 2006. Faster document navigation with space-filling thumbnails. In Proceedings of the SIGCHI conference on Human Factors in computing systems - CHI '06, 1. https://doi.org/10.1145/1124772.1124774

18. ANDY COCKBURN and BRUCE MCKENZIE. 2001. What do web users do? An empirical analysis of web use. International Journal of Human-Computer Studies 54, 6: 903-922. https://doi.org/10.1006/IJHC.2001.0459

19. Jacob Cohen. 1973. Eta-squared and partial eta-squared in communication science. Human Communication Research 28, 56: 473-490.

20. Nelson Cowan, Zhijian Chen, and Jeffrey N. Rouder. 2004. Constant Capacity in an Immediate Serial-Recall Task. Psychological Science 15, 9: 634-640. https://doi.org/10.1111/j.0956-7976.2004.00732.x

21. Nelson Cowan, Jeffrey N Rouder, Christopher L Blume, and J Scott Saults. 2012. Models of verbal working memory capacity: what does it take to make them work? Psychological review 119, 3: 480-99. https://doi.org/10.1037/a0027791

22. Tim Curran and Jeanne Doyle. 2011. Picture Superiority Doubly Dissociates the ERP Correlates of Recollection and Familiarity. Journal of Cognitive Neuroscience 23, 5: 12471262. https://doi.org/10.1162/jocn.2010.21464 
23. R. DeLine, M. Czerwinski, B. Meyers, G. Venolia, S. Drucker, and G. Robertson. 2006. Code Thumbnails: Using Spatial Memory to Navigate Source Code. In Visual Languages and Human-Centric Computing (VL/HCC'06), 11-18. https://doi.org/10.1109/VLHCC.2006.14

24. Alain Desrochers, Collette Gélinas, and Linda D. Wieland. 1989. An application of the mnemonic keyword method to the acquisition of German nouns and their grammatical gender. Journal of Educational Psychology 81, 1: 25-32. https://doi.org/10.1037/00220663.81.1.25

25. Trien V. Do and Roy A. Ruddle. 2017. MyWebSteps: Aiding Revisiting with a Visual Web History. Interacting with Computers 29, 4: 530-551. https://doi.org/10.1093/iwc/iww038

26. Steven N. Durlauf and Lawrence E. Blume. 2008. The New Palgrave: Dictionary of Economics. Palgrave Macmillan UK, London. https://doi.org/10.1007/978-1-349-58802-2

27. K A Ericcson, W G Chase, and S Faloon. 1980. Acquisition of a memory skill. Science (New York, N.Y.) 208, 4448: 1181-2. Retrieved October 8, 2018 from http://www.ncbi.nlm.nih.gov/pubmed/7375930

28. K A Ericsson and W Kintsch. 1995. Long-term working memory. Psychological review 102, 2: 211-45. Retrieved October 8, 2018 from http://www.ncbi.nlm.nih.gov/pubmed/7740089

29. Mariano García, Albert N Badre, and John T Stasko. 1994. Development and validation of icons varying in their abstractness. Interacting with Computers 6, 2: 191-211. https://doi.org/10.1016/0953-5438(94)90024-8

30. Emmanouil Giannisakis, Gilles Bailly, Sylvain Malacria, and Fanny Chevalier. 2017. IconHK. In Proceedings of the 2017 CHI Conference on Human Factors in Computing Systems - CHI '17, 4715-4726. https://doi.org/10.1145/3025453.3025595

31. David Gittins. 1986. Icon-based human-computer interaction. International Journal of Man-Machine Studies 24, 6: 519-543. https://doi.org/10.1016/S0020-7373(86)80007-4

32. Saul Greenberg. 1993. The computer user as toolsmith : the use, reuse, and organization of computer-based tools. Cambridge University Press.

33. Carl Gutwin and Andy Cockburn. 2006. Improving list revisitation with ListMaps. In Proceedings of the working conference on Advanced visual interfaces - AVI '06, 396. https://doi.org/10.1145/1133265.1133347

34. Carl Gutwin, Andy Cockburn, Joey Scarr, Sylvain Malacria, Scott C. Olson, Carl Gutwin, Andy Cockburn, Joey Scarr, Sylvain Malacria, and Scott C. Olson. 2014. Faster command selection on tablets with FastTap. In Proceedings of the 32nd annual ACM conference on Human factors in computing systems - CHI '14, 2617-2626. 
https://doi.org/10.1145/2556288.2557136

35. EL Mohamad Haj, Roy Kessels, Laurent Urso, and Jean Louis Nandrino. 2018. Chunking to improve verbal forward spans in Korsakoff's syndrome. Applied Neuropsychology: Adult: 1-8. https://doi.org/10.1080/23279095.2018.1499023

36. Sandra G. Hart and Lowell E. Staveland. 1988. Development of NASA-TLX (Task Load Index): Results of Empirical and Theoretical Research. Advances in Psychology 52: 139183. https://doi.org/10.1016/S0166-4115(08)62386-9

37. Kenneth L. Higbee. 2001. Your memory: how it works and how to improve it. Marlowe \& Co.

38. William C. Hill, James D. Hollan, Dave Wroblewski, and Tim McCandless. 1992. Edit wear and read wear. In Proceedings of the SIGCHI conference on Human factors in computing systems - CHI '92, 3-9. https://doi.org/10.1145/142750.142751

39. Graham J. Hitch, Neil Burgess, John N. Towse, and Vicki Culpin. 1996. Temporal Grouping Effects in Immediate Recall: A Working Memory Analysis. The Quarterly Journal of Experimental Psychology Section A 49, 1: 116-139. https://doi.org/10.1080/713755609

40. W. E. HOCKLEY. 2008. The picture superiority effect in associative recognition. Memory \& Cognition 36, 7: 1351-1359. https://doi.org/10.3758/MC.36.7.1351

41. Shamsi T. Iqbal and Eric Horvitz. 2007. Disruption and recovery of computing tasks. In Proceedings of the SIGCHI conference on Human factors in computing systems - CHI '07, 677. https://doi.org/10.1145/1240624.1240730

42. Shaun Kaasten and Saul Greenberg. 2001. Integrating back, history and bookmarks in web browsers. In CHI '01 extended abstracts on Human factors in computing systems - CHI '01,379. https://doi.org/10.1145/634067.634291

43. Zeynab Khalafi and Mohammad Reza Oroji. 2016. The Impact of Using Keyword Method on Vocabulary Learning and Retention: A Case of Iranian Intermediate EFL Learners. International Journal of Language and Linguistics 4, 1: 9. https://doi.org/10.11648/j.ijl1.20160401.12

44. Gordon Kurtenbach and William Buxton. 1994. User learning and performance with marking menus. In Proceedings of the SIGCHI conference on Human factors in computing systems celebrating interdependence - CHI '94, 258-264. https://doi.org/10.1145/191666.191759

45. Sari A. Laakso, Karri Pekka Laakso, and Asko J. Saura. 2000. Improved scroll bars. In CHI '00 extended abstracts on Human factors in computing systems - CHI '00, 97. https://doi.org/10.1145/633292.633350 
46. Lee and Allison. 1992. Investigations into history tools for user support. Retrieved October 9, 2018 from https://dl.acm.org/citation.cfm?id=171663

47. Kevin Lynch. 1960. The image of the city. MIT Press.

48. Eleanor A. Maguire, Richard S. J. Frackowiak, and Christopher D. Frith. 1997. Recalling Routes around London: Activation of the Right Hippocampus in Taxi Drivers. The Journal of Neuroscience 17, 18: 7103-7110. https://doi.org/10.1523/JNEUROSCI.17-1807103.1997

49. Justin Matejka, Tovi Grossman, and George Fitzmaurice. 2013. Swifter. In Proceedings of the SIGCHI Conference on Human Factors in Computing Systems - CHI '13, 1159. https://doi.org/10.1145/2470654.2466149

50. Matthias Mayer. 2007. Web History Tools and Revisitation Support: A Survey of Existing Approaches and Directions. Foundations and Trends ${ }^{\circledR}$ in Human-Computer Interaction 2, 3: 173-278. https://doi.org/10.1561/1100000011

51. Mark A. McDaniel and Michael Pressley (eds.). 1987. Imagery and Related Mnemonic Processes. Springer New York, New York, NY. https://doi.org/10.1007/978-1-4612-46763

52. Everett N. McKay. 2013. UI is communication: how to design intuitive, user centered interfaces by focusing on effective communication. Elsevier, Morgan Kaufmann.

53. Md. Sami Uddin. 2016. Improving Multi-Touch Interactions Using Hands as Landmarks. University of Saskatchewan. Retrieved September 7, 2017 from http://hci.usask.ca/publications/view.php?id=401

54. Dwight P. Miller. 1981. The Depth/Breadth Tradeoff in Hierarchical Computer Menus. Proceedings of the Human Factors Society Annual Meeting 25, 1: 296-300. https://doi.org/10.1177/107118138102500179

55. George A. Miller. 1956. The magical number seven, plus or minus two: some limits on our capacity for processing information. Psychological Review 63, 2: 81-97. https://doi.org/10.1037/h0043158

56. Dan Morris, Meredith Ringel Morris, and Gina Venolia. 2008. SearchBar. In Proceeding of the twenty-sixth annual CHI conference on Human factors in computing systems - CHI'08, 1207. https://doi.org/10.1145/1357054.1357242

57. Richard Morris. 1984. Developments of a water-maze procedure for studying spatial learning in the rat. Journal of Neuroscience Methods 11, 1: 47-60. https://doi.org/10.1016/0165-0270(84)90007-4

58. PAUL MUTER and CANDACE MAYSON. 1986. The role of graphics in item selection 
from menus. Behaviour \& Information Technology 5, 1: 89-95. https://doi.org/10.1080/01449298608914502

59. Allen Newell and Paul S. Rosenbloom. 1981. Mechanisms of skill acquisition and the law of practice. Cognitive skills and their acquisition 1: 1-55.

60. Dennis Graham Norris and Kristjan Kalm. 2018. Chunking and redintegration in verbal short-term memory. https://doi.org/10.31234/OSF.IO/SM736

61. Dennis Graham Norris and Kristjan Kalm. 2018. What's in a chunk? Chunking and data compression in verbal short-term memory. https://doi.org/10.31234/OSF.IO/2ST4J

62. Hartmut Obendorf, Harald Weinreich, Eelco Herder, and Matthias Mayer. 2007. Web page revisitation revisited. In Proceedings of the SIGCHI conference on Human factors in computing systems - CHI '07, 597. https://doi.org/10.1145/1240624.1240719

63. M. Osada, Holmes Liao, and B. Shneiderman. 1993. Alpha Slider: Searching Textual Lists with Sliders. Retrieved October 6, 2018 from https://drum.lib.umd.edu/handle/1903/5397

64. Yi Pan, Yafeng \& Hu. 2018. Psychology of Education. Retrieved October 8, 2018 from https://www.researchgate.net/publication/324797461_Mnemonic_techniques_and_trainin $\mathrm{g}$

65. P. Pappachan and M. Ziefle. 2008. Cultural influences on the comprehensibility of icons in mobile-computer interaction. Behaviour \& Information Technology 27, 4: 331-337. https://doi.org/10.1080/01449290802228399

66. Simon T. Perrault, Eric Lecolinet, Yoann Pascal Bourse, Shengdong Zhao, and Yves Guiard. 2015. Physical Loci. In Proceedings of the 33rd Annual ACM Conference on Human Factors in Computing Systems - CHI '15, 299-308. https://doi.org/10.1145/2702123.2702126

67. Simon T. Perrault, Eric Lecolinet, Yoann Pascal Bourse, Shengdong Zhao, and Yves Guiard. 2015. Physical Loci: leveraging spatial, object and semantic memory for command selection. In Proceedings of the 33rd Annual ACM Conference on Human Factors in Computing Systems - CHI '15, 299-308. https://doi.org/10.1145/2702123.2702126

68. Albert Postma and Edward H.F. De Haan. 1996. What Was Where? Memory for Object Locations. The Quarterly Journal of Experimental Psychology A 49, 1: 178-199. https://doi.org/10.1080/027249896392856

69. Kai-Florian Richter and Stephan Winter. 2014. Introduction: What Landmarks Are, and Why They Are Important. In Landmarks. Springer International Publishing, Cham, 1-25. https://doi.org/10.1007/978-3-319-05732-3_1

70. George Robertson, Mary Czerwinski, Kevin Larson, Daniel C. Robbins, David Thiel, and 
Maarten van Dantzich. 1998. Data mountain. In Proceedings of the 11th annual ACM symposium on User interface software and technology - UIST '98, 153-162. https://doi.org/10.1145/288392.288596

71. T.L. Saaty and M.S. Ozdemir. 2003. Why the magic number seven plus or minus two. Mathematical and Computer Modelling 38, 3-4: 233-244. https://doi.org/10.1016/S08957177(03)90083-5

72. Joey Scarr, Andy Cockburn, Carl Gutwin, and Andrea Bunt. 2012. Improving command selection with CommandMaps. In Proceedings of the 2012 ACM annual conference on Human Factors in Computing Systems - CHI '12, 257. https://doi.org/10.1145/2207676.2207713

73. Katherine Schramm, Carl Gutwin, and Andy Cockburn. 2016. Supporting Transitions to Expertise in Hidden Toolbars. In Proceedings of the 2016 CHI Conference on Human Factors in Computing Systems - CHI '16, 4687-4698. https://doi.org/10.1145/2858036.2858412

74. Sabine Schröder and Martina Ziefle. 2008. Effects of Icon Concreteness and Complexity on Semantic Transparency: Younger vs. Older Users. In Computers Helping People with Special Needs. Springer Berlin Heidelberg, Berlin, Heidelberg, 90-97. https://doi.org/10.1007/978-3-540-70540-6_12

75. Roger N. Shepard. 1967. Recognition memory for words, sentences, and pictures. Journal of Verbal Learning and Verbal Behavior 6, 1: 156-163. https://doi.org/10.1016/S00225371(67)80067-7

76. Amy Skopik and Carl Gutwin. 2005. Improving revisitation in fisheye views with visit wear. In Proceedings of the SIGCHI conference on Human factors in computing systems CHI '05, 771. https://doi.org/10.1145/1054972.1055079

77. Lionel Standing. 1973. Learning 10000 pictures. Quarterly Journal of Experimental Psychology 25, 2: 207-222. https://doi.org/10.1080/14640747308400340

78. J. A. Szirmai. 1999. The archaeology of medieval bookbinding. Ashgate. Retrieved October 4, 2018 from https://books.google.ca/books/about/The_archaeology_of_medieval_bookbinding.html?id =VgxUAAAAMAAJ\&redir_esc=y

79. Linda Tauscher and Saul Greenberg. 1997. Revisitation patterns in World Wide Web navigation. In Proceedings of the SIGCHI conference on Human factors in computing systems - CHI '97, 399-406. https://doi.org/10.1145/258549.258816

80. Md. Sami Uddin and Carl Gutwin. 2016. Rapid Command Selection on Multi-Touch Tablets with Single-Handed HandMark Menus. In Proceedings of the 2016 ACM on Interactive Surfaces and Spaces - ISS '16, 205-214. 
https://doi.org/10.1145/2992154.2992172

81. Md. Sami Uddin, Carl Gutwin, and Andy Cockburn. 2017. The Effects of Artificial Landmarks on Learning and Performance in Spatial-Memory Interfaces. In Proceedings of the 2017 CHI Conference on Human Factors in Computing Systems - CHI '17, 3843-3855. https://doi.org/10.1145/3025453.3025497

82. Md. Sami Uddin, Carl Gutwin, and Alix Goguey. 2017. Using Artificial Landmarks to Improve Revisitation Performance and Spatial Learning in Linear Control Widgets ACM Reference format. In Proceedings of ACM symposium on Spatial User Interaction - SUI 2017, In press. https://doi.org/10.1145/3131277.3132184

83. Md. Sami Uddin, Carl Gutwin, and Benjamin Lafreniere. 2016. HandMark Menus: Rapid Command Selection and Large Command Sets on Multi-Touch Displays. In Proceedings of the 2016 CHI Conference on Human Factors in Computing Systems - CHI '16, 58365848. https://doi.org/10.1145/2858036.2858211

84. James Wen and James Wen. 2003. Post-Valued Recall web pages: User disorientation hits the big time. IT \& SOCIETY 1: 184--194. Retrieved November 12, 2018 from http://citeseerx.ist.psu.edu/viewdoc/summary?doi=10.1.1.3.7688

85. Andrew J. O. Whitehouse, Murray T. Maybery, and Kevin Durkin. 2006. The development of the picture-superiority effect. British Journal of Developmental Psychology 24, 4: 767773. https://doi.org/10.1348/026151005X74153

86. Susan Wiedenbeck. 1999. The use of icons and labels in an end user application program: An empirical study of learning and retention. Behaviour \& Information Technology 18, 2: 68-82. https://doi.org/10.1080/014492999119129

87. Frances A. Yates. 1966. The art of memory. University of Chicago Press, Chicago.

88. Chun Yu, Ravin Balakrishnan, Ken Hinckley, Tomer Moscovich, and Yuanchun Shi. 2013. Implicit bookmarking: Improving support for revisitation in within-document reading tasks. International Journal of Human-Computer Studies 71, 3: 303-320. https://doi.org/10.1016/J.IJHCS.2012.10.012

89. Jian Zhao, Daniel Wigdor, and Ravin Balakrishnan. 2013. TrailMap. In Proceedings of the SIGCHI Conference on Human Factors in Computing Systems - CHI '13, 3009. https://doi.org/10.1145/2470654.2481417

90. landmark | Definition of landmark in English by Oxford Dictionaries. Retrieved November 14, 2018 from https://en.oxforddictionaries.com/definition/landmark

91. Visual Studio Tips and Tricks: Enhanced Scroll Bar - Go DevMENTAL. Retrieved November $\quad 24, \quad 2018$ from https://blogs.msdn.microsoft.com/cdnstudents/2014/01/21/visual-studio-tips-and-tricks- 
enhanced-scroll-bar/

92. Method of loci.

93. NodeJS + MySQL Database Connection Tutorial - YouTube. Retrieved October 20, 2018 from https://www.youtube.com/watch?v=hGZX_SA71Yg

94. what3words | Addressing the world. Retrieved February 7, 2019 from https://what3words.com/

95. 1997. How people revisit web pages: empirical findings and implications for the design of history systems. International Journal of Human-Computer Studies 47, 1: 97-137. https://doi.org/10.1006/IJHC.1997.0125 


\section{APPENDIX}

\section{DEPARTMENT OF COMPUTER SCIENCE UNIVERSITY OF SASKATCHEWAN INFORMED CONSENT FORM}

- UNIVERSITY OF

Research Project:

Double Scrollbar Study - Summer 2017

Investigators:

Dr. Carl Gutwin, Professor, Department of Computer Science (966-8646)

Md. Sami Uddin and Ehsan Sotoodeh Mollashahi, Grad Student, Department of Computer Science

This consent form, a copy of which has been given to you, is only part of the process of informed consent. It should give you the basic idea of what the research is about and what your participation will involve. If you would like more detail about something mentioned here, or information not included here, please ask. Please take the time to read this form carefully and to understand any accompanying information.

This study is concerned with evaluating the performance and learnability of new kind of landmark-based document viewer.

The goal of the research is to evaluate the performance and learnability of revisitation in document viewer.

The session will require about 60 minutes, during which you will be asked to revisit specific location of the document using the specified interfaces shown in a desktop system in the Human-Computer Interaction Lab at the University of Saskatchewan.

At the end of the session, you will be given more information about the purpose and goals of the study, and there will be time for you to ask questions about the research. As a way of thanking you for your participation and to help compensate you for your time and any travel costs you may have incurred, you will receive a $\mathbf{\$ 1 0}$ honorarium at the end of the session.

The data collected from this study will be used in articles for publication in journals and conference proceedings.

As one way of thanking you for your time, we will be pleased to make available to you a summary of the results of this study once they have been compiled (usually within two months). This summary will outline the research and discuss our findings and recommendations. This summary will be available on the HCI lab's website: http://www.hci.usask.ca/

All personal and identifying data will be kept confidential. Confidentiality will be preserved by using pseudonyms in any presentation of textual data in journals or at conferences. The informed consent form and all research data will be kept in a secure location under confidentiality in accordance with University policy for 5 years post publication. Do you have any questions about this aspect of the study?

You are free to withdraw from the study at any time without penalty and without losing any advertised benefits. Withdrawal from the study will not affect your academic status or your access to services at the university. If you withdraw, your data will be deleted from the study and destroyed. Your right to withdraw data from the study will apply until results have been disseminated, data has been pooled, etc. After this, it is possible that some form of research dissemination will have already occurred and it may not be possible to withdraw your data.

Your continued participation should be as informed as your initial consent, so you should feel free to ask for clarification or new information throughout your participation. If you have further questions concerning matters related to this research, please contact:

- Dr. Carl Gutwin, Professor, Dept. of Computer Science, (306) 966-8646, gutwin@es.usask.ca

Your signature on this form indicates that you have understood to your satisfaction the information regarding participation in the research project and agree to participate as a participant. In no way does this waive your legal rights nor release the investigators, sponsors, or involved institutions from their legal and professional responsibilities. If you have further questions about this study or your rights as a participant, please contact:

- Dr. Carl Gutwin, Professor, Dept. of Computer Science, (306) 966-8646, gutwin@cs.usask.ca

- Research Ethics Office, University of Saskatchewan, (306) 966-2975 or toll free at 888-966-2975.

Participant's signature:

Date:

Investigator's signature:

Date:

A copy of this consent form has been given to you to keep for your records and reference. This research has the ethical approval of the Research Ethics Office at the University of Saskatchewan.

\section{Figure A.1 Consent form}




\section{Study Questionnaire}

Please evaluate the task you just completed by carefully selecting the value on the scale from 0 to 10 at the point which matches your experience.

NOTE: Performance is measured on a scale where 0 is Poor and 10 is Good.

* Required

\section{Participant ID *}

Please ask the experimenter if not provided.

Your answer

Interface Type *

What type of landmark was available in the scrollbar of the interface? [Ask the experimenter if you are not sure]

Choose

\section{Mental Demand *}

How much mental and perceptual activity was required (e.g., thinking, deciding, calculating. remembering, looking, searching, etc.) to perform the task? Was the task easy or demanding. simple or complex, forgiving or exacting?

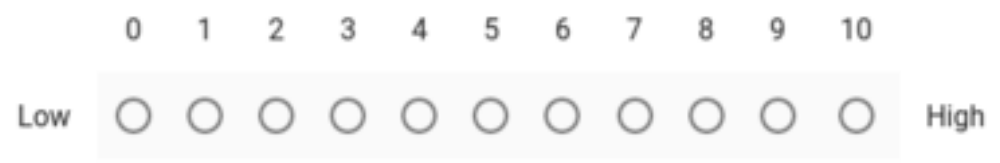

\section{Physical Demand *}

How much physical activity was required (e.g. pressing, finger movement, controlling, activating. etc.) to perform the task? Was the task easy or demanding, slow or brisk, slack or strenuous, restful or laborious?
$0 \quad 1$
34
$\begin{array}{lll}5 & 6 & 7\end{array}$
$8 \quad 9 \quad 10$
Low
000
$\circ \bigcirc \bigcirc \bigcirc \bigcirc$
High

Figure A.2 Study questionnaire part 1 


\section{Temporal Demand *}

How much time pressure did you feel due to the rate at which the task elements occurred? Was the pace slow and leisurely or rapid and frantic?

\section{$\begin{array}{lllllllllll}0 & 1 & 2 & 3 & 4 & 5 & 6 & 7 & 8 & 9 & 10\end{array}$ \\ Low $\mathrm{O} O \mathrm{O} O \mathrm{O} O \mathrm{O} O \mathrm{O}$ High}

\section{Performance *}

How successful do you think you were in accomplishing the goals of the task set by the experimenter? How satisfied were you with your performance in accomplishing these goals?

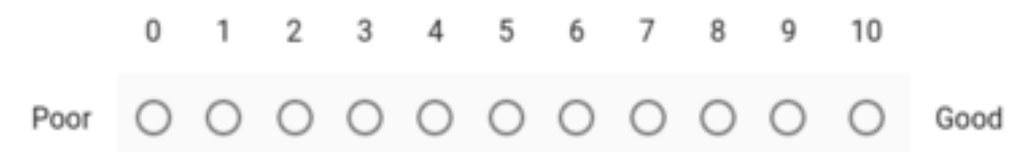

\section{Effort *}

How hard did you have to work (mentally and physically) to accomplish your level of performance?

$$
\begin{array}{lllllllllllll} 
& 0 & 1 & 2 & 3 & 4 & 5 & 6 & 7 & 8 & 9 & 10 & \\
\text { Low } \bigcirc & \bigcirc & \bigcirc & \bigcirc & \bigcirc & \bigcirc & \bigcirc & \bigcirc & \bigcirc & \bigcirc & \bigcirc & \text { High }
\end{array}
$$

\section{Frustration *}

How insecure, discouraged, irritated, stressed and annoyed versus secure, gratified, content, relaxed and complacent did you feel during the task?

$$
\begin{array}{lllllllllllll} 
& 0 & 1 & 2 & 3 & 4 & 5 & 6 & 7 & 8 & 9 & 10 & \\
\text { Low } \bigcirc & \bigcirc & \bigcirc & \bigcirc & \bigcirc & \bigcirc & \bigcirc & \bigcirc & \bigcirc & \bigcirc & \bigcirc & \text { High }
\end{array}
$$

\section{Explanation *}

How did you remember the locations of the Pdf document? Did any feature of the Pdf Viewer/Scrollbar help you to revisit the document locations? Please explain how (or why those were not helpful).

Your answer

Figure A.3 Study questionnaire part 2 


\section{Preference}

Based on you experience of using four systems, please answer the following questions carefully.

\section{Easiness *}

How easy were the contents to remember?

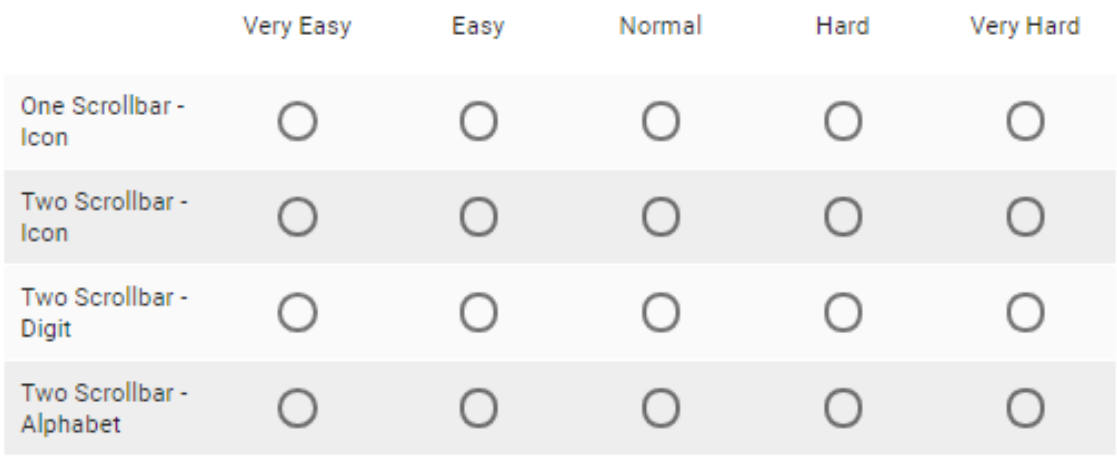

\section{Preferences *}

Between the four systems which one would you prefer based on your performance for the following categories?

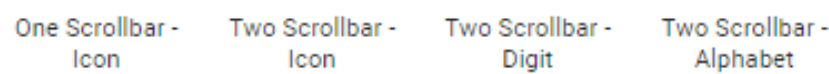

\begin{tabular}{|c|}
\hline Speed \\
\hline Accuracy \\
\hline Memoriz \\
\hline Comfort \\
\hline Overall \\
\hline
\end{tabular}

\section{Reason *}

Among the five categories of the last question (speed, accuracy, memorization, comfort and overall), what was/were the reason/s for your preference? Please explain briefly.

Figure A.4 Study questionnaire part 3 


\title{
Study Questionnaire - Overall
}

\author{
Please fill out the following questions carefully. \\ * Required \\ Participant ID * \\ Ask the experimenter if not provided \\ Your answer \\ Age * \\ In years \\ Your answer
}

Sex *

Select one.

Male

Female

\section{Document Viewers *}

What document viewer(s)/application(s) do you use regularly to view (pdf) documents ? E.g. Adobe Acrobat reader, Foxit reader, etc.

Your answer

\section{Usage *}

How much time do you spend on using Document Viewer in a week (e.g. Adobe Acrobat reader, Foxit reader, etc.)?

0 Hours

$1 \cdot 10$ Hours

(11-20 Hours

$20+$ Hours

Figure A.5 Demographic questionnaire 